\title{
Bulletin of \\ The Natural History \\ Museum
}
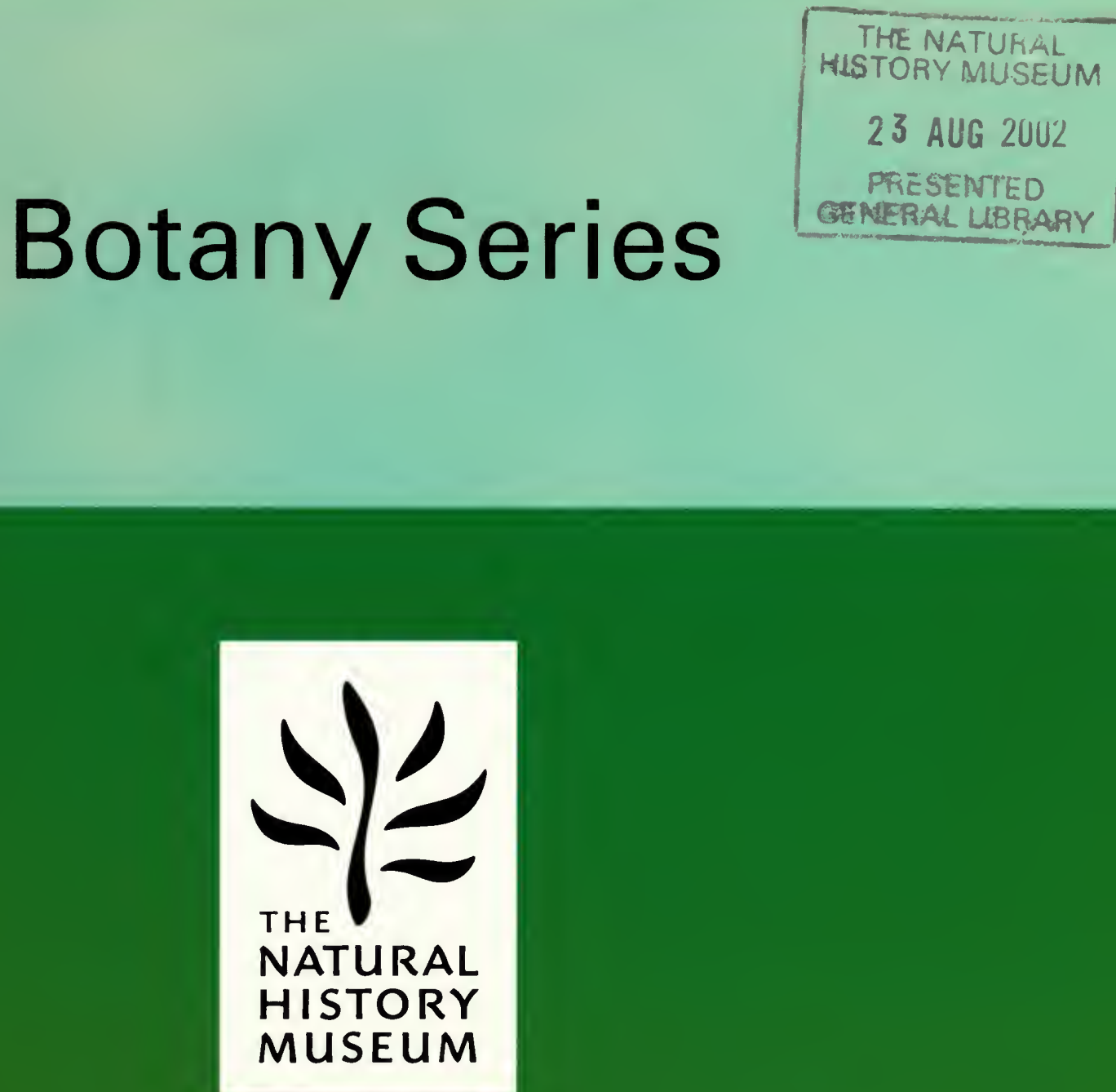


\section{The Bulletin of The Natural History Museum (formerly: Bulletin of the British Museum (Natural History) ), instituted in 1949, is issued in four scientific series, Botany, Entomology, Geology (incorporating Mineralogy) and Zoology.}

The Botany Series is edited in the Museum's Department of Botany

Keeper of Botany:

Prof. R. Bateman

Editor of Bulletin:

Ms S.A. Henderson

Papers in the Bulletin are primarily the results of research carried out on the unique and ever-growing collections of the Museum, both by the scientific staff and by specialists from elsewhere who make use of the Museum's resources. Many of the papers are works of reference that will remain indispensable for years to come. All papers submitted for publication are subjected to external peer review before acceptance.

SUBSCRIPTIONS Bulletin of the Natural History Museum, Botany Series (ISSN 0968-0446) is published twice a year (one volume per annum) in June and November. Volume 32 will appear in 2002. The 2002 subscription price (excluding VAT) of a volume, which includes print and electronic access, is $£ 88.00$ (US \$155.00 in USA, Canada and Mexico). The electronic-only price available to institutional subscribers is $£ 79.00$ (US $\$ 140.00$ in USA, Canada and Mexico).

ORDERS Orders, which must be accompanied by payment, may be sent to any bookseller, subscription agent or direct to the publisher: Cambridge University Press, The Edinburgh Building, Shaftesbury Road, Cambridge CB2 2RU, UK; or in the USA, Canada and Mexico: Cambridge University Press, Journals Department, 40 West 20th Street, New York, NY 1011-4211, USA. EU subscribers (outside the UK) who are not registered for VAT should add VAT at their country's rate. VAT registered members should provide their VAT registration number. Japanese prices for institutions (including ASP delivery) are available from Kinokuniya Company Ltd, P.O. Box 55, Chitose, Tokyo 156, Japan. Prices include delivery by air. Postmaster: send address changes in USA, Canada and Mexico to: Bulletin of the Natural History Museum, Botany Series, Cambridge University Press, 110 Midland Avenue, Port Chester, New York, NY 105783-4930. Claims for missing issues should be made immediately on receipt of the subsequent issues.

Orders and enquiries for issues prior to 2002 should be sent to: Intercept Ltd., P.O. Box 716, Andover, Hampshire SP10 1YG, Telephone: (01264) 334748, Fax: (01264) 334058, Email: intercept@andover.co.uk, Internet: http://www.intercept.co.uk

ADVERTISING Apply to the Publisher. Address enquiries to the Advertising Promoter.

COPYRIGHT AND PERMISSIONS This journal is registered with the Copyright Clearance Center, 222 Rosewood Drive, Danvers, MA 01923, USA. Organizations in the USA who are also registered with CCC may therefore photocopy material (beyond the limits permitted by Section 107 and 108 of US Copyright law) subject to payment to CCC of the per-copy fee of $\$ 16.00$. This consent does not extend to multiple copying for promotional or commercial purposes. Code 0968-0446/2002 \$16.00. ISI Tear Sheet Service, 3501 Market Street, Philadelphia, PA 19104, USA, is authorised to supply single photocopies of separate articles for private use only. Organizations authorised by the UK Copyright Licensing Agency may also copy material subject to the usual conditions. For all other use, permission should be sought from Cambridge University Press.

No part of this publication may otherwise be reproduced, stored or distributed by any means without permission in writing from Cambridge University Press, acting for the copyright holder.

Copyright () The Natural History Museum, 2002

ELECTRONIC ACCESS This journal is included in the Cambridge Journals Online service which can be found at: http://journals.cambridge.org

For further information on other Press titles access http://uk.cambridge.org or http://us.cambridge.org

World list abbreviation: Bull. nat. Hist. Mus. Lond. (Bot.)

ISSN 0968-0446

The Natural History Museum

Cromwell Road

London SW7 5BD

Botany Series

Vol. 32, No. 1, pp. 1-59

Issued 27 June 2002

Typeset by Ann Buchan (Typesetters), Middlesex

Printed in Great Britain by Henry Ling Ltd, at the Dorset Press, Dorchester, Dorset 


\title{
Taxonomic notes on some African species in the family Calymperaceae (Musci)
}

\begin{abstract}
SyNOPSIS. Syrrhopodon usambaricus Broth. ex S. Orbán is placed in synonymy with Syrrhopodon asper Mitt., and Syrrhopodon lisowskii S. Orbán in synonymy with Syrrhopodon gardneri (Hook.) Schwägr. The distinctive features of Syrrhopodon stuhlmannii Broth. are discussed, and the only records of Calymperes moluccense Schwägr. from Africa are redetermined as Calymperes palisotii Schwägr.
\end{abstract}

The research for this paper was largely undertaken in response to difficulties encountered in identifying the specimens of Syrrhopodon collected during the British Bryological Society Expedition to Mulanje Mountain, Malawi, 1991. Determination of this material would have been considerably more difficult without the important primary accounts of the African species of Syrrhopodon by Orbán (1981) and Orbán \& Reese (1986).

Syrrhopodon asper Mitt. in J. Linn. Soc. Bot. 7: 151: 1863. Type: Tanzania, Kilimanjaro, Hannington s.n. (NY!-holotype, BM!isotype).

Fig. 1.

Syrrhopodon usambaricus Broth. ex S. Orbán in Acta Bot. Hung. 24: 113 (1978), syn. nov. Type: Tanzania, Usambara, Lutindi, 1902, Liebusch s.n. (H-BR!-holotype).

Discussion. The leaves in Syrrhopodon asper Mitt. consist of a linear-lanceolate chlorophyllose limb extending from a subelliptical hyaline base. They possess a prominent marginal rib and are spinulose to various degrees. As in many species of Calymperes and Syrrhopodon, the leaves in different specimens can vary widely in their relative dimensions (Fig. la-c), and range from a stubby $4 \mathrm{~mm}$ to a slender $7.5 \mathrm{~mm}$ long. The marginal ribs, viewed in cross-section, are well differentiated. Commonly, a superficial layer of chlorophyllose cells encloses small dorsal and ventral groups of stereids that are separated by a median row of guide cells (a costalike arrangement of tissues). This arrangement of cells in the marginal rib is plainly developed in the leaves of some specimens and less well developed in others (Fig. 1e-h). Towards the base in all leaves, the marginal rib becomes a flattened, undifferentiated, often unistratose band of linear cells. The region in the leaf base in which the margin transforms from a differentiated rib to an undifferentiated band also varies between specimens.

The type specimen of Syrrhopodon usambaricus Broth. ex S. Orbán (Liebusch s.n., H-BR) represents a form of $S$. asper Mitt. with tall, slender shoots. The leaves are relatively fine and narrow, and hardly curl when dry. The marginal ribs are well differentiated, and in the leaf base, the transition from polystratose rib to undifferentiated band occurs well below the apex of the hyaline lamina (Fig. 1i). In the isotype material of $S$. asper (Hannington s.n., BM) the shoots are small and have shorter, stubbier leaves. These curl when dry, are notably spinulose, and have prominent, well-differentiated marginal ribs. The region along the leaf at which the margin transforms from differentiated polystratose rib to unistratose/bistratose band tends to be adjacent to the apex of the hyaline lamina (Fig $1 \mathrm{k}$ ).
Intermediate expressions of these contrasting features of Hannington s.n. and Liebusch s.n. are apparent in the great range of material now available for examination, and indicate that these superficially distinct type specimens represent extreme forms of the same species. For example, Wood 1726 (Tanzania, BM, BM-K) has leaves proportioned like those of the type of Syrrhopodon asper that become curled when dry, but some cross-sections through the distal hyaline leaf base show a degree of differentiation closer to that in the type of S. usambaricus (Fig. 1j).

SPECIMENS EXAMINED. Malawi. Mulanje Mountain, June 1991: Hodgetts 2041a, 2047c, 2220b, 2532a, 2669a (RNG!); Kathumba M5915a (RNG!), M5916 (BM!); Kungu M3123a (RNG!); Longton M8054a, M8058a, 8425b (RNG!); Magombo M404lb, 4042b (RNG!); Porley 35a, 278a (RNG!); Russell M6055b, M6063a, M6068a (RNG!); Wigginton M1034a, M1682a (RNG!), M1201a (BM!). Uganda. Kadese, Ruwenzori Mts, above Miniba camp, 2700 m, 22 January 1962, Loveridge JPL397 (BM!). Kenya. Mutha Hill, August 1938, Boy Joana 7519 (BM!, BM-K!). Tanzania. Usambara, Lutindi, 1911, Liebusch s.n. (H-BR!); Usambara Ouset, crête Matundsi-Mashindei, SE of Ambangudu Tea Estate, 1300 m, 5 February 1985, Pócs 8533/R (BM!); Kilimanjaro: above Marangu, 2000 m, 13 July 1948, Hedberg 1144e (BM-K!); on path between Marangu and Bismark Hut, 2400 m, 24 February 1953, Wood 1726 (BM!, BM-K!). Morogoro District: Nguru Mts, ridge behind Dikurura Valley, 1700-1900 m, 602'S 37³2'E, Pócs 89119/W (BM!); Nguru ya Ndege Hill NNW of Morogoro town, summit, 1200-1350 m, 6 42’ S 37 36'E, Pócs \& Knox 88252/H (BM!). Mozambique. Namúli, Makua Country, 1887, Last s.n. (BM-K!).

Syrrhopodon stuhlmannii Broth. Bot. Jahrb. Syst. 24: 240 (1897).

Type. Tanzania, Uluguru, Bergwald, 1600 m, Stuhlmann 8809 (BM!-isotype).

Fig. $2 \mathrm{a}-\mathrm{d}$.

Discussion. In Orbán \& Reese (1986) Syrrhopodon usambaricus Broth. ex S. Orbán [=Syrrhopodon asper Mitt.] is keyed out beside S. stuhlmannii Broth. Large specimens of S. asper are superficially similar to those of $S$. stuhlmannii. Both species possess leaves with marginal ribs that have a costa-like structure (viewed in crosssection). However, the species are easily distinguished. In leaves of S. stuhlmannii the marginal ribs are mostly smooth; the cells of the chlorophyllose lamina are ventrally roundly protuberant, and dorsally flat to barely protuberant (Fig. $2 a-d$ ). The rib at the margin of the distal hyaline lamina is very well developed and strongly differentiated, with a median row of guide cells often more than nine cells wide (Fig. 2c, d). In contrast, most superficial cells of the marginal 


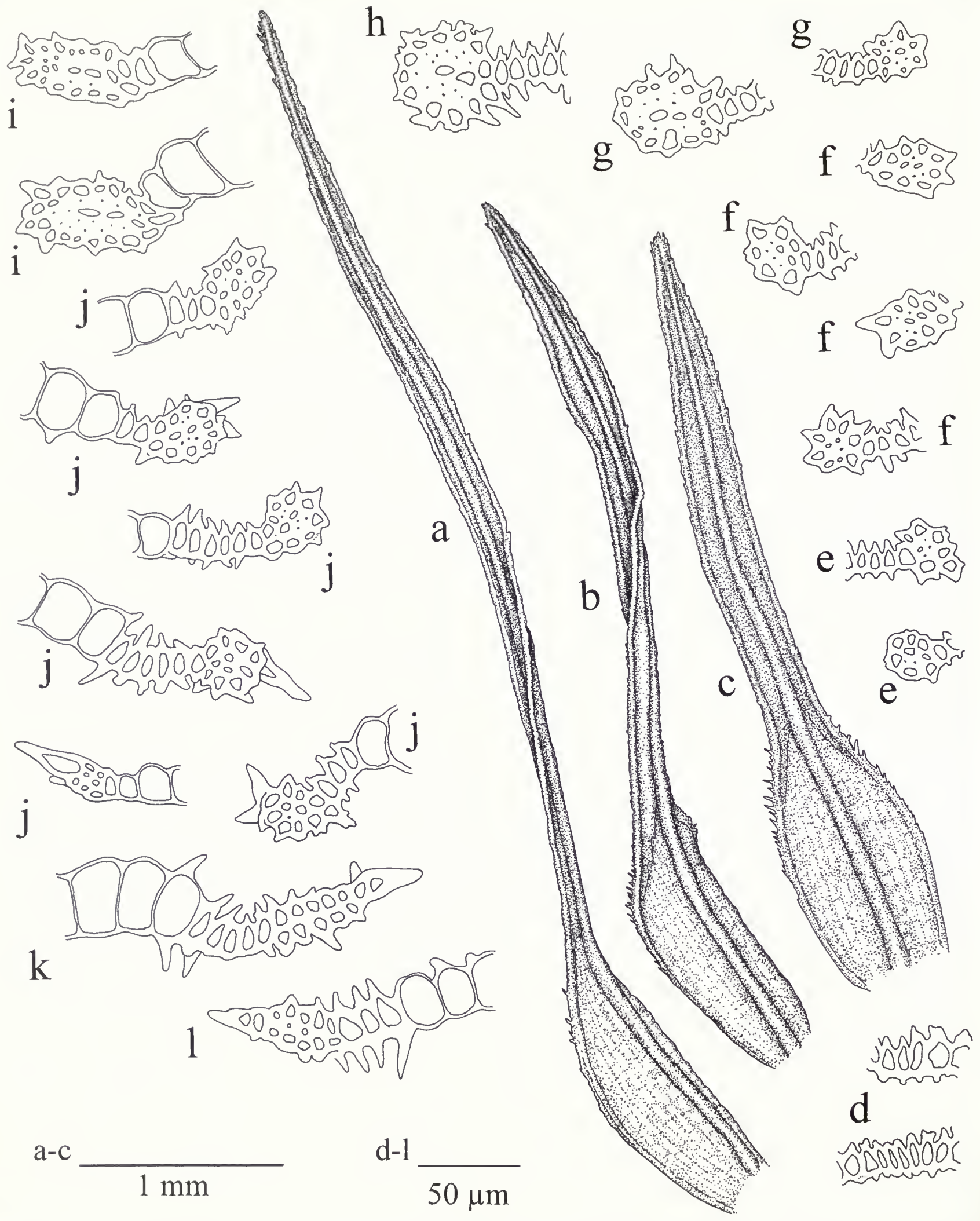

Fig. 1 a-l. Syrrhopodon asper Mitt. a-c: leaves; d-l: cross-sections of leaf through (d: chlorophyllose lamina, e-h: margin of chlorophyllose lamina, i-l: margin around distal hyaline lamina. a Drawn from Pócs 8533R (BM). b Drawn from Pócs \& Knox 88252/H (BM), c Drawn from Boy Joana 7519 (BM). d, g, i, Drawn from Liebusch s.n. (H-BR, holotype of Syrrhopodon usambaricus). e, j Drawn from Wood 1726 (BM). f, k Drawn from Hannington S.n. (BM, isotype of Syrrhopodon asper). h, I Drawn from Pócs 89119/W (BM). 

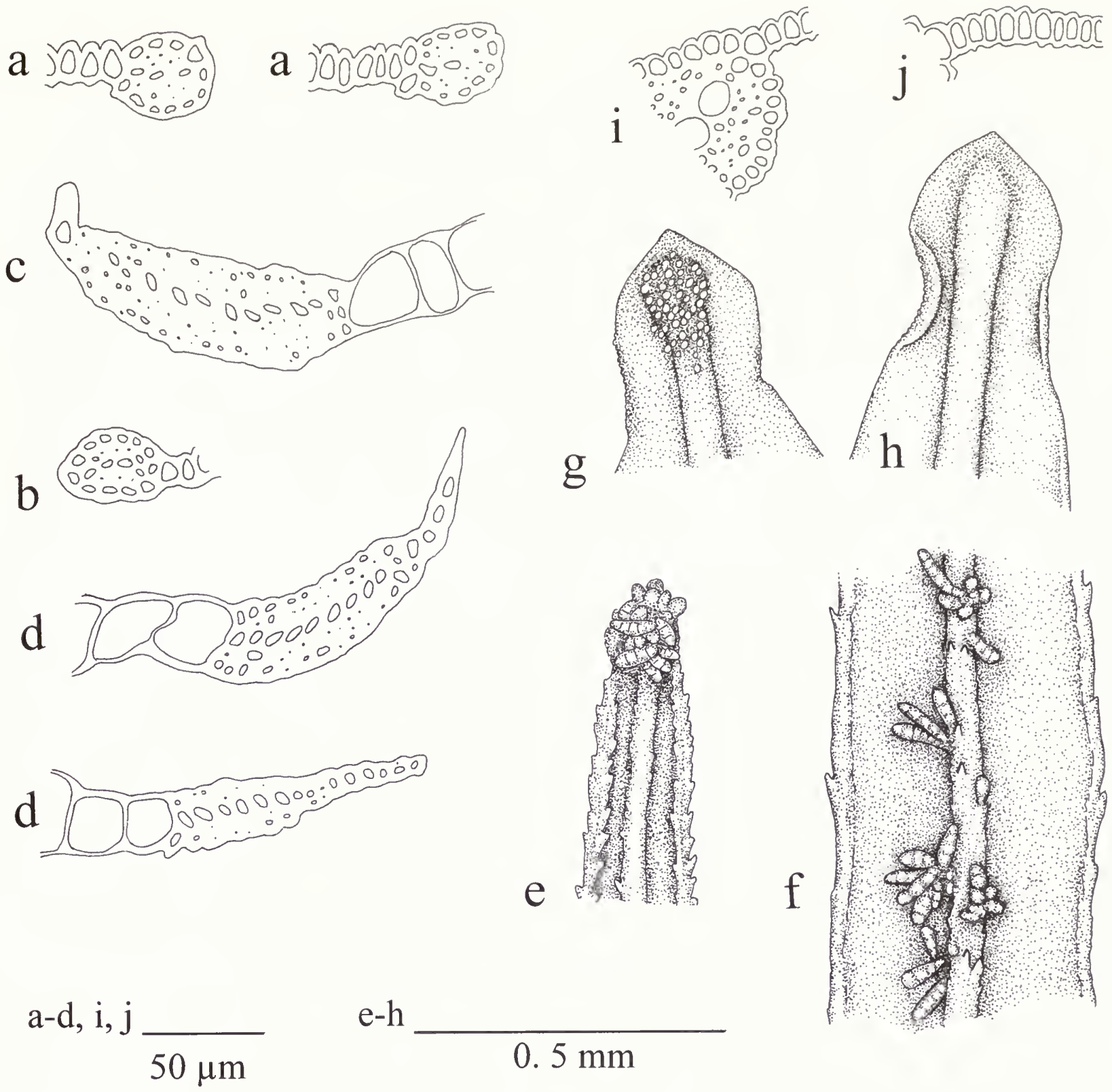

Fig. 2 a-d. Syrrhopodon stuhlmannii Broth. a-d: cross-sections through leaf margins (a, b: in chlorophyllose limb, c, d: in distal hyaline base). e, f: Syrrhopodon gardneri (Hook.) Schwägr. e, f: gemmae on leaves (e: in cluster at apex (ventral surface), f: in groups in mid-leaf). g-j. Calymperes palisotii Schwägr. $\mathrm{g}$, h: apices of gemmiferous leaves ( $\mathrm{g}$ : in ventral view showing gemmae-producing region (gemmae lost), h: in dorsal view); i, j: crosssections of chlorophyllose limb (i: showing half of costa, and j: lamina). a. c Drawn from Pócs \& Knox 89053/AK (BM). b, d Drawn from Stuhlmann 8809 (BM, isotype of Syrrhopodon stuhlmannii). e Drawn from Kathumba M5073b (RNG). f Drawn from Longtor M8375a (RNG). g, h, i, j Drawn from Lisowski 50255 (EGR).

ribs and the cells of the chlorophyllose lamina in $S$. asper are replete with acute projections (Fig ld-1). If apparent at all, the median row of guide cells in the rib at the margin of the distal hyaline lamina is usually less than six cells wide (Fig. 1i-1).

Syrrhopodon stuhlmanni Broth. remains a distinct species, apparently endemic to Tanzania.

SPECIMENS EXAMINED. Tanzania. Uluguru, Bergwald, $1600 \mathrm{~m}$, Stuhlmann 8809(BM!-isotype of S. stuhlmannii). Uluguru Mountains, 30 July 1941, Eccles AH8648 (BM!), AH8652 (BM!). Morogoro District. Nguru Mts, ridge above 'Spirit Lake' at the north source of
Chazi River above Chazi Falls, 2000-2100 m, $6^{\circ} 00^{\circ} \mathrm{S} 37^{\circ} 30^{\prime} \mathrm{E}, 4$ February 1989, Pócs \& Knox 89053/AK (BM!); Lushoto District, West Usambara Mts, $5 \mathrm{~km}$ east of Mgwashi village on west slope of Gonja Hill, 1600-1700 m, 4 47'S 38³3'E, Pócs \& Krog 88205/R (BM!).

Syrrhopodon gardneri (Hook.) Schwägr., Sp. musc. frond. suppl. 2 (1): 110 (1824).

Fig. 2e, f, 3 ,

Calymperes gardneri Hook. Musci Exot. 2: 146 (1819). Type: Nepal, Gardner [1205] (BM!-holotype, BM!-isotype). 

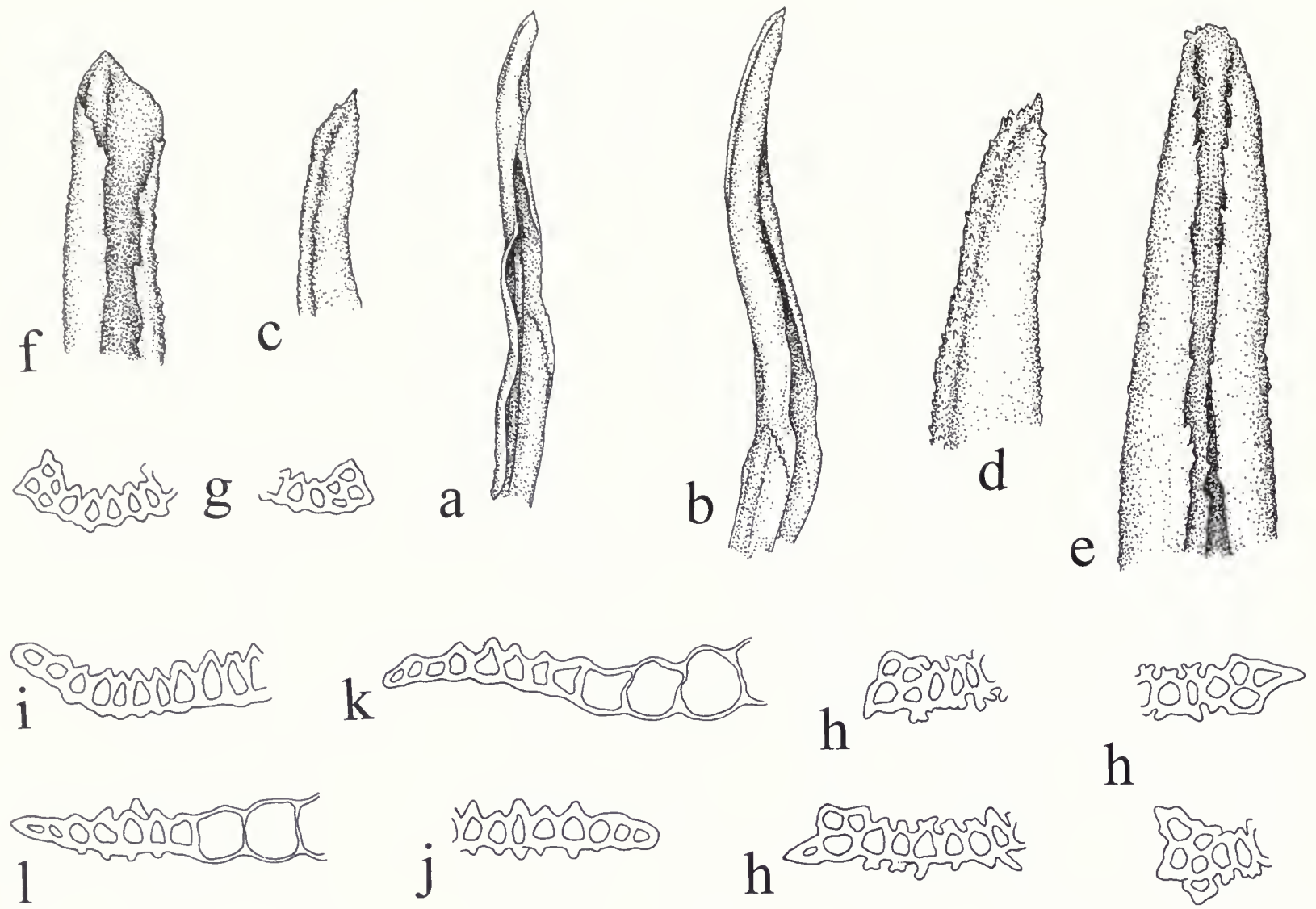

h
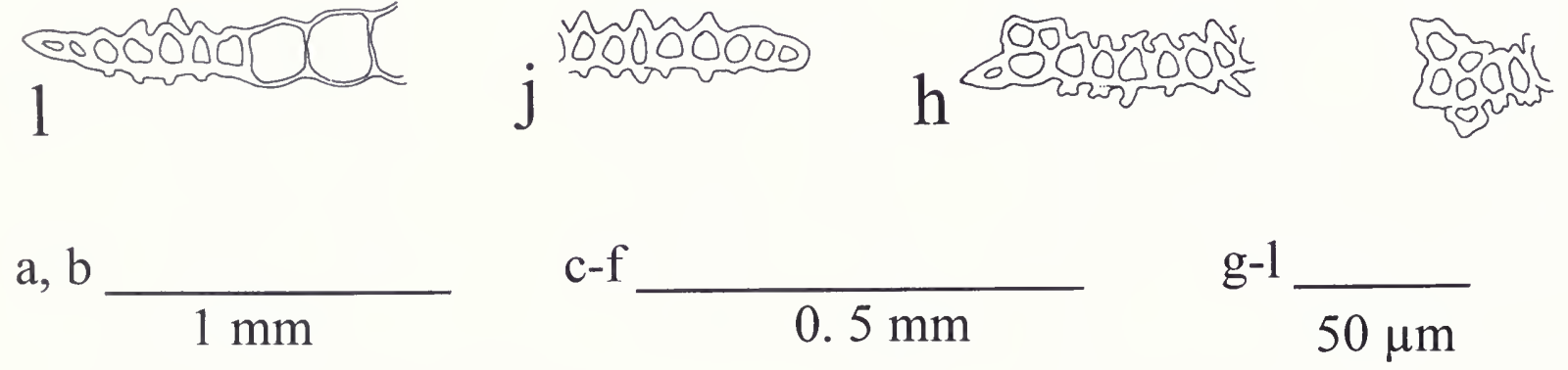

Fig. 3 a-l. Syrrhopodon gardneri (Hook.) Schwägr. a-b: leaves; c-f: details of leaf apex, (c, d: in lateral view, e, f: in ventral view); g-l: cross-sections through leaf margin (g, h: in chlorophyllose limb, i, j: around apex of hyaline base, k, l: in distal hyaline base). a, c, f, g, i, k Drawn from Gardner HI205 (BM, isotype of Syrrhopodon gardneri). b, d, e, h, j, l Drawn from Lisowski s.n.(EGR, holotype of Syrrhopodon lisowskii).

Syrrhopodon lisowskii S. Orbán in Egri Ho Si Minh Tanárképzö Föis. Füzetei 18: 81 (1987), syn. nov. Type: Zaire, Haut Shaba, Env. de Kasumbalesa, Colline Kibwe I., 1400 m, 20 March 1971, Lisowski s.n. (EGR!-holotype).

Discussion. The holotype of Syrrhopodon lisowskii S. Orbán possesses narrow, gemma-bearing leaves that have strongly incurved margins. Orbán \& Reese (1986) use these features to distinguish $S$. lisowskii from $S$. gardneri (Hook.) Schwägr. However, cross-sections of the leaves from the types of $S$. gardneri and S. lisowskii are indistinguishable (Fig. 3-1), and an examination of a large number of specimens has shown that leaves with incurved margins and/or bearing gemmae fit comfortably within the range of features characteristic of $S$. gardneri.

In the latter species, young leaves often tend to possess strongly incurved margins and can have blunt, almost cucullate apices. The isotype material of Syrrhopodon gardneri (BM) has many leaves with a lamina approaching the degree of incurvature apparent in $S$. lisowskii (Fig. 3e, f). Gemmae occur in many specimens of $S$. gardneri and their presence is not correlated with the relative breadth of the leaf or incurvature of the leaf margins. They may be produced on the ventral and sometimes dorsal surfaces of the costa, often in a bunch at the leaf apex (Fig. 2e). In a collection from Malawi (Longton M8375a) gemmae occur in small groups at intervals along the costa, associated with the loosely transverse rows of prominent costal teeth. The leaves of this specimen are not especially narrow nor are the margins of the chlorophyllose lamina particularly incurved (Fig. 2f). In all respects it is identifiable as $S$. gardneri.

SPECIMENS EXAMined. Malawi. Mulanje Mountain, June 1991: $N$. Hodgetts M2008e, M2026d, M2048b, M2066a, M2067b, 2383a, 2555a, M2638a, 266la, 2662c (RNG!); Kathumba M5058(BM!), M5073b(RNG!); Kungu M3241 (BM!), M3267c, M3268a, M3033 (RNG!); Longton M8231a, M8232a, M8375a, M8648a (RNG!); Magombo M4238a (RNG!); O'Shea 7107b, 7108d, 7110a, 7111b, 7138a, 240a, 7240b pro parte, 724lb, M7404c, M7406b, M7508a, M7509b (RNG!), 7318a, 7389a (BM!); Porley 98a, 232a, 317a, 329a (RNG!); Wigginton M1009b pro parte, M1024b pro parte, M1217a, M1219a, M1225a, M1243a, M1247a, M1417a, M1576a (RNG!). Zomba Plateau, forestry campsite opposite Chawe School, 1470 m, 8 August 1993, Stevenson s.n. (BM!). Uganda. Ishasha 
Gorge, Kanunga, 7500 ft, 6 June 1952, Lind 14 (BM!); Bwindi National Park, forest $1 \mathrm{~km}$ east of the Zaire border near Rukubira, 1680 m, 059’53"S 29³5’ 50"E, 3 February 1996, Matchain UI 118 a (BM!). Democratic Republic of Congo. Lushiji, October 1923, Overlaet 347 (BM!). Angola. Huilla District, near Humpata, 3800$5500 \mathrm{ft}$, May 1860, Welwitsch 6 (BM!- isotype of Calymperes welwitschii Dub.). Madagascar. Ankafana, 1880, Deans Cowan s.n. (BM!).

Calymperes palisotii Schwägr. Sp. musc, frond. suppl. 1 (2): 334 (1816). Type: Nigeria, 'Oware' [Warri], Palisot de Beauvois s.n. (BM!- lectotype).

Fig. $2 \mathrm{~g}-\mathrm{j}$.

Discussion. Orbán (1995) identified three specimens from continental Africa as Calymperes palisotii ssp. moluccense (Schwägr.) M. Menzel in M. Menzel \& Schultze-Motel [=Calymperes moluccense Schwägr.]. These specimens, collected by Lisowski from Zaire and Guinea, would have been the first African records for C. moluccense. Unfortunately, all three collections are Calymperes palisotii Schwägr. (non $C$. moluccense). The cells of the chlorophyllose lamina are ventrally roundly protuberant (Fig. 2i, j) and the lamina at the apex of gemmiferous leaves forms a broad point (Fig. 2g, h). These features are typical of $C$. palisotii, and are especially well represented in collections from Africa. As explained by Ellis (1987) and Ellis \& Tan (1999), C. moluccense (syn. C. palisotii ssp. moluccense) is distinguishable from $C$. palisotii by its possession of laminal chlorocysts that are ventrally drawn out as acute, often coronate-papillose projections, and gemmiferous leaves with a rounded rather than broadly pointed apex.

Calymperes moluccense Schwägr. has yet to be found in continental Africa or the adjacent islands of the Indian Ocean, and must still be regarded as an Indo-Pacific species.

SPecimens eXAmined. Guinea. Conakry, Campagnie Minière, 23 December 1961, Lisowski 918 (EGR!). Democratic Republic of Congo. Haut-Zaire, Kisangani: près de la Porte, 11 December 1977. Lisowski 50255 (EGR!); centre de la ville, 22 December 1977, Lisowski 50379 (EGR!).

ACKNOWLEDGEMENTS. I am grateful to the directors and staff of EGR (especially Dr S. Orbán), NY and RNG for the loan of specimens.

\section{REFERENCES}

Ellis, L.T. 1987. Taxonomic notes on Calymperes. Journal of Bryology 14: 68 I-690. _ \& Tan, B.C. 1999. The moss family Calymperaceae (Musci) in the Philippines. Bulletin of The Natural History Museum, London (Botany Series) 29 (I): 1-46.

Orbán, S. 1981. Studies on African Calymperaceae, III. Conspectus of the African species of Syrrhopodon Schwaegr. Acta Botanica Hungarica 27 (I-2): I69-I77.

1995. Studies on African Calymperaceae, VI. New data to continentaI Africa and Madagascar. Acta Botanica Hungarica 39 (3-4): 227-234.

_ \& Reese W.D. 1986. Notes on the taxonomy of African Syrrhopodon. Abstracta Botanica 10: 349-255. 



\title{
Typification of Ranunculus bullatus L. (Ranunculaceae)
}

\author{
RICCARDO M. BALDINI \\ Museo Botanico, Università degli Studi di Firenze,via G. La Pira 4, I-50I21 Firenze, Italy; rbaldo@unifi.it \\ CHARLES E. JARVIS \\ Department of Botany, The Natural History Museum, Cromwell Road, London SW7 5BD; c.jarvis@nhm.ac.uk
}

\begin{abstract}
SyNOPSIS. The typification of Ranunculus bullatus L. is discussed and a lectotype and epitype chosen which maintain usage of the name in the sense of subsp. bullatus. Ranunculus bullatus subsp. cytheraeus (Halácsy) Vierh. is recognized at species rank, and the combination $R$. cytheraeus (Halácsy) Baldini is made to accommodate it. Both taxa are described and illustrated. The names $R$. bullatus L. var. cuneifolius Coustur. \& Gand., R. bullatus L. var. cyrenaicus Pamp., $R$. bullatus L. var. ovatus Freyn, $R$. bullatus L. var. prolifer Gennari, R. semicalvus Jord. \& Fourr. and Ionosmanthus plantagineus Jord. \& Fourr. are also typified.
\end{abstract}

\section{INTRODUCTION}

Ranunculus bullatus L., s. l., is a polymorphic taxon distributed in the Mediterranean area (Greuter et al., 1989; Jalas \& Suominen, 1989) by scattered populations from Portugal to Greece and Northern Africa (Maire, 1964). It is well-known that there are many difficulties in distinguishing the several infraspecific taxa described within this species (Gandoger, 1889; Vierhapper \& Reichinger fil., 1935; Davis, 1965) because many diagnostic characters overlap. As a part of a wider study of this group of taxa in Europe (Baldini, in prep.), it is necessary to establish the correct application of the Linnaean name.

\section{LECTOTYPIFICATION OF RANUNCULUS BULLATUS L.}

Linnaeus (1753: 550) used a diagnosis 'Ranunculus foliis ovatis serratis, scapo nudo unifloro' cited from his earlier Hortus Cliffortianus (Linnaeus, 1738) along with three synonyms from, respectively, Bauhin (1623), Dodoens (1583) and Clusius (1601). He also recognized one unnamed variety (var. $\beta$ ) based on a series of polynomials of Morison (1680) ' $\beta$. Ranunculus latifolius bullatus autumnalis, flore pleno \& prolifero. Moris. hist. 2. p. 447. s. 4. t. 31. f. 49. 50. 51.'. Finally, Linnaeus added 'Habitat in Lusitania, Creta', and a symbol to indicate a perennial life form (Fig. 1).

Although there are two sheets referred to this taxon in Linnaeus' Herbarium at LINN (numbering follows Savage, 1945), 715.10 lacks the relevant Species Plantarum number (i.e. ' 8 ') and is therefore a post-1753 addition to the herbarium (see Turland \& Jarvis, 1997: 458-459), and 715.11 is entirely unannotated. We therefore do not accept either sheet as original material for this name, although both are identified (e.g. by Davis, 1965: 179) as belonging to the typical subspecies. There is no relevant material in any of the other 'general' Linnaean collections (e.g. H, MW, S, SBT, UPS), nor is there any in the Burser Herbarium (UPS) which can be associated with the Bauhin polynomial cited by Linnaeus. However, there is a specimen in the Clifford Herbarium (Herb. Clifford: 229, Ranunculus 7, BM! (viewable at http://www.nhm.ac.uk/botany/databases/ clifford)) which can be associated with both var. $\beta$ of Hortus Cliffortianus (Linnaeus, 1738) and var. $\beta$ of Species Plantarum
(Linnaeus, 1753) and is a double-flowered form ('flore pleno \& prolifero') of $R$.. bullatus. It is similar to the plant portrayed by Cornut (1635: t. 95; cited by Linnaeus as a synonym in 1738 but omitted in 1753) and Morison (1680: s. 4, t. 31, f. 50). The Clifford specimen is original material for the name.

The other original elements for the name are drawn from the cited and uncited illustrations. The woodcut of Dodoens' (1583: 426) 'Ranunculus Lusitanicus' was copied by Morison (1680: s. 4, t. 31, f. 51 , as 'Ranunculus latifolius autumnalis caule hirsuto flore minimo, nobis') via the uncited Clusius (1601: 238, as 'Ranuncul. grumosa rad. I. species $I I^{\prime}$ ) and represents a plant from Crete (notwithstanding Dodoens' belief that the plant came from Portugal) identifiable as $R$. bullatus subsp. cytheraeus (Hal.) Vierh. (see Greuter et al., 1989: 428).

Clusius' (1601: 238) 'Ranunculus grumosa radice I.', also copied by Morison (1680: s. 4, t. 31, f. 49, as 'Ranunculus latifolius autumnalis fl. simplici, nobis'), represents a plant from the Iberian Peninsula, identifiable as $R$. bullatus $\mathrm{L}$. subsp. bullatus.

Lastly, for his unnamed variety $\beta$, Linnaeus cited Morison's (1680: s. 4, t. 31, f. 49, 50 and 51) along with a single, composite polynomial that Linnaeus derived from those used by Morison for his three illustrations (for the identities of these, see above).

There are therefore six possible elements from which a lectotype could be chosen: the Clifford material, the element represented by Dodoens' illustration, that represented by Clusius' figure, and the three Morison figures associated with variety $\beta$. Clifford's material and Morison's f. 50 have petaloid staminodia ( $(.$. flore pleno \& prolifero'), are almost certainly of cultivated origin and would not effectively restrict the application of subsp. bullatus. Dodoens' illustration and Morison's f. 51 belong to subsp. cytheraeus so either would make an unfortunate and disruptive type choice. However, Clusius' illustration (copied by Morison as his f. 49) belongs to subsp. bullatus and 'Ranınculus grumosa radice I.' in Clusius, Rariorum plantarum historia: 238 (1601) is therefore designated here as the lectotype of Ranunculus bullatus L. (Fig. 2). Given its somewhat schematic nature, it seems wise to designate an epitype to establish its application beyond any possible doubt, which we do here (see Taxonomic Summary).

Study of the variation in Ranunculus bullatus L. s.l. by the senior author (Baldini, in prep.), leads to the conclusion that $R$. bullatus $\mathrm{L}$. subsp. cytheraeus (Halácsy) Vierh. is best recognized at species rank. This is based on several distinct features, including flower size 
bullatws. 8. RANUNCULUS foliis ovatis ferratis, fapo nudo unifioro. Hort. chff. 229 .

Ranunculus latifolius bullatus, afphodeli radice. Basb. pin. 18r.

$\mathrm{R}$ aнunculus lufitanicus. Dod. pempit. 429.

Rarunculus grumofa radice I. Clkf. bift. I. p. 238.

B. Rannnculus latifolius bullatus autumnalis, fore plenn \& proliticro. Morif. bijt. 2. p.447. S.4.t. 31.f.49.50. $5 \mathrm{t}$.

Habitiat in Lufitania, Crçta, 2

Fig. 1 The protologue of Ranunculus bullatus Linnaeus, Species Plantarum: 550 (1753).

\section{Ranuncul. grumofaradice 1.}

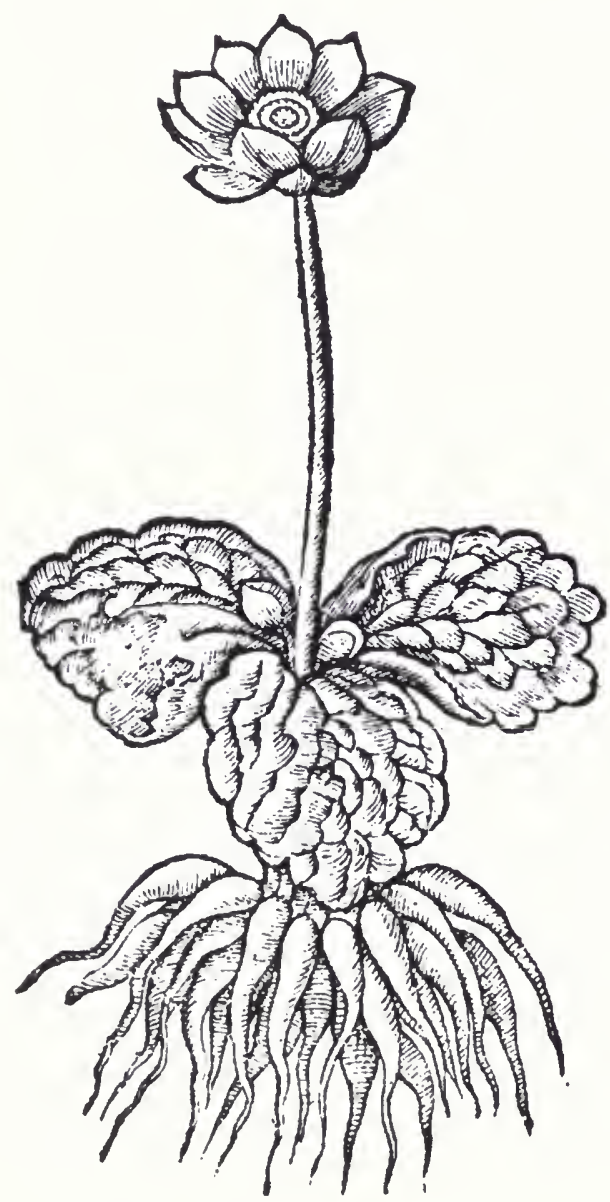

Fig. 2 'Ranunculus grumosa radice I.' in Clusius, Rariorum plantarum historia: 238 (1601), the lectotype of Ranunculus bullatus L.

and arrangement, leaf morphology and habit, and their correlation with geographical distribution. A new combination, relevant synonymy, key and species descriptions and illustrations are given below.

\section{TAXONOMIC SUMMARY}

\section{Key to Ranunculus bullatus and $R$. cytheraeus}

1 Leaf lamina ovate, crenate, never mucronate, more or less bullate and pubescent, sometimes glabrous or hispid above or beneath; scape to 20 $25 \mathrm{~cm}$ long, never branched, more or less pubescent, l-flowered; achenes with a short, curved beak 1. R. bullatus

Leaf lamina obovate to elliptical, crenate-denticulate, mucronate, upper surface villose especially at the margin; scape to $8 \mathrm{~cm}$ long, branched with small bracteole at the base of each branch, with spreading hairs, $1-$ 2-flowered; achenes with a short, uncinate beak ...... 2. R. cytheraeus

1. Ranunculus bullatus L., Sp. pl. 1: 550 (1753). Type: 'Ranunculus grumosa radice I.' in Clusius, Rar. pl. hist.: 238 (1601), lectotype, designated here. Epitype: 'Flora Lusitanica. Prov. Estremadura, ad pagum, 1848, leg. Dr. Welwitsch 198' (BM!), designated here.

Fig. 3.

Ranunculus supranudus Jord. \& Fourr., Brev. Pl. Nov. 1: 1 (1866). Type: 'Algeria: Daroussa prope Bône, ex dom. A. Joannon'. Not located.

R. semicalvus Jord. \& Fourr., Brev. Pl. Nov. 1: 2 (1866). Type: 'Ajaccio, 15 octobre 1866, col. De Marsilly 101 bis' (P!, lectotype, designated here; BM!, K!-isotypes).

R. bullatus L. var. prolifer Gennari, Spec. e var. Fl. Sard.: 29 (1866), non Cavara \& Grande in Boll. Soc. Bot. Ital.: 101 (1925). Type: 'Ranunculus bullatus scapo diviso seu prolifero, Cagliari ai Cappuccini, 9bre. 1863, Gennari s. n.' (TO!- lectotype, designated here).

Ionosmanthus semicalvus (Jord. \& Fourr.) Jord. \& Fourr., Icon. Fl. Eur. 2: 14 (1869).

I. plantagineus Jord. \& Fourr., Icon. Fl. Eur. 2: 14 (1869). Type: 'Algeria, Costantine, tab. CCXLIV. - 326' in Jordan \& Fourreau, Icon. Fl. Eur. 2. (1869), lectotype, designated here.

R. bullatus L. var. $\alpha$ ovatus Freyn in Willk. \& Lange, Fl. Hisp. Prodr. 3: 919 (1880). Type: 'Malaga, Willkomm exsicc. 1844, n. 418', (BM!-lectotype, designated here; G!-isotype).

R. bullatus L. var. $\beta$ bellidifolius Freyn in Willk. \& Lange, Fl. Hisp. Prodr. 3: 919 (1880). Type: 'Malaga, Kalisch'. Not located.

R. bullatus L. subsp. supranudus (Jord. \& Fourr.) Vierh. in Österr. Bot. Z. 84: 129 (1935).

$R$. bullatus L. subsp. bullatus var. plantagineus (Jord. \& Fourr.) Maire in Jahand. \& Maire, Cat. Pl. Maroc: 994 (1940).

R. bullatus L. subsp. bullatus var. supranudus (Jord. \& Fourr.) Maire in Jahand. \& Maire, Cat. Pl. Maroc: 994 (1941).

R. bullatus L. subsp. eu-bullatus Maire, Fl. Afrique N. 11: 139 (1964).

Perennial herb with fibrous roots and fusiform tubers; leaves (2-)3$5(-7)$ in basal rosette; petiole $2-5(-7) \mathrm{cm}$ long, with silky hairs; lamina ovate, crenate, sometimes crenate-denticulate, never mucronate, more or less bullate and pubescent, sometimes glabrous or hispid above or beneath, (1-)2-8 $\times 1-5(-6) \mathrm{cm}$; scapes erect, never branched, up to $20-25 \mathrm{~cm}$ long, more or less pubescent, sometimes glabrous, 1-flowered; flowers $1-2(-3) \mathrm{cm}$ in diameter, with 5 green sepals $0.3-0.6 \mathrm{~cm}$ long and 5-10(-12) yellow and obovate petals 0.5-1.5 cm long; receptacle glabrous, not elongated; achenes glabrous, ovate-inflated with a short and curved beak.

\section{DISTRIBUTION}

EUROPE. Tutin (1964: 234); Greuter et al. (1989: 428); Jalas \& Suominen (1989: map no. 1839); Tutin \& Akeroyd (1993: 281). Portugal: Pereira 


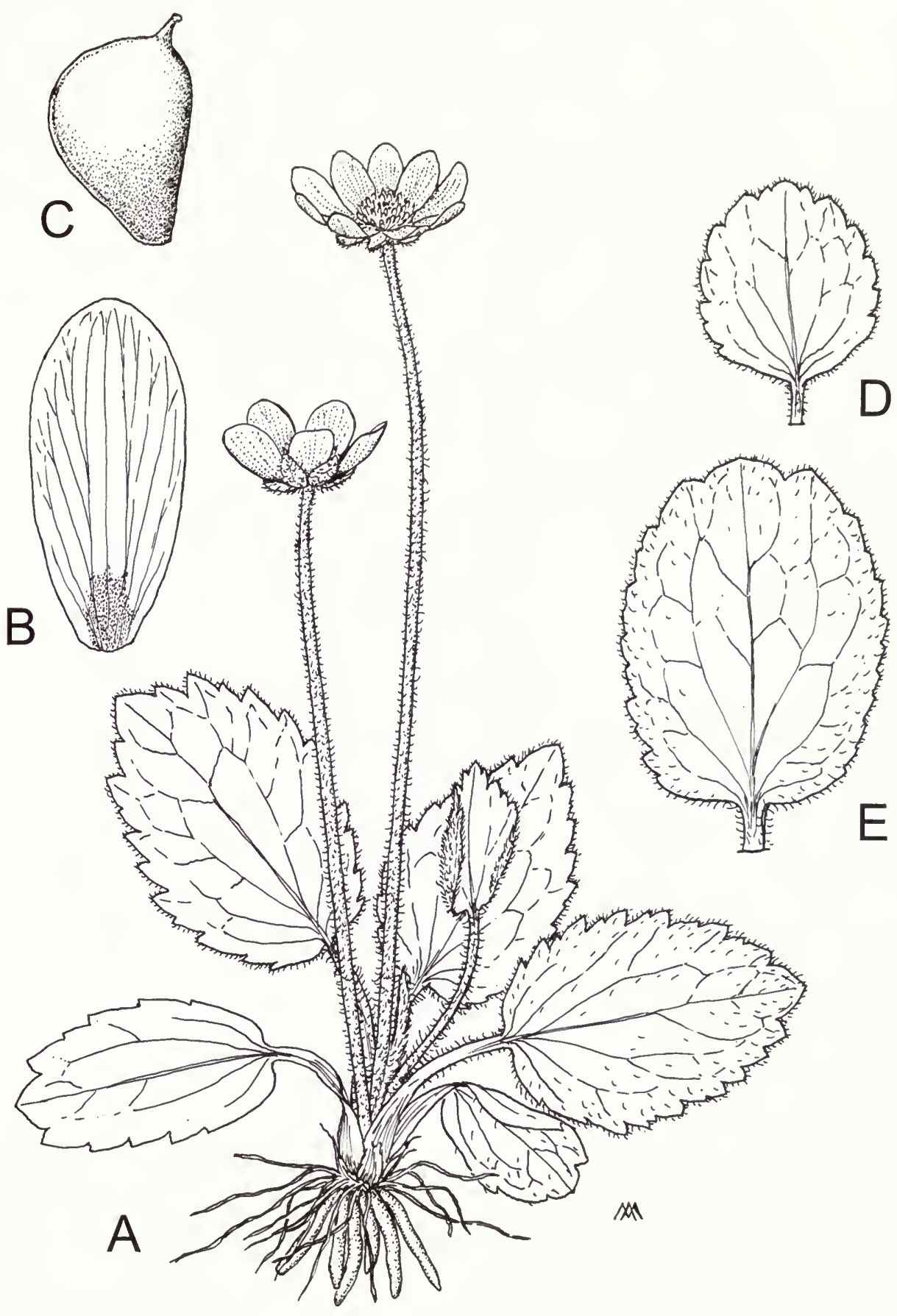

Fig. 3 Ranunculus bullatus L. From the epitype specimen, Welwitsch $198(\mathrm{BM})$, A. Maury delineavit. A. whole plant $\times 1$; B. petal $\times 4$; C. achene $\times 20$; the additional leaves $(x$ 1) show two of the more frequent foliar morphotypes found in this variable species. The upper leaf (D) is from a collection from the Balearic Islands (Bianor 2518, BM!); the lower leaf (E) is from a collection from Sicily (Ross 103, G!). 

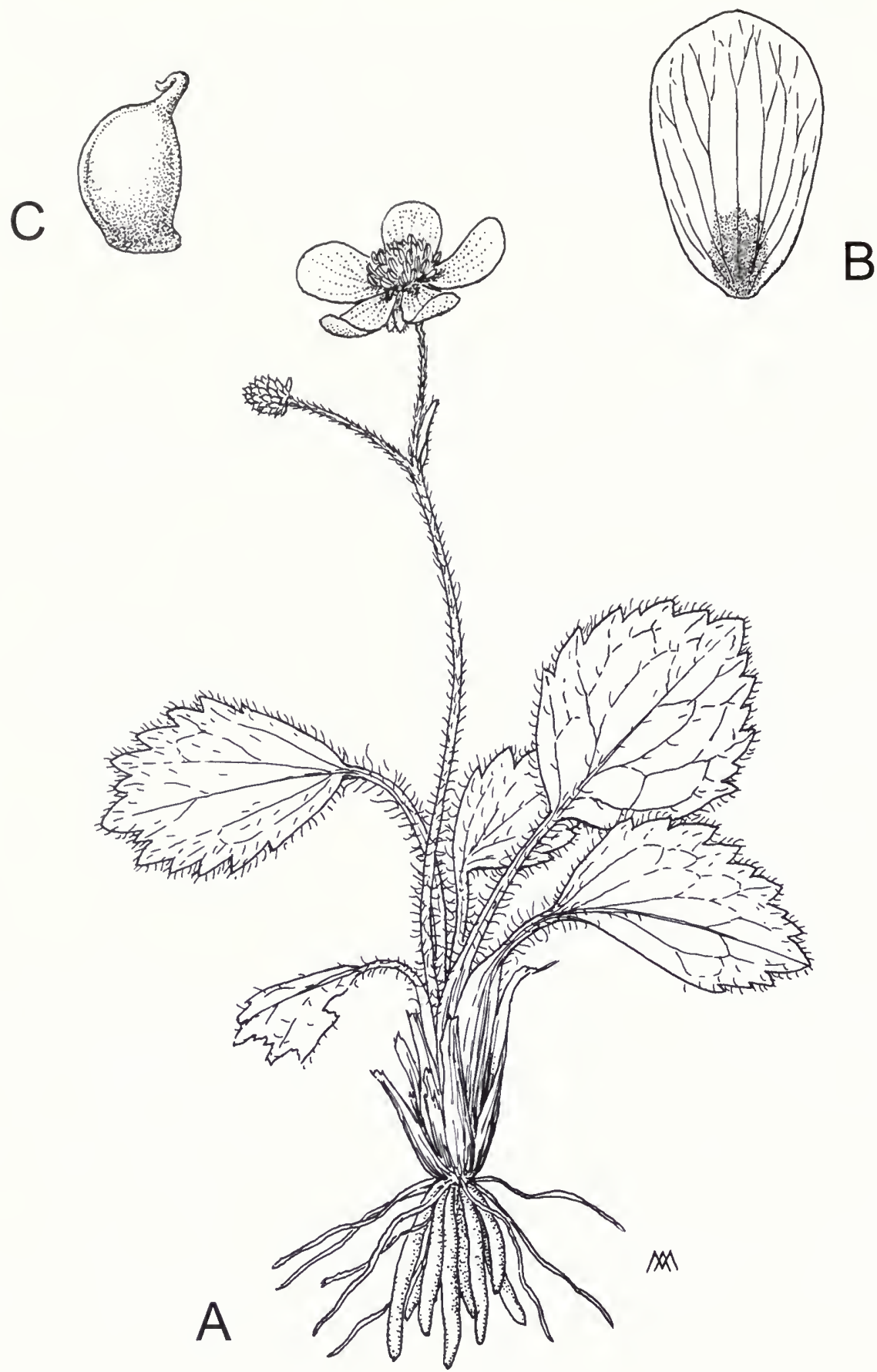

Fig. 4 Ranunculus cytheraeus (Halácsy) Baldini. From material from Cyprus (Agia Napa distr., Famagusta, 15 Dec. 1940), Davis 2052 (BM), A. Maury delineavit. A. whole plant $\times 2$; B. petal $\times 5$; C. achene $\times 20$.

Coutinho (1974: 279). Spain: López González (1986). Balearic Islands: Smythies (1984: 448). Corsica: Paradis et al. (2000). Italy (incl. Sardinia and Sicily): Fiori (1898: 506; 1924: 666); Zangheri (1976: 161); Pignatti (1982: 318). Crete: Turland et al. (1993: 132 sub $R$. bullatus L. p.p.). Turkey: Davis (1965: 179). Cyprus: Meikle (1977: 48).

NORTH AFRICA. Maire (1964: 137 excl. Libya where only R. cytheraeus (Halácsy) Baldini occurs). Morocco: Battandier (1888: 10); Jahandiez \& Maire (1932: 250); Nègre (1961: 241). Algeria: Battandier \& Trabut (1904: 12); Quézel \& Santa (1962: 370). Tunisia: Battandier \& Trabut (1904: 12); Pottier-Alapetite (1979: 156).
2. Ranunculus cytheraeus (Halácsy) Baldini, stat. nov.

Ranunculus bullatus L. var. cytheraeus Halácsy in Vierh., K. K. Zool.-Bot. Ges. Wien 54: 483 (1904). Type: 'Ranunculus bullatus L. v. cytheraeus. Foliis grosse inciso-dentatis, sparse pilosis subglabrisve, petalis 5, obovatis vel obovato-cuneatis. Ins. Cythera: in dumosis siccis reg. inf., 25.XI.1903, Chr. Leonis 5' (LY!lectotype, designated here; LY!-isotypes).

Fig. 4.

R. bullatus L. var. cuneifolius Coustur. \& Gand. in Bull. Soc. Duffour: 
36 (1914). Type: 'Ranunculus bullatus L., Spec., 774. V. cuneifolius Cousturier et Gandoger. Var. nov.! Cat. Cam. 37 - var. Crète: Candie. Dans les collines au nord d'Hagios-Déka. Décembre 1913, P. Cousturier 1479' (LY!-lectotype, designated here; MPU!isotype).

R. bullatus L. forma cuneifolius (Coustur. \& Gand.) Coustur. \& Gand. in Bull. Soc. bot. France 63: 13 (1916).

R. bullatus L. var. cyrenaicus Pamp. in Nuovo Giorn. Bot. Ital., n. s., 26: 212 (1919). Type: 'Ranunculus bullatus L. var. cyrenaicus Pamp., 5 Gennaio 1917: in fioritura, depressione del terreno fra le due Palme e la linea ferroviaria di Benina in direzione del Giok $($ Kebir $)$. Arabo $=$ Ain el noogia $=$ occhio di pecora. Gli arabi ne raccolgono i fiori e li uniscono alla mastice ricavata dalla pianta Hellek (Periploca laevigata) detta Elek, per darle il colorito giallo. Zanon 24' (FI!-lectotype, designated here).

R. bullatus L. var. prolifer sensu Cavara \& Grande in Bull. Soc. Bot. Ital.: 101 (1925), non Gennari, Spec. e var. Fl. Sard.: 29 (1866).

R. bullatus L. subsp. cytheraeus (Halácsy) Vierh. in Österr. Bot. Z. 84: 129 (1935).

R. bullatus L. subsp. cyrenaicus (Pamp.) Maire, Fl. Afrique N. 11: 139 (1964).

Perennial herb with fibrous roots and fusiform tubers; leaves 3-5(9) in basal rosette, with silky hairs; pedicels $0.5-1.5(-2.5) \mathrm{cm}$ long; lamina from obovate to elliptical, crenate-denticulate, mucronate, upper surface villose especially at the margin and somewhat pubescent beneath; scapes erect, $3-7(-8) \mathrm{cm}$ long, with spreading hairs, branched with small bracteole at the base of branch; flowers 1-2, $(0.5-) 1-1.5(-2) \mathrm{cm}$ in diameter, with 5 green sepals $0.3-0.5(-0.7)$ $\mathrm{cm}$ long and 5(-6) yellow and obovate petals, (0.5-)0.8-1.2(-1.5) $\mathrm{cm}$ long; receptacle glabrous and elongated; achenes glabrous, $0.1-$ $0.3 \mathrm{~cm}$ long, inflated with a short and uncinate beak.

\section{DISTRIBUTION}

GREECE. Cythera: Halácsy (1904 sub $R$. bullatus L. var. cytheraeus Halácsy); Davis (1965: 179 sub R. bullatus subsp. cytheraeus (Halácsy) Vierh.). Cyprus: Davis (1965: 179 sub R. bullatus subsp. cytheraeus (Halácsy) Vierh.); Meikle (1977: 48 sub R. bullatus subsp. cytheraeus (Halácsy) Vierh.). Crete: Rechinger (1943: 188 sub R. bullatus L. var. cuneifolius Coustur. \& Gand. p.p. \& var. cytheraeus Halácsy in Vierh. p.p.); Barclay (1986: 82 sub $R$. bullatus L. p.p.); Turland et al. (1993: 132 sub R. bullatus L. p.p.).

NORTH AFRICA. Libya: Béguinot \& Vaccari (1912a, 1912b sub $R$. bullatus sensu Bég. \& Vaccari, non L. (1753); Pampanini \& Zanon (1919: 212 sub $R$. bullatus L. var. cyrenaicus Pamp.); Pampanini (1931: 203 sub $R$. bullatus L. var. cyrenaicus Pamp.); Cavara \& Grande (1925 sub $R$. bullatus var. prolifer sensu Cavara \& Grande, non Gennari (1866); Maire (1964: 139 sub R. bullatus L. subsp. cyrenaicus (Pamp.) Maire; Davis (1965: 179 sub $R$. bullatus L. subsp. cytheraeus (Halácsy) Vierh.); Qaiser (1984: 35 sub $R$. bullatus L. subsp. cytheraeus (Halácsy) Vierh.).

ACKNowledgements. We are grateful to the curators of the herbaria cited above for permission to study the collections in their care, and in particular to Prof. P. Schafer (MPU). We are also grateful to Giorgio Padovani (Museo Botanico dell'Università degli Studi di Firenze) and Laura Settesoldi (Erbario Tropicale di Firenze) for their kind assistance in transferring images to $\mathrm{CD}$. CEJ acknowledges generous grant support to the Linnaean Plant Name Typification Project by the Linnean Society of London.

\section{REFERENCES}

Barclay, C. 1986. Crete. Checklist of the vascular plants. Englera 6: I-138.

Battandier, J.-A. 1888. Dicotylédones 1. Thalamiflores. In J.-A. Battandier \& L. Trabut (Eds), Flore de l'Algérie. Alger.

— \& Trabut, L. 1904. Flore analytique et synoptique de l'Algérie et de la Tunisie. Alger.

Bauhin, C. 1623. Prodromus theatri botanici. Basel.

Béguinot, A. \& Vaccari, A. 1912a. Contributo alla flora della Libia. Monografie e Rapporti Coloniali 16: 1-70. 10 plates. Roma.

— \& 1912b. Specie nuove o rare per la Flora della Libia. Padova.

Cavara, F. \& Grande, L. 1925. Altre piante rare o nuove della Cirenaica. Bollettino della Società Botanica Italiana: 100-105.

Clusius, C. 1601. Rariorum plantarum historia. Antverpiae.

Cornut, J.-P. 1635. Canadensium plantarum. Paris.

Davis, P.H. 1965. Ranunculus. In P.H. Davis (Ed.), Flora of Turkey and the East Aegean Islands 1: 146-195. Edinburgh.

Dodoens, R. 1583. Stirpium historiae pemptades sex, sive libri xxx. Antverpiae.

Fiori, A. 1898. Ranùnculus (Tourn.) L. In A. Fiori \& G. Paoletti (Eds), Flora Analitica d'Italia 1: 500-516. Padova.

— 1924. Ranùnculus L. In A. Fiori, Nuova Flora Analitica d'ltalia 1: 659-678. Firenze.

Gandoger, M. 1889. Ranunculus. In Florae Europae 1: 167-169. Parisiis.

Gennari, P. 1866. Specie e varietà più rimarchevoli e nuove da aggiungere alla Flora Sarda: 29. Cagliari.

Greuter, W., Burdet, H.M. \& Long, G. 1989. Med-Checklist 4. Dicotyledones (Lauraceae-Rhamnaceae). Genève.

Halácsy E. von, 1904. Bericht der Sektion für Botanik. Versammlung am 29. April 1904. Verhandlungen der kaiserlich-königlichen zoologisch-botanischen Gesellschaft in Wien 54: 483-484.

Jahandiez, E. \& Maire, R. 1932. Catalogue des plantes du Maroc 2. Alger,

Jalas, J. \& Suominen, J. 1989. Atlas Florae Europaeae 8. Nymphaeaceae to Ranunculaceae. Helsinki.

Linnaeus, C. 1738. Hortus Cliffortianus. Amsterdam.

- 1753. Species plantarum. Holmiae [Stockholm].

López González, G. 1986. Ranunculus L. Sect. 6. Physoplyyllum Freyn. In S. Castroviejo et al. (Eds), Flora Iberica 1: 325-326. Madrid.

Maire, R. 1964. Ranunculus. In R. Maire, (Ed.), Flore de l'Afrique du Nord 11: I12204. Paris.

Meikle, R. D. 1977. Flora of Cyprus 1. Kew.

Morison, R. 1680. Plantarum historiae universalis Oxoniensis 2. Oxonii.

Nègre, R. 1961. Petite flore des régions arides du Maroc Occidental 1. Paris.

Pampanini, R. 1931. Prodromo della Flora Cirenaica. Forlì.

_ \& Zanon, V. 1919. Nuovi contributi alla conoscenza della flora della Cirenaica. Nuovo Giorn. Bot. Ital., n.s., 26: 205-220.

Paradis G., Pozzo di Borgo, M.-L. \& Jeanmonod, D. 2000. Ranunculus bullatus L. In D. Jeanmonod (Ed.), Notes et contributions à la flore de Corse, XV1. Candollea 55: 59 (fig. 2), 62.

Pereira Coutinho, A.X. 1974. Flora de Portugal, $2^{\text {nd }}$ ed. (Reprint). Leutershausen.

Pignatti, S. 1982. Flora d'Italia 1. Bologna.

Pottier-Alapetite, G. 1979. Flore de la Tunisie. Angiospermes-Dicotyledones. ApetalesDialypetales 1. Paris.

Qaiser, M. 1984. Ranunculaceae. In S.M.H. Jafri \& A. El-Gadi (Eds), Flora of Libya 108: 1-54. Tripoli.

Quézel, P. \& Santa, S. 1962. Nonvelle Flore de l'Algérie et des régions désertiques méridionales 1. Paris.

Rechinger K.H. fil. 1943. Flora Graeca. Wien.

Savage, S. 1945. A catalogue of the Linnaean herbarium. London.

Smythies, B.E. 1984. Flora of Spain and the Balearic Islands. Checklist of vascular plants. 1l. Cruciferae-Rutaceae. Englera 3: 213-486.

Turland, N.J., Chilton, L. \& Press, J.R. 1993. Flora of the Cretan area. Annotated checklist \& atlas. London.

_ \& Jarvis, C.E. (Eds) 1997. Typification of Linnaean specific and varietal names in the Leguminosae (Fabaceae). Taxon 46: 457-485.

Tutin, T.G. 1964. Ranunculus L. In T.G. Tutin et al. (Eds), Flora Europaea 1: 223-237. Cambridge.

\& Akeroyd, J.R. 1993. Ranunculus L. In T.G. Tutin et al. (Eds), Flora Europaea, $2^{\text {nd }}$ ed. 1: 269-285. Cambridge.

Vierhapper, F. \& Rechinger, K.H. fil. 1935. Bearbeitung der von lgnaz Dörfler im Jahre 1904 auf Kreta gesammelten Blüten- und Farnpflanzen. Österreichische Botanische Zeitschrift 84: 123-197.

Zangheri, P. 1976. Flora Ilalica 1. Padova. 



\title{
Catalogue of the lichens of Thailand
}

\author{
PATRICIA A. WOLSELEY \\ Department of Botany, The Natural History Museum, Cromwell Road, London SW7 5BD \\ BEGOÑA AGUIRRE-HUDSON \\ 58 Willowhayne Drive, Walton-on-Thames, Surrey KT12 2NR
}

PATRICK M. MCCARTHY

Australian Biological Resources Study, GPO Box 787, Canberra, A.C.T. 2601, Australia

\begin{abstract}
SYNOPSIS. A catalogue of the lichens of Thailand is provided based on published reports. It includes publication details of 554 species and infra-specific taxa together with information on distribution in Thailand, occurrence in other south and south-east Asian countries, literature references and the locations of named specimens.
\end{abstract}

\section{INTRODUCTION}

Before the 1990s, knowledge of the diversity and distribution of the lichen flora of Thailand was based almost entirely on collections made by a handful of foreign visitors in the first half of the twentieth century (Wolseley \& Aguirre-Hudson, 1991). During a research project on lichens as environmental indicators based at the Natural History Museum, London, a comprehensive survey of literature concerning lichens in south-east Asia was made and a data base constructed, which forms the basis of this checklist (Aguirre-Hudson \& Wolseley, 1994). It was not possible during this time to trace all types described in the literature, or to confirm the accuracy of many earlier identifications. Since then there has been a rapid and productive expansion of lichenological research by Thai practitioners, especially at the Lichen Research Unit, Ramkhamhaeng University, Bangkok, further expanding the list and including many newly described species (Boonpragob et al., 1998; Pooprang et al., 1999; Vongshewarat et al., 1999; Homchantara \& Coppins, 2002). The present checklist is conceived as a working document for all researchers in south-east Asia to provide both the literature sources and herbaria in which specimens from Thailand may be seen.

\section{SCOPE AND ARRANGEMENT}

This catalogue documents all 554 currently accepted taxa that have been reported in the literature from Thailand, as well as synonyms previously applied to Thai specimens. Genera and species are arranged in alphabetical order, and generic and family concepts follow Hawksworth et al. (1995). Where new combinations have not been made, taxa have been placed in the current genus within square brackets []. Accepted names are shown in bold and synonyms in italics. Distribution within Thailand is given where possible by province including new provinces and their former names (Fig. 1). Literature pertaining to distribution in Thailand is cited and references provided. Location of specimens in herbaria is indicated by abbreviations following Index Herbariorum (Holmgren et al., 1990) as shown below:

AAU: Herbarium Jutlandicum, Botanical Institute, University of Aarhus, Denmark.
BKF: Forest Herbarium, Royal Forest Department, Bangkok, Thailand.

BM: Herbarium, Botany Department, The Natural History Museum, London, UK.

C: Herbarium, Botanical Museum, University of Copenhagen, Denmark.

CANB: Australian National Herbarium, CSIRO, Canberra, Australia.

CMU: Herbarium, Biology Department, Chiang Mai University, Thailand.

COLO: Herbarium, Colorado University, Colorado, USA.

DUKE: Hebarium, Botany Department, Duke University, Durham, North Carolina, USA.

E: Herbarium, Royal Botanic Garden, Edinburgh, UK.

FH: Farlow Refence Library and Herbarium of Cryptogamic Botany, Harvard University, Cambridge, Massachusetts, USA.

GB: Herbarium, Botanical Museum, Gothenburg, Sweden.

H: Herbarium, Botanical Museum, University of Helsinki, Finland.

KF: Herbarium, Forest Research Institute, Seoul, Korea.

KYO: Herbarium, Botany Department, Kyoto University, Japan. L: $\quad$ Rijksherbarium, Leiden, Netherlands.

LG: Herbarium, Botany Department, Liège University, Belgium. OSA: Herbarium, Osaka Museum of Natural History, Japan.

PSU: Herbarium, Department of Biology, Prince of Songkla University, Haad Yai, Thailand.

RAMK: Herbarium, Biology Department, Ramkhamhaeng University, Bangkok, Thailand.

TNS: Herbarium, Department of Botany, National Science Museum, Tokyo, Japan.

TUR-V: Vainio herbarium, Biology Department, University of Turku, Finland.

UPS: Botanical Museum, Uppsala University, Sweden.

US: United States National Herbarium, Botany Department, Smithsonian Institution, Washington DC, USA.

Distribution of taxa in in other countries in adjacent areas of south and south-east Asia is indicated under the subheading 'AsIA'. We have avoided making taxonomic judgements on specimens we have not examined. Consequently, a number of highly dubious identifications remain, as do species which will almost certainly be transferred to other genera. 


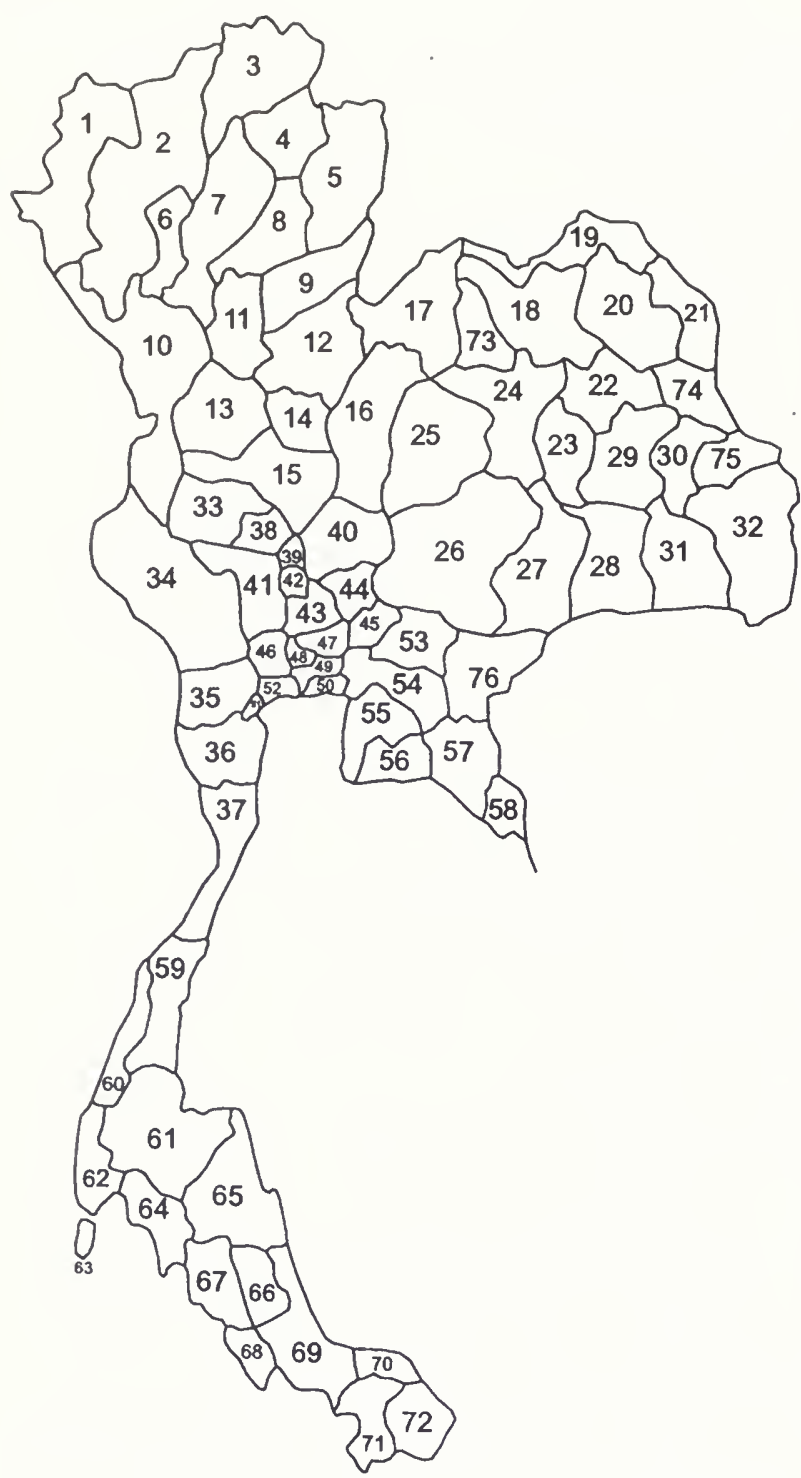

Key to provinees of Thailand

Northern region

1. Mae Hong Son

2. Chiang Mai

3. Chiang Rai

4. Phayao

5. Nan

6. Lamphun

7. Lampang

8. Phrae

9. Uttardit

10. Tak

11. Sukhotai

12. Phitsanulok

13. Kamphaeng Phet

14. Phichit

15. Nakhon Sawan

Northeastern region

16. Petchabun

17. Loei

18. Udon Thani

19. Nong Khai

20. Sakon Nakhon

21. Nakhon Phanom

22. Kalasin

23. Maha Sarakham

24. Khon Kaen

Eastern region

25. Chaiyaphum

26. Nakhon Ratchasima

27. Buri Ram

28. Surin

29. Roi Et

30. Yasothon

31. Si Sa Ket

32. Ubon Ratchathani

Southwestern region

33. Uthai Thani

34. Kanchanaburi

35. Ratchaburi

36. Phetchaburi

37. Prachuap Khiri Khan

Central region

38. Chai Nat
39. Sing Buri

40. Lop Buri

41. Suphan Buri

42. Ang Thong

43. Phra Nakhon Si Ayutthaya

44. Saraburi

45. Nakhon Nayok

46. Nakhon Pathom

47. Pathum Thani

48. Nonthaburi

49. Bangkok

50. Samut Prakan

51. Samut Songkram

52. Samut Sakhon

Southeastern region

53. Prachin Buri

54. Chachoengsao

55. Chon Buri

56. Rayong

57. Chanthaburi

58. Trat

Peninsular region

59. Chumphon

60. Ranong

61. Surat Thani

62. Phangnga

63. Phuket

64. Krabi

65. Nakhon Si Thammarat

66. Phatthalung

67. Trang

68. Satun

69. Songkhla

70. Pattani

71. Yala

72. Narathiwat

New provinees

73. Nong Bua Lamphu formerly in Udon Thani

74. Mukdahan formerly in Nakhon Phanom

75. Amnat Charoen formerly in Ubon Ratchathani

76. Sra Kaeo formerly in Prachin Buri

Fig. 1 Map of Thailand showing location of provinces. Reproduced with permission from Hamalainen \& Pinratana (2001).

Although this Catalogue is based exclusively on published reports and Homchantara's unpublished thesis, we have not included data from an anonymous checklist on the web-site of the Lichen Research Unit at Ramkhamhaeng University, Bangkok (www.ru.ac.th/lichen). This checklist includes the names (without additional information) of 404 species based on specimens held at RAMK. However, although many names can be attributed to literature sources and authoritative determinations, a significant proportion are unauthenticated and are previously unpublished taxa which must be considered possibly doubtful. We are aware that this Catalogue documents a rather small portion of what is almost certainly a very diverse lichen flora; indeed, the actual number of species is probably in excess of 2000 . However, we hope that it will provide a basis for future taxonomic research on Thai lichens, and that the research programs currently underway in Thailand will ensure its rapid obsolescence.

\section{CATALOGUE}

Anisomeridium (Müll. Arg.) M. Choisy; Monoblastiaceae

\section{[Anisomeridium] Microthelia asiaticum Vain., Hedwigia} 46: 180 (1907).

Thailand. Trat (Koh Chang I.). Vainio (1909: 149), Hawksworth (1985: 135).

SPECIMEN. TUR-V (holotype).

AsIA. India.

Anisomeridium foliicola R. Sant. \& Tibell, Austrobaileya 2: 531 (1988).

THAILAND. Nakhon Ratchasima (Khao Yai N.P.). Boonpragob et al. (1998: 216). 
SPECIMENS. CANB.

ASIA. India, Papua New Guinea, Philippines, Sri Lanka.

Anthracothecium Hampe ex A. Massal.; Pyrenulaceae

Anthracothecium macrosporum Müll. Arg., Linnaea 63: 44 (1888).

Anthracothecium pyrenuloides auct. non (Mont.) Müll. Arg.

Thailand. Chumphon (Koh Tao I.). Paulson (1930: 99, as Anthracothecium pyrenuloides).

SPECIMENS. BM.

ASIA. India, Indonesia.

Anthracothecium parameroides (Vain.) Zahlbr., Cat. Lich. Univ. 1: 466 (1922).

Bottaria parameroides Vain.

ThaILAND. Trat (Koh Chang I.). Vainio (1907: 179; 1909: 143, both as Bottaria parameroides).

SPECIMEN. TUR-V (holotype).

ASIA. Indonesia.

Anthracothecium prasinum (Eschw.) R.C. Harris, in Egan, Bryologist 90: 163 (1987).

ThAILAND. Nakhon Ratchasima (Khao Yai N.P.), Uthai Thani (Huay Kha Khaeng Wildlife Sanctuary). Wolseley \& AguirreHudson (1997a: 333), Boonpragob et al. (1998: 216).

SPECIMENS. BM, RAMK.

AsIA. Papua New Guinea.

Anthracothecium roseum (Vain.) Zahlbr., Cat. Lich. Univ. 1: 468 (1922).

Bottaria rosea Vain.

ThaIlAnd. Trat (Koh Chang I.). Vainio (1907: 179; 1909: 143, both as Bottaria rosea).

SPECIMEN. TUR-V (holotype).

ASIA. -

Arthonia Ach.; Arthoniaceae

Arthonia complanata Fée, Essai Crypt. Écorc. Offic.: 54 (1824).

ThaILAND. Trat (Koh Chang I.). Vainio (1909: 139).

SPECIMEN. TUR-V.

ASIA. China, Indonesia, Papua New Guinea, Philippines.

Arthonia aff. mira R. Sant., Symb. Bot. Upsal. 12(1): 77 (1952).

THAILAND. Nakhon Ratchasima (Khao Yai N.P.). Boonpragob et al. (1998: 216),

SPECIMENS. RAMK.

ASIA. -
Arthonia obscuratula Zahlbr., Cat. Lich. Univ. 2: 71

(1922).

Arthonia stenographella subsp. obscurata Vain.

ThAILAND. Trat (Koh Chang I.). Vainio (1909: 140, as Arthonia stenographella subsp. obscurata).

SPECIMEN. TUR-V (holotype).

ASIA. -

Arthonia ochrodes Nyl., in Willey, Syn. Gen. Arthonia: 10 (1890).

ThaILAND. Trat (Koh Chang I.). Vainio (1909: 142).

SPECIMEN. TUR-V.

ASIA. -

Arthonia perpallens Nyl., Ann. Sci. Nat., Bot., sér. 4, 20: 231 (1863).

ThaILAND. Trat (Koh Chang I.). Vainio (1909: 141).

SPECIMENS. TUR-V.

ASIA. -

Arthonia rhizophorae Vain., Bot. Tidsskr. 29: 141 (1909).

ThAILAND. Trat (Koh Chang I.). Vainio (1909: 141).

SPECIMEN. TUR-V (holotype).

ASIA. -

Arthonia varia Nyl. var. stenographella (Nyl.) Vain., Ann. Acad. Sci. Fenn., ser. A, 15(6): 393 (1921).

Arthonia stenographella Nyl.

ThaILAND. Trat (Koh Chang I.). Vainio (1909: 140, as $A$. stenographella).

SPECIMEN. TUR-V (holotype).

ASIA. Philippines, Sri Lanka.

Arthopyrenia A. Massal.; Arthopyreniaceae

Arthopyrenia siamensis Vain., Suom. Elain-ja Kasvit. Seuran Van. Julk. 1(3): 55 (1921).

ThaIland. Chiang Mai (Doi Suthep N.P.). Vainio (1921: 55).

SPECIMEN. TUR-V (holotype).

ASIA. -

Arthothelium A. Massal.; Arthoniaceae

Arthothelium monosporum (Vain.) Zahlbr., Cat. Lich. Univ. 2: 129 (1922).

Arthonia monospora Vain.

THAILAND. Chiang Mai (Doi Suthep N.P.). Vainio (1921: 54, as Arthonia monospora).

SPECIMEN. TUR-V (holotype).

ASIA. - 
Arthothelium ruanum (A. Massal.) Körb., Parerg. Lich.: 263 (1861).

Arthonia ruana A. Massal.

Thalland. Trat (Koh Chang I.). Vainio (1909: 139, as Arthonia rllana).

SPECIMEN. TUR-V.

ASIA. -

Arthothelium ruanum var. phaeosporum (Vain.) Zahlbr., Cat. Lich. Univ. 2: 132 (1922).

Arthonia ruana var. phaeospora Vain.

ThaIlAND. Trat (Koh Chang I.). Vainio (1909: 139, as Arthonia ruana var. phaeospora).

SPECIMEN. TUR-V (holotype).

ASIA. -

Arthothelium spectabile Flotow ex A. Massal., Ric. Auton. Lich. Crost.: 54 (1852).

ThaIland. Chumphon (Koh Tao I.). Paulson (1930: 99).

SPECIMEN. BM.

ASIA. China, India, Malaysia, Sri Lanka.

Bacidia De Not.; Bacidiaceae

Bacidia affinis Vain., Acta Soc. Fauna Fl. Fenn. 53(1): $146 \& 154$ (1922).

Bacidia atrosanguinea Th. Fr.

ThaIland. Chumphon (Koh Tao I.). Paulson (1930:99, as Bacidia atrosanguinea).

SPECIMEN. BM (not located).

ASIA. -

Bacidia manilensis var. siamensis Vain., Suoml. Elain-ja Kasvit. Seuran Van. Julk. 1(3): 46 (1921).

Thailand. Chiang Mai (Doi Suthep N.P.). Vainio (1921: 46).

SPECIMEN. TUR-V (holotype).

ASIA. -

Bacidia umbrina var. turgida Th. Fr., Lichenogr. Scand. 1: 365 (1874).

ThaIland. Chumphon (Koh Tao I.). Paulson (1930: 99).

SPECIMEN. BM (not located).

ASIA. -

Bathelium Ach.; Trypetheliaceae

Bathelium madreporiforme (Eschw.) Trevis., Spighe e Paglie: 20 (1853).

Laurera madreporiformis (Eschw.) Riddle.

THAILAND. Nakhon Ratchasima (Khao Yai N.P.). Boonpragob et al. (1998: 217, as Laurera madreporiformis).
SPECIMENS. CANB.

ASIA. India, Myanmar.

Brigantiaea Trevis.; Brigantiaeaceae

Brigantiaea leucoxantha (Spreng.) R. Sant \& Hafellner, in Hafellner \& Bellemère, Nova Hedwigia 35: 246 (1981).

ThaIland. Chiang Mai (Doi Suthep N.P.), Nakhon Ratchasima (Khao Yai N.P.), Uthai Thani (Huay Kha Khaeng Wildlife Sanctuary). Wolseley \& Aguirre-Hudson (1997a: 333), Boonpragob et al. (1998: 216).

SPECIMENS. BM, RAMK.

ASIA. India, Malaysia, Papua New Guinea, Philippines, Sri Lanka, Taiwan.

Brigantiaea tricolor (Mont.) Trevis., Spighe e Paglie: 9 (1853).

ThaIlAnd. Chiang Mai (Doi Suthep N.P., Chiang Dao N.P.), Lampang (Doi Khun Tan N.P.). Wolseley \& Aguirre-Hudson (1997a: 333).

SPECIMENS. BM, CMU.

ASIA. India, Indonesia, Malaysia, Papua New Guinea, Sri Lanka, Taiwan.

Buellia De Not.; Physciaceae

Buellia blastenioides Vain., Hedwigia 46: 171 (1907).

Thailand. Trat (Koh Chang I.). Vainio (1909: 114).

SPECIMEN. TUR-V (holotype).

ASIA. -

Buellia pleiophoroides var. meiospermoides Vain., Bot. Tidsskr. 29: 113 (1909).

ThAiland. Chiang Mai (Doi Suthep N.P.), Trat (Koh Chang I.), Uthai Thani (Huay Kha Khaeng N.P.). Vainio (1909: 113), Wolseley \& Aguirre-Hudson (1997a: 339).

SPECIMENS. BM, TUR-V (holotype).

ASIA. -

Buellia stramineoatra Vain., Hedwigia 46: 172 (1907).

Thailand. Trat (Koh Chang I.). Vainio (1909: 115).

SPECIMENS. TUR-V (syntypes).

ASIA. -

Buellia subdives Vain., Hedwigia 46:172 (1907).

ThAILAND. Trat (Koh Chang I.).Vainio (1907: 172; 1909: 114).

SPECIMENS. TUR-V (syntypes).

ASIA. -

Bulbothrix Hale; Parmeliaceae

Bulbothrix goebelii (Zenker) Hale, Smithsonian Contr. Bot. 32: 14 (1976). 
Parmelia addenda Vain.

ThaIland. Chiang Mai (Doi Suthep N.P., Doi Inthanon N.P., Queen Sirikit Botanical Garden), Trat (Koh Chang I.), Uthai Thani (Huay Kha Khaeng Wildlife Sanctuary). Vainio (1909: 107, as Parmelia addenda), Hale (1976a: 14), Mongkonsuk et al. (1996: 16), Wolseley \& Aguirre-Hudson (1997a: 333).

SPECIMENS. C(lectotype), TUR-V (isolectotype), BKF, BM, CMU, US, Queen Sirikit Botanical Garden Herbarium.

AsIA. India, Indonesia, Malaysia, Papua New Guinea, Philippines, Taiwan.

Bulbothrix hypochraea (Vain.) Hale, Phytologia 28: 480 (1974).

ThaIland. Chiang Mai (Queen Sirikit Botanical Garden). Pooprang et al. (1999: 118).

SPECIMENS. RAMK.

ASIA. -

Bulbothrix isidiza (Nyl.) Hale, Phytologia 28: 480 (1974).

ThAILAND. Chiang Mai (Doi Suthep N.P., Doi Inthanon N.P., Chiang Dao N.P., Maetang), Lampang (Doi Pae Luang), Loei (Ban Sui, Phu Kradung), Phitsanulok (Phu Hin Rongkla, N.P.), Uthai Thani (Huay Kha Khaeng Wildlife Sanctuary). Wolseley \& AguirreHudson (1995: 318; 1997a: 333; 1997b: 355-356), Moon et al. (2000a: 99).

\section{SPECIMENS. BKF, BM, CMU.}

ASIA. China, India, Indonesia, Malaysia, Papua New Guinea, Philippines, Sri Lanka, Taiwan.

Bulbothrix laevigatula (Nyl.) Hale, Phytologia 28: 480 (1974).

Parmelia hookeri Taylor.

Thalland. Chiang Mai (Doi Suthep). Vainio (1921: 37, as Parmelia hookeri).

SPECIMEN. TUR-V.

ASIA. -

Bulbothrix meizospora (Nyl.) Hale, Phytologia 28: 480 (1974).

ThaILAND. Chiang Mai (Queen Sirikit Botanical Garden), Loei (Phu Rhoa N.P.), Nakhon Nayok (Khao Yai N.P.). Boonpragob et al. (1998: 216), Pooprang et al. (1999: 118).

SPECIMENS. BM, RAMK.

AsIA. India, Indonesia, Nepal, Sri Lanka.

Bulbothrix pigmentacea (Hale) Hale, Phytologia 28: 480 (1974).

ThaILAND. Chiang Mai (Doi Suthep N.P., Doi Inthanon N.P.), Uthai Thani (Huay Kha Khaeng Wildlife Sanctuary). Wolseley \& Aguirre-Hudson (1995: 318; 1997a: 333; 1997b: 355-56).

SPECIMENS. BKF, BM, CMU.

ASIA. Malaysia, Philippines.
Bulbothrix queenslandica (Elix \& G.N. Stevens) Elix, Mycotaxon 47: 126 (1993).

Thalland. Chiang Mai (Queen Sirikit Botanical Garden), Loei (Nahaew N.P.), Phitsanulok (Phu Hin Rongkla N.P.). Pooprang et al. (1999: 119).

SPECIMENS. RAMK.

ASIA. -

Bulbothrix sensibilis (J. Steiner \& Zahlbr.) Hale, Phytologia 28: 481 (1974).

ThaIlAnd. Loei (Nahaew N.P.). Pooprang et al. ( 1999: 119). SPECIMENS. RAMK.

ASIA. India, Sri Lanka.

Bulbothrix setschawensis (Zahlbr.) Hale, Phytologia 28: 481 (1974).

ThaILAND. Chiang Mai (Doi Inthanon N.P.), Lampang, Uthai Thani (Huay Kha Khaeng Wildlife Sanctuary). Wolseley \& AguirreHudson (1997a: 333).

SPECIMENS. BKF, BM, CMU.

AsIA. China, India, Nepal.

\section{Byssoloma Trevis.; Pilocarpaceae}

Byssoloma chlorinum (Vain.) Zahlbr., Cat. Lich. Univ. 8: 233 (1932).

ThaILAND. Nakhon Ratchasima (Khao Yai N.P.). Boonpragob et al. (1998: 216).

SPECIMENS. RAMK.

ASIA. India, Indonesia, Papua New Guinea.

Byssoloma leucoblepharum (Nyl.) Vain., Dansk Bot. Ark. 4: 23 (1926).

ThAILAND. Nakhon Ratchasima (Khao Yai N.P.). Wolseley \& Aguirre-Hudson (1997a: 333), Boonpragob et al. (1998: 216).

SPECIMENS. BM, RAMK.

ASIA. India, Indonesia, Malaysia, Papua New Guinea, Philippines, Singapore, Vietnam.

Byssoloma tricholomum (Mont.) R. Sant., Symb. Bot. Upsal. 12(1): 480 (1952).

THAILAND. Nakhon Ratchasima (Khao Yai N.P.). Boonpragob et al. (1998: 216).

SPECIMENS. RAMK.

ASIA. China, Philippines, Singapore.

Calenia Müll. Arg. emend. R. Sant.; Gomphillaceae

Calenia graphidea Vain., Ann. Acad. Sci. Fenn., ser. A, 15: 163 (1921).

THAILAND. Nakhon Ratchasima (Khao Yai N.P.). Boonpragob et al. (1998: 216).

SPECIMENS. RAMK. 
AsIA. Indonesia, Malaysia, Papua New Guinea, Philippines.

Calenia aff. phyllogena (Müll. Arg.) R. Sant., Symb. Bot. Upsal. 12(1): 349 (1952).

THAILAND. Nakhon Ratchasima (Khao Yai N.P.). Boonpragob et al. (1998: 216).

\section{SPECIMENS. RAMK.}

ASIA. India, Papua New Guinea, Philippines, Sri Lanka.

Calopadia Veězda; Ectolechiaceae

Calopadia fusca (Müll. Arg.) Veĕzda, Folia Geobot. Plytotax. 21: 215 (1986).

ThAILAND. Nakhon Ratchasima (Khao Yai N.P.). Boonpragob et al. (1998: 216).

SPECIMENS. CANB.

ASIA. Indonesia, Papua New Guinea, Philippines, Sri Lanka.

Caloplaca Th. Fr.; Teloschistaceae

Caloplaca aequata (Hue) Zahlbr., Cat. Lich. Univ. 7: 211 (1931).

Thailand. Chumphon (Koh Tao I.). Paulson (1930: 99).

SPECIMEN. BM (not located).

AsIA. -

Caloplaca flavorubescens (Huds.) Laundon, Lichenologist 8: 147 (1976).

Caloplaca aurantiaca (Lightf.) Th. Fr.

THAILAND. Chiang Mai (Doi Inthanon N.P.), Chumphon (Koh Tao I.). Paulson (1930: 99, as Caloplaca aurantiaca).

SPECIMENS. BM.

ASIA. Sri Lanka, Taiwan.

Caloplaca holocarpa (Hoffm.) A.E. Wade, Lichenologist 3: 11 (1965).

Caloplaca pyracea (Ach.) Th. Fr.

Thailand. Chumphon (Koh Tao I.). Paulson (1930: 99, as Caloplaca pyracea).

SPECIMEN. BM (not located).

ASIA. China, India.

[Caloplaca] Placodium testaceorufum Vain., Hedwigia 46: 170 (1907).

Thailand. Trat (Lem Ngob). Vainio (1909: 111, as Placodium testaceorufum).

SPECIMEN. TUR-V (holotype).

ASIA. -

Canomaculina Elix \& Hale; Parmeliaceae

Canomaculina subsumpta (Nyl.) Elix, Mycotaxon 65: 477 (1997).
Rimeliella subsumpta (Nyl.) Kurok.

Thailand. Chiang Mai (Maetang). Kurokawa (1991: 9, as Rimeliella subsumpta); Moon et al. (2000a: 99).

SPECIMEN. TNS.

ASIA. India, Papua New Guinea.

Canomaculina subtinctoria (Zahlbr.) Elix, Mycotaxon 65: 477 (1997)

ThaILAND. Chiang Mai (Queen Sirikit Botanical Garden), Loei (Nahaew N.P.). Mongkonsuk et al. (1996: 17), Pooprang et al. (1999: 119).

SPECIMENS. RAMK.

AsIA. China, India, Indonesia, Nepal.

Canoparmelia Elix \& Hale; Parmeliaceae

Canoparmelia adspersa (Vain.) Elix \& Hale, in Elix, Johnston \& Verdon, Mycotaxon 27: 278 (1986).

Parmelia adspersa Vain.

Thailand. Trat (Lem Ngob), Vainio (1907: 168, as Parmelia adspersa).

SPECIMENS. C (isolectotype), TUR-V (lectotype and syntype).

AsIA. Papua New Guinea, Philippines.

Canoparmelia concrescens (Vain.) Elix \& Hale, Mycotaxon 27: 278 (1986).

Thailand. Phitsanulok (Phu Hin Rongkla, N.P.). Moon et al. (2000a: 100).

SPECIMENS. TNS.

ASIA. -

Canoparmelia ecaperata (Müll. Arg.) Elix \& Hale, in Elix, Johnston \& Verdon, Mycotaxon 27: 278 (1986).

Parmelia malaccensis var. laeteflavens Vain.

ThaIland. Chiang Mai (Doi Suthep N.P., Doi Inthanon N.P., Chiang Dao N.P., Maetang), Loei (Phu Kradung N.P.), Nakhon Ratchasima (Khao Yai N.P.), Phitsanulok (Phu Hin Rongkla, N.P.), Uthai Thani (Huay Kha Khaeng Wildlife Sanctuary). Vainio (1921: 38, as Parmelia malaccensis var laeteflavens), Wolseley \& AguirreHudson (1995: 318; 1997a: 333; 1997b: 355-56), Boonpragob et al. (1998: 216), Moon et al. (2000a: 100).

SPECIMENS. BM, CMU, RAMK, TNS, TUR-V (lectotype and syntype of $P$. malaccensis var laeteflavens), US.

ASIA. India, Nepal.

Canoparmelia owariensis (Asahina) Elix, Mycotaxon 47: 127 (1993).

Pseudoparmelia owariensis (Asahina) Hale.

Thailand. Chiang Mai (Doi Saget), Loei (Ban Sui, Phu Kradung), Phitsanulok (Phu Hin Rongkla, N.P.). Hale (1976b: 39, as Pseudoparmelia owariensis), Moon et al. (2000a: 100).

SPECIMENS. TNS.

ASIA. China (Hong Kong). 
Canoparmelia pustulescens (Kurok.) Elix, Mycotaxon 47: 127 (1993).

ThaIland. Chiang Mai (Doi Saget), Loei (Phu Kradung). Moon et al. (2000a: 101).

SPECIMENS. TNS.

AsIA. India.

Canoparmelia salacinifera (Hale) Elix \& Hale, in Elix, Johnston \& Verdon, Mycotaxon 27: 279 (1986).

Pseudoparmelia salacinifera (Hale) Hale.

ThaILAND. Chiang Mai (Doi Saget, Doi Suthep N.P.), Loei (Phu Kradung N.P.). Hale (1976a: 46, as Pseudoparmelia salacinifera), Wolseley \& Aguirre-Hudson (1995: 318), Moon et al. (2000a: 101).

SPECIMENS. BKF, BM, CMU, TNS, US.

AsIA. -

Canoparmelia texana (Tuck.) Elix \& Hale, in Elix, Johnston \& Verdon, Mycotaxon 27: 279 (1986).

Thailand. Chiang Mai (Chiang Dao N.P., Queen Sirikit Botanical Garden), Lampang (Doi Khun Tan N.P.), Loei (Phu Kradung N.P.). Hale (1976a: 53), Moon et al. (2000a: 101).

SPECIMENS. BKF, BM, CMU, TNS.

ASIA. India, Malaysia, Sri Lanka.

Catillaria A. Massal.; Catillariaceae

Catillaria testaceolivens (Vain.) Zahlbr., Cat. Lich. Univ. 4: 79 (1927).

Lecidea testaceolivens Vain.

THAILAND. Trat (Koh Chang I.). Vainio (1907: 173; 1909: 117, both as Lecidea testaceolivens).

SPECIMEN. TUR-V (holotype).

AsIA. -

Catillaria unicolor (Vain.) Zahlbr., Cat. Lich. Univ. 4: 83 (1927).

Lecidea unicolor Vain.

Thailand. Trat (Koh Chang 1.). Vainio (1907: 173; 1909: 118, both as Lecidea unicolor).

SPECIMEN. TUR-V (holotype).

ASIA. -

Cetrariopsis Kurok.; Parmeliaceae

Cetrariopsis wallichiana (Taylor) Kurok., Mem. Nat. Sci. Mus., Tokyo 13: 140 (1980).

Cetraria wallichiana (Taylor) Müll. Arg.

Thalland. Chiang Mai (Doi Suthep). Vainio (1921: 36, as Cetraria wallichiana), Kurokawa (1980: 140), Wolseley \& Aguirre-Hudson (1995: 318; 1997a: 333).

SPECIMENS. BM, PSU, TNS.
ASIA. China, Indonesia, Nepal, Philippines, Taiwan.

Cetrelia W.L. Culb.\& C.F. Culb.; Parmeliaceae

Cetrelia braunsiana (Müll. Arg.) W.L. Culb. \& C.F. Culb., Contr. U.S. Natl. Herb. 34: 493 (1968).

Cetraria collata f. isidiata Asahina.

Thailand. Chiang Mai (Doi Inthanon N.P.). Sato (1962: 3, as Cetraria collata f. isidiata), Wolseley \& Aguirre-Hudson (1997a: $333,337)$.

SPECIMENS. BM, TNS.

AsIA. China, India, Malaysia, Papua New Guinea, Philippines, Taiwan.

Cetrelia japonica (Zahlbr.) W.L. Culb. \& C.F. Culb., Contr. U.S. Natl. Herb. 34: 511 (1968).

ThaIland. Chiang Mai (Doi Inthanon N.P.), Nakhon Nayok (Khao Yai N.P.). Wolseley \& Aguirre-Hudson (1997a: 333), Boonpragob et al. (1998: 216).

SPECIMENS. BM, RAMK.

ASIA. Indonesia, Malaysia, Taiwan.

Cetrelia nuda (Hue) W.L. Culb. \& C.F. Culb., Contr. U.S. Natl. Herb. 34: 513 (1968).

THAILAND. Chiang Mai (Doi Inthanon N.P.). Wolseley \& AguirreHudson (1997a: 337, 339).

SPECIMEN. BM.

ASIA. China, Taiwan.

Cetrelia olivetorum (Nyl.) W.L. Culb. \& C.F. Culb, Contr. U.S. Natl. Herb. 34: 515 (1968).

ThaILAND. Chiang Mai (Doi Inthanon N.P.). Wolseley \& AguirreHudson (1997a: 337, 339).

SPECIMEN. BM.

AsIA. China, India, Papua New Guinea, Taiwan.

Chaenotheca (Th. Fr.) Th. Fr.; Coniocybaceae

Chaenotheca trichialis (Ach.) Th. Fr., Nova Acta Regiae Soc. Sci. Upsal., ser. 3, 3: 251 (1860).

THAILAND. Nakhon Ratchasima (Khao Yai N.P.). Boonpragob et al. (1998: 216).

SPECIMEN. RAMK.

ASIA. India.

Chiodecton Ach.; Roccellaceae

Chiodecton congestulum Nyl., Bull. Soc. Linn.

Normandie, sér. 2, 2: 106 (1868).

Thailand. Trat (Koh Chang I.). Thor (1990: 41).

SPECIMENS. C, TUR-V.

AsIA. India, Indonesia, Malaysia, Papua New Guinea, Taiwan. 
Chiodecton subsphaerale Nyl., Bull. Soc. Linn.

Normandie, sér. 2, 2: 106 (1868).

Thailand. Trat (Koh Chang I.). Vainio (1909: 138).

SPECIMENS. TUR-V.

ASIA. Malaysia.

Chroodiscus Müll. Arg.; Thelotremataceae

Chroodiscus mirificus (Kremp.) R. Sant., Symb. Bot.

Upsal. 12(1): 311 (1952).

Thailand. Nakhon Ratchasima (Khao Yai N.P.). Boonpragob et al. (1998: 216).

SPECIMENS. RAMK.

AsIA. India. Indonesia, Malaysia, Papua New Guinea, Philippines, Sri Lanka, Vietnam.

Chrysothrix Mont.; Chrysothricaceae

Chrysothrix candelaris (L.) Laundon, Lichenologist 13: $110(1981)$

Lepraria xanthina Vain.

Thalland. Chiang Mai (Doi Suthep N.P., Doi Inthanon N.P.), Trat (Koh Chang I.), Uthai Thani (Huay Kha Khaeng Wildlife Sanctuary). Vainio (1909: 151, as Lepraria xanthina), Wolseley \& Aguirre-Hudson (1997b: 355).

SPECIMENS. BM, TUR-V.

AsIA. China (Hong Kong), India, Philippines, Sri Lanka.

Cladia Nyl; Cladoniaceae

Cladia aggregata (Sw.) Nyl., Compt. Rend. Hebd. Séances Acad. Sci. 83: 8 (1876).

ThaIland. Loei (Phu Miang, Phu Kradung N.P.). Yoshimura (1978: 37).

SPECIMENS. AAU, C.

AsIA. China (Hong Kong), India, Indonesia, Malaysia, Nepal, Papua New Guinea, Philippines, Taiwan.

Cladonia P. Browne; Cladoniaceae

Cladonia calyciformis Nuno, J. Jap. Bot. 47: 161 (1972).

Thailand. Loei ( Phu Kradung N.P.). Yoshimura (1978: 37).

SPECIMENS. C, TNS (holotype).

ASIA.

Cladonia ciliata var. tenuis (Flörke) Ahti, Ann. Bot. Fenn. 15: 8 (1978).

Cladonia tenuis (Flörke) Harm.

Thailand. Phu Mieng. Yoshimura (1978: 37, as Cladonia tenuis). SPECIMENS. AAU, C.

ASIA. China, India.
Cladonia didyma var. vulcanica (Zoll. \& Moritzi) Vain., Acta Soc. Fauna Fl. Fenn. 4: 145 (1887).

Cladonia vulcanica Zoll.

ThaIland. Chiang Mai (Doi Inthanon N.P.), Loei (Phu Kradung N.P.). des Abbayes (1956: 259, as Cladonia vulcanica), Yoshimura (1978: 38, as Cladonia vulcanica).

SPECIMENS. TNS.

AsIA. India, Indonesia, Papua New Guinea, Philippines, Sri Lanka, Taiwan.

Cladonia furcata (Huds.) Schrad., Spicil. Fl. Germ.: 107 (1794).

THAILAND. Nakhon Ratchasima (Khao Yai N.P.). Boonpragob et al. (1998: 216).

SPECIMENS. RAMK.

AsIA. China, India, Indonesia, Taiwan.

Cladonia gymnopoda Vain., Acta Soc. Fauna Fl. Fenn. 10: 172 (1894).

Thailand. Loei (Phu Miang). Yoshimura (1978: 38).

SPECIMENS. AAU, C.

AsıA. India, Indonesia, Malaysia, Papua New Guinea, Philippines, Taiwan.

Cladonia modesta Ahti \& Krog, Ann. Bot. Fenn. 24: 88 (1987).

THAILAND. Nakhon Ratchasima (Khao Yai N.P.). Boonpragob et al. (1998: 216).

SPECIMENS. RAMK.

ASIA. -

Cladonia ramulosa (With.) Laundon, Lichenologist 6: 225 (1984).

Cladonia pityrea (Flörke) Fr.

Thailand. Chiang Mai (Doi Suthep N.P.), Khu Kawng, Loei (Phu Luang N.P.). Vainio (1921: 46, as C. pityrea), Yoshimura (1978: 38, as C. pityrea).

SPECIMENS. C.

ASIA. China (incl. Hong Kong), India, Indonesia, Malaysia, Papua New Guinea, Philippines, Sri Lanka.

Cladonia rangiferina (L.) F.H. Wigg., Primit. Fl. Holsat. 90: 29 (1780).

Thalland. Loei (Phu Kradung N.P.). Yoshimura (1978: 37), Kurokawa \& Kashiwadani (1988: Lich. Rar. Crit. Exs. No. 60).

SPECIMENS. C, TNS.

ASIA. China, India, Taiwan.

Cladonia rappii A. Evans, Trans. Connecticut Acad. Arts 38: 297 (1952).

Cladonia calycantha Delise ex Nyl. 
Thailand. Chiang Mai (Doi Suthep N.P.), Loei (Phu Kradung N.P.). Yoshimura (1978: 37, as C. calycantha).

Specimens. AAU, BM, C.

ASIA. China, India, Indonesia, Malaysia, Papua New Guinea, Philippines, Taiwan.

Cladonia siamea des Abbayes, Kew Bull. 2: 262 (1956).

Thalland. Chiang Mai (Doi Inthanon N.P., Doi Suthep N.P.), Loei (Phu Kradung N.P.). Yoshimura (1978: 37), Huovinen \& Ahti (1986: 182), Stenroos (1986: 164), Kurokawa \& Kashiwadani (1988: Lich. Rar. Crit. Exs. Nos. 61, 62).

SPECIMENS. BM (holotype), C, H (isotype), RAMK, TNS.

AsIA. Indonesia, Papua New Guinea.

Cladonia siamea f. evoluta des Abbayes, Kew Bull. 2: 262 (1956).

Thailand. Loei (Phu Kradung N.P.). des Abbayes (1956: 262).

SPECIMENS. BKF, BM (holotype).

ASIA. -

Cladonia siamea f. pulvinata des Abbayes, Kew Bull. 2: 263 (1956).

Thailand. Loei (Phu Kradung N.P). des Abbayes (1956: 263).

SPECIMENS. BKF, BM (holotype).

ASIA.

Cladonia squamosa (Scop.) Hoffm., Deutschl. Fl. 2: 125 (1796).

THAILAND. Nakhon Ratchasima (Khao Yai N.P.). Boonpragob et al. (1998: 216).

SPECIMENS. RAMK.

AsIA. China, India, Indonesia, Papua New Guinea, Philippines, Taiwan.

Cladonia subpityrea Sandst., in Zahlbruckner, Ann. Naturhist. Mus. Wien 42: 61 (1928).

ThaIland. Loei (Dan Sai). Yoshimura (1978: 38).

SPECIMENS. C.

AsIA. Papua New Guinea, Philippines, Taiwan.

Cladonia transindica Ahti, Ann. Bot. Soc. Zool-Bot. Fenn. 'Vanamo' 32(1): 72 (1961).

THAILAND. locality not known. des Abbayes (1964), Ahti (1984: 44), Stenroos (1988: 212).

SPECIMEN. ?

ASIA. China, Indonesia, Malaysia, Vietnam.

Coccocarpia Pers.; Coccocarpiaceae

Coccocarpia dissecta Swinscow \& Krog, Norweg. J. Bot. 23: 254 (1976).

ThaILAND. Chiang Mai (Queen Sirikit Botanical Garden, Doi
Suthep N.P., Doi Inthanon N.P.), Khiri Khan, Uthai Thani (Huay Kha Khaeng Wildlife Sanctuary). Mongkonsuk et al. (1996: 16).

SPECIMENS. BM, Queen Sirikit Botanical Garden Herbarium.

ASIA. Malaysia, Papua New Guinea, Philippines.

Coccocarpia erythroxyli (Spreng.) Swinscow \& Krog, Norweg. J. Bot. 23: 256 (1976).

Coccocarpia molybdaea Pers.

Thalland. Chiang Mai (Doi Suthep N.P., Doi Inthanon N.P.), Nakhon Ratchasima (Khao Yai N.P.), Trat (Koh Chang 1.), Uthai Thani (Huay Kha Khaeng Wildlife Sanctuary). Paulson (1930: 99, as C. molybdaea), Arvidsson (1982: 62), Boonpragob et al. (1998: 216).

SPECIMENS. BM, GB, L, RAMK, TNS.

ASIA. China (incl. Hong Kong), India, Malaysia, Myanmar, Papua New Guinea, Philippines, Singapore, Sri Lanka, Taiwan.

Coccocarpia glaucina Kremp., Nuovo Giorn. Bot. Ital. 7: 14 (1875).

ThaILAND. Chiang Mai (Doi Suthep N.P.), Nakhon Nayok (Khao Yai N.P.). Boonpragob et al. (1998: 216).

SPECIMENS. BM, RAMK.

ASIA. Malaysia, Papua New Guinea, Philippines.

Coccocarpia palmicola (Spreng.) Arv. \& D.J. Galloway, Bot. Not. 132: 242 (1979).

ThaIland. Chiang Mai (Queen Sirikit Botanical Garden, Doi Suthep N.P., Doi Inthanon N.P., Chiang Dao N.P.), Nakhon Nayok (Khao Yai N.P.), Tak (Ton Krabakyai N.P.), Uthai Thani (Huay Kha Khaeng Wildlife Sanctuary). Arvidsson (1982: 76), Wolseley \& Aguirre-Hudson (1997a: 333; 1997b: 355), Mongkonsuk et al. (1996), Boonpragob et al. (1998: 216).

SPECIMENS. BM, L, RAMK, TNS, Queen Sirikit Botanical Garden Herbarium.

ASIA. China (incl. Hong Kong), India, Malaysia, Myanmar, Papua New Guinea, Philippines, Singapore, Sri Lanka, Taiwan.

Coccocarpia pellita (Ach.) Müll. Arg. emend. R. Sant., Symb. Bot. Upsal. 12(1): 420 (1952).

ThAILAND. Nakhon Nayok (Khao Yai N.P.), Uthai Thani (Huay Kha Khaeng Wildlife Sanctuary). Boonpragob et al. (1998: 216).

SPECIMENS. BM, RAMK.

AsIA. China (Hong Kong), India, Indonesia, Malaysia, Papua New Guinea, Philippines, Sri Lanka.

Collema F.H. Wigg.; Collemataceae

Collema coccophyllum Nyl., Syn. Meth. Lich. 1: 112 (1858).

Thailand. Chiang Mai (Doi Suthep). Degelius (1974: 69).

SPECIMEN. TNS.

ASIA. India. 
Collema coilocarpum (Müll. Arg.) Zahlbr., Cat. Lich.

Univ. 3: 34 (1924).

Thalland. Chiang Mai (Doi Inthanon N.P.), Nakhon Ratchasima (Khao Yai N.P.), Uthai Thani (Huay Kha Khaeng Wildlife Sanctuary). Wolseley \& Aguirre-Hudson (1997a: 333), Boonpragob et al. (1998: 217).

SPECIMENS. BM, RAMK.

AsıA. India, Papua New Guinea, Philippines, Sri Lanka.

Conotrema Tuck.; Stictidaceae

Conotrema lumbricoides Sipman, in Aptroot et al., Biblioth. Lichenol. 64: 52 (1997).

ThaIlAnd. Nakhon Sri Thammarat (Khao Luang N.P.). Vongshewarat et al. (1999: 227)

SPECIMENS. RAMK.

Ası. Papua New Guinea.

\section{Cresponea Egea \& Torrente; Roccellaceae}

Cresponea chloroconia (Tuck.) Egea \& Torrente,

Mycotaxon 48: 310 (1993).

Lecanactis deminuens (Nyl.) Vain.

ThaILAND. Trat (Koh Chang I.). Vainio (1909: 121, as Lecanactis deminuens).

SPECIMEN. TUR-V.

ASIA. -

Crocynia A. Massal.; Lecideaceae

Crocynia pyxinoides Nyl., Sert. Lich. Trop. Labuan

Singapore: 37 (1891).

Thalland. Chiang Mai (Doi Suthep N.P., Doi Inthanon N.P.), Nakhon Ratchasima (Khao Yai N.P.), Uthai Thani (Huay Kha Khaeng Wildlife Sanctuary). Wolseley \& Aguirre-Hudson (1995: 320; 1997a: 333, 339; 1997b: 356), Boonpragob et al. (1998: 217).

SPECIMENS. BM, RAMK.

AsIA. Malaysia, Singapore.

\section{Cryptothecia Stirt.; Arthoniaceae}

Cryptothecia candida (Kremp.) R. Sant., Symb. Bot. Upsal. 12(1): 65 (1952).

Thailand. Nakhon Ratchasima (Khao Yai N.P.). Boonpragob et al. (1998: 217).

SPECIMEN. RAMK.

AsIA. India, Indonesia, Malaysia, Sri Lanka.

Cryptothecia obtecta Patw. \& Sethy, Biovigyanam 13(2): 46 (1987).

THAILAND. Northern Thailand. Wolseley (1991: 240).

SPECIMEN. BM.

Ası. India.

\section{Dichosporidium Pat.; Chiodectonaceae}

Dichosporidium boschianum (Mont.) Thor, Opera Bot. 103: 64 (1990).

THAILAND. Nakhon Ratchasima (Khao Yai N.P.). Boonpragob et al. (1998: 217).

SPECIMEN. RAMK.

AsIA. China (Hong Kong), India, Malaysia, Philippines, Singapore, Sri Lanka.

Dictyonema C. Agardh ex Kunth; Meruliaceae

Dictyonema sericeum (Sw.) Berk., J. Bot. (London) 2: 639 (1843).

Dictyonema sericeum f. thelephora (Spreng.) Parm.

Thailand. Trat (Koh Chang I.). Vainio (1909: 151), Parmasto (1978: 110, 111).

SPECIMEN. TUR-V.

ASIA. China (incl. Hong Kong), Indonesia, Papua New Guinea, Philippines, Taiwan.

Dirinaria (Tuck.) Clem.; Physciaceae

Dirinaria aegialita (Ach.) B. Moore, Bryologist 71: 248 (1968).

THAILAND. Chiang Mai ( Doi Inthanon N.P., Chiang Dao N.P.), Lampang (Doi Khun Tan N.P., Doi Pae Luang N.P.). Wolseley \& Aguirre-Hudson (1997a: 333).

SPECIMENS. BM, CMU.

AsIA. China (Hong Kong), India, Indonesia, Malaysia, Philippines, Sri Lanka, Taiwan, Vietnam.

Dirinaria applanata (Fée) D.D. Awasthi, in Awasthi \& Agarwal, J. Indian Bot. Soc. 49: 135 (1970).

THAILAND. Khiri Khan, Uthai Thani (Huay Kha Khaeng Wildlife Sanctuary). Wolseley \& Aguirre-Hudson (1997a: 333).

SPECIMENS. BM.

AsıA. China (incl. Hong Kong), India, Indonesia, Malaysia, Papua New Guinea, Philippines, Sri Lanka, Taiwan, Vietnam.

Dirinaria confusa D.D. Awasthi, Biblioth. Lichenol. 2: 56 (1975).

ThaILAND. Fang (northern Thailand). Yoshimura (1978: 35).

SPECIMEN. C.

Asia. China, Papua New Guinea, Taiwan.

Dirinaria consimilis (Stirt.) D.D. Awasthi, in Awasthi \& Agarwal, J. Indian Bot. Soc. 49: 135 (1970).

ThAiland. Chiang Mai (Doi Suthep N.P., Doi Inthanon N.P., Chiang Dao N.P.), Sara Buri, Uthai Thani (Huay Kha Khaeng Wildlife Sanctuary). Awasthi (1975: 93), Wolseley \& AguirreHudson (1995: 321; 1997a: 333; 1997b: 355).

SPECIMENS. BM, CMU.

ASIA. India, Indonesia, Malaysia, Myanmar, Vietnam. 
Dirinaria picta (Sw.) Clem. \& Shear, Gen. Fung.: 323 (1931).

Physcia picta $\mathrm{Nyl}$.

ThaIlAND. Chiang Mai (Queen Sirikit Botanical Garden), Khiri Khan, Trat (Koh Chang I.). Vainio (1909: 112, as Physcia picta), Paulson (1930: 100, as P. picta), Mongkonsuk et al. (1996), Wolseley \& Aguirre-Hudson (1995: 321).

SPECIMENS. BM, TUR-V, Herbarium Queen Sirikit Botanical Garden.

ASIA. China (Hong Kong), India, Indonesia, Malaysia, Myanmar, Papua New Guinea, Philippines, Singapore, Sri Lanka, Taiwan, Vietnam.

\section{Echinoplaca Fée; Gomphillaceae}

Echinoplaca epiphylla Fée, Essai Crypt. Écorc. Offic.: 93 (1824).

THAILAND. Nakhon Ratchasima (Khao Yai N.P.). Boonpragob et al. (1998: 217).

SPECIMEN. RAMK.

AsIA. China (Hong Kong), India, Indonesia, Papua New Guinea, Philippines, Vietnam.

Echinoplaca pellicula (Müll. Arg.) R. Sant., Symb. Bot. Upsal. 12(1): 367 (1952).

THAILAND. Nakhon Ratchasima (Khao Yai N.P.). Boonpragob et al. (1998: 217).

SPECIMEN. RAMK.

ASIA. China, Indonesia, Papua New Guinea, Philippines, Sri Lanka, Vietnam.

Endocarpon Hedwig; Verrucariaceae

Endocarpon adscendens Müll. Arg., Bull. Trav. Soc. Murith. Valais 10: 58 (1881).

THAiland. Nakhon Ratchasima (Khao Yai N.P.). Boonpragob et al. (1998: 217).

SPECIMEN. CANB.

ASIA. -

Enterographa Fée; Chiodectonaceae

[Enterographa] Chiodecton pallidellum var. olivaceoalba Vain., Bot. Tidsskr. 29: 138 (1909).

Thailand. Trat (Koh Chang I.). Vainio (1909: 138), Thor (1990: 86).

SPECIMEN. TUR-V (not located).

ASIA. -

[Enterographa] Chiodecton pallidellum var. olivaceostraminea Vain., Bot. Tidsskr. 29: 137 (1909).

ThaILAND. Trat (Koh Chang I.). Vainio (1909: 137), Thor (1990: 86).

SPECIMEN. TUR-V (holotype).

ASIA. -
Eremothecella Syd; Arthoniaceae

Eremothecella macrocephala (R. Sant.) Thor, Lücking \& Matsumoto, Symb. Bot. Upsal. 32(3): 39 (2000).

Stirtonia macrocephala $\mathrm{R}$. Sant.

ThaILAND. Nakhon Ratchasima (Khao Yai N.P.). Boonpragob et al. (1998: 217, as Stirtonia macrocephala).

SPECIMENS. RAMK.

ASIA, Indonesia, Malaysia, Papua New Guinea, Philippines.

Erioderma Fée; Pannariaceae

Erioderma sorediatum D.J. Galloway \& P.M. Jørg., Lichenologist 7: 139 (1975).

ThaIland. Chiang Mai (Doi Suthep N.P.), Nakhon Nayok (Khao Yai N.P.). Wolseley \& Aguirre-Hudson (1995: 321; 1997a: 339), Boonpragob et al. (1998: 217).

SPECIMENS. BM, RAMK.

ASIA. Malaysia, Papua New Guinea, Sri Lanka.

Eschatogonia Trevis., ?Acarosporaceae

Eschatogonia prolifera (Mont.) R. Sant., in Swinscow \& Krog, Macrolichens East Africa: 86 (1988).

THAiland. Chiang Mai (Doi Inthanon N.P.), Lampang (Wiang Kosai N.P.), Nakhon Ratchasima (Khao Yai N.P.), Satun (Thaleban N.P.), Uthai Thani (Huay Kha Kaeng Wildlife Sanctuary). Boonpragob et al. (1998: 217).

SPECIMENS. BM, RAMK.

ASIA. -

Everniastrum Hale ex Sipman; Parmeliaceae

Everniastrum cirrhatum (Fr.) Hale ex Sipman, Mycotaxon 26: 237 (1986).

Parmelia cirrhata Fr.

Cetrariastrum cirrhatum (Fr.) W.L. Culb. \& C.F. Culb.

Parmelia vulgaris Vain.

Thalland. Chiang Mai (Doi Suthep, Doi Inthanon, Chiang Dao N.P.), Loei (Phu Luang). Vainio (1921: 38, as Parmelia vulgaris), Sato (1962: 3, as P. cirrhata), Moon et al. (2000a: 101, as Cetrariastrum cirrhatum).

SPECIMENS. BM, CMU, TNS, TUR-V.

ASIA. China, India, Indonesia, Nepal, Sri Lanka, Taiwan.

Everniastrum nepalense (Taylor) Hale ex Sipman, Mycotaxon 26: 239 (1986).

Cetrariastrum nepalense (Taylor) W.L. Culb. \& C.F. Culb. Parmelia americana (Meyen \& Flotow) Mont.

ThaIland. Chiang Mai (Doi Suthep N.P., Doi Inthanon N.P., Chiang Dao N.P., Maetang), Yala (Banglang N.P.). Vainio (1921:38, as Parmelia americana), Sato (1962: 6, as P. americana), Wolseley \& Aguirre-Hudson (1995: 321; 1997a: 333), Moon et al. (2000a: 101, as Cetrariastrum nepalense). 
SPECIMENS. BM, CMU, PSU, RAMK, TNS, Kurokawa, Lich. Rar. Crit. Exs. No. 78.

ASIA. China, India, Indonesia, Nepal.

Everniastrum scabridum Elix \& Pooprang, in Pooprang et al., Mycotaxon 71: 112 (1999).

THAILAND. Chiang Mai (Doi Suthep N.P.). Pooprang et al. (1999: 112).

SPECIMENS. BM, CMU, RAMK (holotype).

ASIA. -

Everniastrum sorocheilum (Vain.) Hale ex Sipman, Mycotaxon 26: 242 (1986).

Parmelia sorocheila Vain.

ThaIlAND. Chiang Mai (Doi Suthep N.P.). Sato (1962: 4, as Parmelia sorocheila Vain.).

SPECIMEN. OSA.

AsIA. China, Indonesia, Philippines, Taiwan.

Everniastrum vexans (Zahlbr. ex W.L. Culb. \& C.F. Culb.) Hale ex Sipman, Mycotaxon 26: 242 (1986).

Cetrariastrum vexans Zahlbr. ex W.L. Culb. \& C.F. Culb.

ThaIlAND. Chiang Mai (Doi Suthep N.P., Doi Inthanon N.P.), Loei (Phu Luang N.P.). Wolseley \& Aguirre-Hudson (1995: 318; 1997a: 333), Moon et al. (2000a: 101, as Cetrariastrum vexans).

SPECIMENS. BM, TNS.

AsıA. China, India, Indonesia, Papua New Guinea, Philippines, Taiwan.

Fuscopannaria P.M. Jørg.; Pannariaceae

Fuscopannaria siamensis P.M. Jørg. \& Wolseley, in Jørgensen, J. Hattori Bot. Lab. 89: 256 (2000).

THAILAND. Chiang Mai (Doi Inthanon N.P.). Jørgensen (2000: 256).

SPECIMENS. BM (holotype).

ASIA. -

Glyphis Ach.; Graphidaceae

Glyphis cicatricosa var. confluens (Zenker) Zahlbr., Denkschr. Kaiserl. Akad. Wiss., Wien Math.-Naturwiss. Cl. 83: 112 (1909).

Graphis cicatricosa var. confluens (Zenker) Vain.

ThaIland. Trat (Koh Chang I.), Uthai Thani (Huay Kha Khaeng Wildlife Sanctuary). Vainio (1909: 136, as Graphis cicatricosa var. confluens).

SPECIMENS. BM, TUR-V.

ASIA. Vietnam.

Graphidastra (Redinger) Thor; Chiodectonaceae

Graphidastra byssiseda (Müll. Arg.) Thor, Opera Bot. 103: 80 (1990).
Thailand. Chiang Mai (Doi Inthanon N.P., Doi Suthep N.P.). Wolseley \& Aguirre-Hudson (1997: 333).

SPECIMENS. BM.

AsIA. India, Indonesia, Nepal.

Graphina Müll. Arg.; Graphidaceae

Graphina boschiana Müll. Arg., Flora 65: 385 (1882).

Graphis concolor Nyl.

Thailand. Trat (Koh Chang I.). Vainio (1909: 128, as Graphis concolor).

SPECIMENS. TUR-V.

AsıA. India, Indonesia, Sri Lanka.

Graphina columbiana (Nyl.) Zahlbr., Cat. Lich. Univ. 2: 402 (1924).

Graphis obtecta var. columbiana Nyl.

Thalland. Chiang Mai (Doi Suthep N.P.). Vainio (1921: 51, as Graphis obtecta var. columbiana).

SPECIMEN. TUR-V.

ASIA. India.

Graphina consimilis (Vain.) Zahlbr., Cat. Lich. Univ. 2: 402 (1923).

Graphis consimilis Vain.

ThaIlAnd. Trat (Koh Chang I.). Vainio (1907: 177; 1909: 127, both as Graphis consimilis).

SPECIMEN. TUR-V (holotype).

AsIA. Philippines.

Graphina fissurinoidea (Vain.) Zahlbr., Cat. Lich. Univ. 2: 406 (1923).

Graphis fissurinoidea (Nyl.)Vain.

ThaILAND. Trat (Koh Chang I.). Vainio (1909: 128, as Graphis fissurinoidea).

SPECIMEN. TUR-V.

ASIA. -

Graphina glaucorufa (Vain.) Zahlbr., Cat. Lich. Univ. 2: 407 (1923).

Graphis glaucorufa Vain.

ThAILAND. Chiang Mai (Doi Suthep N.P.). Vainio (1921: 51, as Graphis glaucorufa).

SPECIMEN. TUR-V (holotype).

ASIA. -

Graphina intermedians (Vain.) Zahlbr., Cat. Lich. Univ. 2: 411 (1923).

Graphis intermedians Vain.

ThaILAND. Chiang Mai (Doi Suthep N.P.). Vainio (1921: 50, as Graphis intermedians). 
SPECIMEN. TUR-V (holotype).

ASIA. -

Graphina intricata (Eschw.) Müll. Arg., Flora 71: 510 (1888).

Graphis subintricata Kremp.

Graphis extricata Vain.

ThaIlAND. Trat (Koh Chang I.). Vainio (1909: 126, as Graphis subintricata and G. extricata).

SPECIMENS. TUR-V.

ASIA. Sri Lanka.

Graphina ruiziana var. gracilior Müll. Arg., Flora 65: 385 (1882).

Graphis ruiziana var. gracilior Vain.

ThaIland. Trat (Koh Chang I.). Vainio (1909: 125, as Graphis ruiziana var. gracilior).

SPECIMENS. TUR-V.

ASIA. Indonesia.

Graphina simplex (Vain.) Zahlbr., Cat. Lich. Univ. 2: 425 (1923).

Graphis simplex Vain.

Thailand. Trat (Koh Chang I.). Vainio (1907: 127 as Graphis simplex)

SPECIMEN. TUR-V (holotype).

ASIA. -

Graphina streblocarpa var. pauperior Vain., Suom. Elainja Kasvit. Seuran Van. Julk. 1(3): 50 (1921).

ThaIland. Chiang Mai (Doi Suthep N.P.). Vainio (1921: 50).

SPECIMEN. TUR-V (holotype).

ASIA. -

Graphina subobtecta var. feracior (Vain.) Zahlbr., Cat. Lich. Univ. 2: 427 (1923).

Graphis subobtecta var. feracior Vain.

ThaILAND. Chiang Mai (Doi Suthep N.P.). Vainio (1921: 51, as Graphis subobtecta var. feracior).

SPECIMEN. TUR-V (holotype).

ASIA. -

Graphis Adans.; Graphidaceae

Graphis afzelii Ach., Syn. Meth. Lich.: 85 (1814).

ThAILAND. Chiang Mai (Baw Luang), Nakhon Ratchasima (Khao Yai N.P.), Uthai Thani (Huay Kha Khaeng Wildlife Sanctuary). Boonpragob et al. (1998: 217).

SPECIMENS. RAMK, BM.

AsIA. China (incl. Hong Kong), India, Indonesia, Malaysia, Singapore, Sri Lanka, Vietnam.
Graphis albonitens Müll. Arg., Hedwigia 30: 53 (1891)

Thailand. Chumphon (Koh Tao I.). Paulson (1930: 99).

SPECIMEN. BM.

ASIA. -

Graphis glaucocinerea Vain., Hedwigia 46: 178 (1907).

Thailand. Trat (Koh Chang I.). Vainio (1907: 178; 1909: 135).

SPECIMENS. TUR-V, FH (both syntypes).

ASIA. -

Graphis hossei Vain., Suom. Elain-ja Kasvit. Seuran Van. Julk. 1(3): 54 (1921).

Thailand. Chiang Mai (Doi Suthep N.P.). Vainio (1921: 54), Patwardhan \& Kulkarni (1976: 127).

SPECIMEN. TUR-V (holotype).

ASIA. India.

Graphis leptospora Vain., Suom. Elain-ja Kasvit. Seuran Van. Julk. 1(3): 53 (1921).

Thalland. Chiang Mai (Doi Suthep N.P.). Vainio (1921: 53).

SPECIMENS. TUR-V (holotype).

ASIA. -

Graphis lineola Ach., Lichenogr. Universalis: 264 (1810).

ThaIland. Trat (Koh Chang I.). Vainio (1909: 134).

SPECIMEN. TUR-V.

AsIA. China (incl. Hong Kong), India, Indonesia, Papua New Guinea, Philippines.

Graphis ochrocheila Vain., Hedwigia 46: 178 (1907).

Thailand. Trat (Koh Chang I.). Vainio (1907: 178; 1909: 135)

SPECIMEN. TUR-V (holotype).

ASIA. -

Graphis pyrrhocheiloides Zahlbr., Cat. Lich. Univ. 2: 321 (1923).

Graphis pyrrhocheila Vain.

ThaIland. Trat (Lem Ngob). Vainio (1907: 179; 1909: 136, both as Graphis pyrrhocheila).

SPECIMEN. TUR-V (holotype).

ASIA. India, Indonesia.

Graphis sauroidea Leight., Trans. Linn. Soc. London 25: 452 (1866) .

Thailand. Trat (Koh Chang I.). Vainio (1909: 133).

SPECIMENS. TUR-V.

ASIA. -

Graphis siamensis Vain., Suom. Elain-ja Kasvit. Seuran Van. Julk. 1(3): 52 (1921). 
ThaIland. Chiang Mai (Doi Suthep). Vainio (1921: 52).

SPECIMENS. TUR-V (holotype).

ASIA. -

Graphis striatula (Ach.) Spreng., Syst. Veg., 16th ed, 4: 250 (1827).

Thailand. Trat (Koh Chang I.). Vainio (1909: 134).

SPECIMENS. TUR-V.

ASıA. China, India, Indonesia, Malaysia, Sri Lanka.

Graphis subdisserpens Nyl., Bull. Soc. Linn. Normandie, sér. 2, 7: 16 (1874).

Thailand. Trat (Koh Chang I.). Vainio (1909: 132).

SPECIMENS. TUR-V.

ASIA. India.

Graphis subdisserpens f. arbusculaeformis Vain., Bot. Tidsskr. 29: 132 (1909).

Thailand. Trat (Koh Chang I.). Vainio (1909: 132).

SPECIMENS. TUR-V (holotype).

ASIA. - -

Graphis tenella Ach., Syn. Meth. Lich.: 81 (1814).

Thalland. Chiang Mai (Doi Suthep N.P.). Vainio (1909: 133; 1921: 54).

SPECIMENS. TUR-V.

ASIA. China, India, Indonesia, Sri Lanka.

Graphis tenella var. epiphaea Vain., Bot. Tidsskr. 29: 133 (1909).

ThaILAND. Trat (Koh Chang I.). Vainio (1909: 133).

SPECIMENS. TUR-V (holotype).

ASIA. -

Graphis tenella var. leptocarpoides Vain., Bot. Tidsskr. 29: 133 (1909).

Thailand. Trat (Koh Chang I.). Vainio (1909: 133).

SPECIMENS. TUR-V (syntypes).

ASIA. -

Graphis tenuis Vain., Suom. Elain-ja Kasvit. Seuran Van. Julk. 1(3): 53 (1921).

Thailand. Chiang Mai (Doi Suthep N.P.). Vainio (1921: 53).

SPECIMENS. TUR-V (syntypes).

ASIA. -

Graphis trichospora Vain., Suom. Elain-ja Kasvit. Seuran Van. Julk. 1(3): 52 (1921).

ThaILAND. Chiang Mai (Doi Suthep N.P.). Vainio (1921: 52).

SPECIMEN. TUR-V (holotype).

ASIA. -
Gyalideopsis Veězda; Gomphillaceae

Gyalideopsis rubescens Veĕzda, Folia Geobot. Phytotax. 14: 67 (1979).

ThaIlAND. Chiang Mai (Doi Inthanon N.P.), Nakhon Ratchasima (Khao Yai N.P.). Wolseley \& Aguirre-Hudson (1997a: 333), Boonpragob et al. (1998: 217).

SPECIMENS. BM, RAMK.

ASIA. -

Gymnoderma Nyl.; Cladoniaceae

Gymnoderma coccocarpum Nyl., Syn. Meth. Lich. 2: 26 (1863).

THAILAND. Nakhon Nayok. Yoshimura \& Sharp (1968: 635640), Hawksworth \& Yoshimura (1973: 503), Yoshimura (1982: 84), Kashiwadani \& Gradstein (1982: 80).

SPECIMENS. US.

ASIA. China, India, Indonesia, Malaysia, Taiwan.

Gyrostomum Fr.; Thelotremataceae

Gyrostomum scyphuliferum (Ach.) Nyl., Ann. Sci. Nat. Bot., sér. 4, 16: 96 (1862).

THAILAND. Chiang Mai (Doi Inthanon N.P.), Nakhon Ratchasima (Khao Yai N.P.), Trat (Koh Chang I.). Vainio (1909: 121), Wolseley \& Aguirre-Hudson (1997a: 333), Boonpragob et al. (1998: 217).

SPECIMENS. BM, RAMK, TUR-V.

ASIA. China (incl. Hong Kong), India, Indonesia, Malaysia, Philippines, Taiwan, Vietnam.

Haematomma A. Massal; Haematommataceae

Haematomma collatum (Stirt.) C.W. Dodge, Beih. Nova Hedwigia 38: 41 (1971).

ThaIland. Chiang Mai (Doi Suthep N.P., Doi Inthanon N.P.), Lampang (Doi Khun Tan N.P.), Uthai Thani (Khao Nang Ram Wildlife Sanctuary). Staiger \& Kalb (1995: 89).

SPECIMENS. BKF, BM, CMU, COLO.

ASIA. India, Indonesia, Papua New Guinea, Sri Lanka.

Haematomma puniceum (Ach.) A. Massal., Atti Reale Ist. Veneto, Sci. Lett. Arti, ser. 3, 5: 253 (1860).

Thailand. Chiang Mai (Doi Suthep N.P., Doi Inthanon N.P.), Uthai Thani (Khao Nang Ram Wildlife Sanctuary). Vainio (1921: 43), Wolseley \& Aguirre-Hudson (1997a: 339).

SPECIMENS. BKF, BM, CMU, TUR-V.

ASIA. China, India, Indonesia, Singapore, Sri Lanka, Taiwan, Vietnam.

Haematomma wattii (Stirt.) Zahlbr., Cat. Lich. Univ. 5: 776 (1928).

Thailand. Chiang Mai (Doi Suthep N.P., Chiang Dao N.P.). Staiger \& Kalb (1995: 89), Wolseley \& Aguirre-Hudson (1997a: $333,339)$ 
SPECIMENS. BM, CMU.

AsIA. India, Indonesia, Malaysia, Papua New Guinea, Philippines.

Hafellia Kalb, H. Mayrhofer \& Scheidegger;

Physciaceae

Hafellia disciformis (Fr.) Marbach \& H. Mayrhofer, in Marbach, Biblioth. Lichenol. 74: 259 (2000).

Buellia disciformis (Fr.) Mudd

ThaIlAND. Chiang Mai (Doi Suthep N.P.). Vainio (1921: 45, as Buellia disciformis).

SPECIMENS. TUR-V.

AsIA. China, India, Indonesia, Vietnam.

Heterodermia Trevis.; Physciaceae

Heterodermia albicans (Pers.) Swinscow \& Krog, Lichenologist 8: 113 (1976).

Physcia crispa var. mollescens (Nyl.) Vain.

THAILAND. Chiang Mai (Queen Sirikit Botanical Garden), Trat (Koh Chang I.). Vainio (1909: 111, as Physcia crispa var. mollescens), Mongkonsuk et al. (1996: 17).

SPECIMENS. TUR-V, Queen Sirikit Botanical Garden Herbarium. ASIA. China, Malaysia, Philippines.

Heterodermia dendritica (Pers.) Poelt, Nova Hedwigia 9: 31 (1965).

Anaptychia dendritica Pers.

THAILAND. Hase Plantation, probably Laos. Kurokawa (1962: 54; 1973: 602, both as Anaptychia dendritica).

SPECIMEN. TNS.

AsIA. China, India, Indonesia, Papua New Guinea, Philippines, Taiwan.

Heterodermia diademata (Taylor) D.D. Awasthi, Geophytology 3: 113 (1973).

ThaILAND. Chiang Mai (Doi Inthanon N.P., Doi Suthep N.P., Chiang Dao N.P.). Wolseley \& Aguirre-Hudson (1997b: 356).

SPECIMENS. BM.

AsIA. India, China, Malaysia, Papua New Guinea, Sri Lanka.

Heterodermia esorediata (Vain.) Du Rietz \& Lynge, in Lynge, Videnskaps. I. Mat.-Naturv. Kl. 16: 14 (1924).

Anaptychia esorediata Vain.

THAIlAnd. Locality not given. Kurokawa (1959: 179, as Anaptychia esorediata).

SPECIMEN. TNS (not seen).

AsIA. India, Indonesia, Philippines.

Heterodermia fragilissima (Kurok.) J.-C. Wei \& Y.-M. Jiang, Lich. Xizang: 111 (1986).
THAILAND. Chiang Mai (Doi Inthanon N.P.). Wolseley \& AguirreHudson (1997a: 333).

SPECIMENS. BM.

Ası. China.

Heterodermia hypocaesia (Yasuda) D.D. Awasthi, Geophytology 3: 113 (1973).

Anaptychia hypocaesia Yasuda

THAILAND. Chiang Mai (Doi Suthep N.P., Maetang District), Loei (Phu Kradung N.P.). Kurokawa (1973: 603, as Anaptychia hypocaesia).

SPECIMENS. TNS.

AsIA. China, Indonesia, Papua New Guinea, Philippines, Taiwan.

Heterodermia hypoleuca (Ach.) Trevis., Atti Soc. Ital. Sci. Nat. 11: 615 (1868).

Anaptychia hypoleuca (Ach.) A. Massal.

ThAILAND. Chiang Mai (Doi Suthep N.P., Doi Inthanon N.P., Queen Sirikit Botanical Garden), Uthai Thani (Huay Kha Khaeng Wildlife Sanctuary). Vainio (1921: 45, as Anaptychia hypoleuca), Mongkonsuk et al. (1996: 17).

SPECIMENS. BM, TUR-V, Queen Sirikit Botanical Garden Herbarium.

ASIA. China, India, Malaysia, Papua New Guinea, Sri Lanka, Taiwan.

Heterodermia incana (Stirt.) D.D. Awasthi, Geophytology 3: 114 (1973).

Anaptychia incana (Stirt.) Zahlbr.

THAILAND. Hase Plantation, probably Laos. Kurokawa (1962: 92, as Anaptychia incana).

SPECIMEN. TNS.

AsIA. China, India, Indonesia, Taiwan.

Heterodermia leucomelos (L.) Poelt, Nova Hedwigia 9: 31 (1965).

Anaptychia leucomelaena (L.) A. Massal.

Anaptychia neoleucomelaena Kurok.

ThaIlAND. Chiang Mai (Doi Suthep N.P., Doi Inthanon N.P.), Trat (Koh Chang I., Lem Dan). Vainio (1921: 44, as Anaptychia leucomelaena), Kurokawa (1961: 51, as A. neoleucomelaena), Wolseley \& Aguirre-Hudson (1997a: 333).

SPECIMENS. BM, TUR-V.

AsIA. China, India, Indonesia, Malaysia, Myanmar, Papua New Guinea, Philippines, Sri Lanka, Taiwan.

Heterodermia microphylla (Kurok.) Skorepa, Bryologist 75: 490 (1972).

THAILAND. Chiang Mai (Doi Inthanon N.P.), Nakhon Nayok (Khao Yai N.P.). Boonpragob et al. (1998: 217).

SPECIMENS. BM, RAMK.

ASIA. China, India, Papua New Guinea. 
Heterodermia pandurata (Kurok.) J.-C. Wei, Enum. Lich. China: 112 (1991).

Anaptychia pandurata Kurok.

THAILAND. Hase Plantation, probably Laos. Kurokawa (1962: 95-96).

SPECIMENS. TNS.

ASIA. Taiwan.

Heterodermia speciosa (Wulfen) Trevis., Atti Soc. Ital. Sci. Nat. 11: 614 (1868).

Anaptychia speciosa (Wulfen) A. Massal.

ThaIlAND. Chiang Mai (Doi Suthep N.P., Doi Inthanon N.P.). Vainio (1921: 45, as Anaptychia speciosa).

SPECIMENS. BM, TUR-V.

AsIA. China (incl. Hong Kong), India, Indonesia, Malaysia, Sri Lanka.

Hypogymnia (Nyl.) Nyl.; Hypogymniaceae

[Hypogymnia] Parmelia coilocarpa Vain., Suom. Elain-ja Kasvit. Seuran Van. Julk. 1(3): 40 (1921), auct. non Stirt.

ThaILAND. Chiang Mai (Doi Suthep). Vainio (1921: 40, as Parmelia coilocarpa).

SPECIMENS. BM, TUR-V (syntype).

ASIA. -

Hypogymnia pseudobitteriana (D.D. Awasthi) D.D. Awasthi, in Awasthi \& Singh, Geophytology 1: 101 (1972).

ThaILAND. Chiang Mai (Doi Suthep Pui N.P., Doi Inthanon N.P.). Wolseley \& Aguirre-Hudson (1995: 318).

SPECIMENS. BM.

ASIA. India, Malaysia, Papua New Guinea, Taiwan.

Hypogymnia subphysodes (Kremp.) Filson, Victorian Nat. 87: 327 (1970)

Parmelia subphysodes Kremp.

THAILAND. Chiang Mai (Doi Suthep, N.P.). Vainio (1921: 40, as Parmelia subphysodes), Wolseley \& Aguirre-Hudson (1997a: 333).

SPECIMENS. BM, TUR-V.

ASIA. -

Hypogymnia vittata (Ach.) Gas., Acta Soc. Linn. Bordeaux 53: 66 (1898).

ThaILAND. Chiang Mai (Doi Inthanon N.P.). Wolseley \& AguirreHudson (1997a: 333, 337).

SPECIMENS. BM.

ASIA. China, India, Malaysia, Nepal, Taiwan.

Hypotrachyna (Vain.) Hale; Parmeliaceae

Hypotrachyna adducta (Nyl.) Hale, Phytologia 28: 341

(1974).
ThaIland. Chiang Mai (Doi Inthanon N.P.). Pooprang et al. (1999: 119), Moon et al. (2000a: 101).

SPECIMENS. RAMK, TNS.

AsIA. China, India, Malaysia, Nepal, Philippines, Taiwan.

Hypotrachyna adjuncta (Hale) Hale, Phytologia 28: 341 (1974).

Parmelia exsecta Taylor.

Hypotrachyna exsecta (Taylor) Hale.

Thailand. Chiang Mai (Doi Suthep N.P., Doi Inthanon N.P.), Loei (Phu Luang N.P), Phitsanulok. Sato (1962: 3, as Parmelia exsecta) Kurokawa (1986: 265, as Hypotrachyna exsecta), Pooprang et al. (1999: 120), Moon et al. (2000a: 101, and as H. exsecta).

SPECIMENS. BKF, BM, CMU, RAMK, TNS.

ASIA. India, Malaysia, Nepal, Philippines, Sri Lanka, Taiwan.

Hypotrachyna bahiana (Nyl.) Hale, Smithsonian Contr. Bot. 25: 23 (1975).

Parmelia bahiana Nyl.

Thailand. Chiang Mai (Doi Suthep). Vainio (1921: 38, as Parmelia bahiana).

SPECIMEN. TUR-V.

ASIA. -

Hypotrachyna chlorobarbatica Elix \& Pooprang, in Pooprang, Boonpragob \& Elix, Mycotaxon 72: 113 (1999).

THAILAND. Nakhon Ratchasima (Khao Yai N.P.). Pooprang et al. (1999: 113).

SPECIMENS. CANB (isotype), RAMK (holotype).

ASIA. -

Hypotrachyna coorgiana Patw. \& Prabhu, Bryologist 80: 348 (1997).

ThaIland. Nakhon Ratchasima (Khao Yai N.P.), Phitsanulok (Phu Hin Rongkla N.P.). Pooprang et al. (1999: 120).

SPECIMENS. RAMK.

AsIA. India.

Hypotrachyna crenata (Kurok.) Hale, Phytologia 28: 341 (1974).

Parmelia crenata Kurok.

ThAILAND. Chiang Mai (Maetang District). Kurokawa (1988: 167-168, as Parmelia crenata), Moon et al. (2000a: 101).

SPECIMENS. TNS.

ASIA. India, Indonesia, Taiwan.

Hypotrachyna dactylifera (Vain.) Hale, Smithsonian Contr. Bot. 25: 30 (1975).

ThAILAND. Chiang Mai (Doi Inthanon N.P., Doi Suthep N.P.). Moon et al. (2000a: 101).

SPECIMENS. TNS.

ASIA. - 
Hypotrachyna ducalis (Jatta) Hale, Smithsonian Contr. Bot. 25: 33 (1975).

THAILAND. Chiang Mai (Doi Suthep N.P.), Nakhon Ratchasima (Khao Yai N.P.). Wolseley \& Aguirre-Hudson (1997a: 335), Boonpragob et al. (1998: 217).

SPECIMENS. BKF, BM, CMU, RAMK.

ASIA. -

Hypotrachyna granulans K.H. Moon, Kurok. \& Kashiw., Bull. Natn. Sci. Mus., Tokyo, ser. B, 26: 135 (2000).

THAILAND. Chiang Mai (Doi Inthanon N.P.). Moon et al. (2000b: 135-137).

SPECIMENS. TNS (holotype), RAMK (isotype).

ASIA.

Hypotrachyna imbricatula (Zahlbr.) Hale, Smithsonian Contr. Bot. 25: 41 (1975).

Parmelia imbricatula Zahlbr.

Thailand. Chiang Mai (Doi Suthep N.P., Doi Pacho, Maetang), Loei (Ban Sui, Phu Kradung). Hale (1971: 16, as Parmelia imbricatula), Moon et al. (2000a: 101).

SPECIMENS. TNS.

AsIA. India, Malaysia, Philippines, Taiwan.

Hypotrachyna immaculata (Kurok.) Hale, Smithsonian Contr. Bot. 25: 41 (1975).

Thailand. Phitsanulok (Phu Hin Rongkla N.P.). Pooprang et al. (1999: 120).

SPECIMEN. RAMK.

ASIA. India.

Hypotrachyna infirma (Kurok.) Hale, Phytologia 28: 341 (1974).

Parmelia infirma Kurok.

Thailand. Loei (Ban Sui, Phu Kradung). Hale \& Kurokawa (1964: 179, as Parmelia infirma), Awasthi (1977: 177), Moon et al. (2000a: 101).

SPECIMEN. TNS.

AsIA. India, Nepal, Taiwan.

Hypotrachyna kingii (Hale) Hale, J. Jap. Bot. 43: 324 (1968).

Parmelia kingii Hale.

ThAILAND. Nakhon Nayok (Khao Yai N.P.). Hale (1968: 324, as Parmelia kingii), Moon et al. (2000a: 101).

SPECIMENS. TNS (isotype), US (holotype).

AsIA. Indonesia.

Hypotrachyna massartii (Hue) Hale, Phytologia 28: 341 (1974).

Thailand. Loei (Phu Kradung). Moon et al. (2000a: 102).
SPECIMENS. TNS.

ASIA. -

Hypotrachyna masonhalei Patw. \& Prabhu, Bryologist 80: 348 (1997).

ThaILAnd. Chiang Mai (Doi Suthep N.P.). Pooprang et al. (1999: 121).

SPECIMEN. RAMK.

ASIA. India.

Hypotrachyna orientalis (Hale) Hale, Phytologia 28: 341 (1974).

Parmelia orientalis Hale.

THAILAND. Chiang Mai (Doi Suthep N.P.), Nakhon Nayok (Khao Yai N.P.). Hale (1971: 435, as Parmelia orientalis Hale), Culberson \& Hale (1973: 166, as P. orientalis), Wolseley \& Aguirre-Hudson (1995: 318), Boonpragob et al. (1998: 217), Moon et al. (2000a: 102).

SPECIMENS. BM, CMU, KF, TNS (isotype), UPS (isotype), US (holotype).

ASIA. India, Malaysia, Papua New Guinea, Philippines.

Hypotrachyna osseoalba (Vain.) Y.S. Park \& Hale, Taxon 38: 88 (1989).

Parmelia osseoalba Vain.

Hypotrachyna formosana auct. non (Zahlbr.) Hale.

THAIland. Chiang Mai (Doi Suthep N.P., Doi Inthanon N.P., Maetang), Loei (Ban Sui, Phu Kradung N.P.), Nakhon Ratchasima (Khao Yai N.P.). Vainio (1921:39, as Parmelia osseoalba), Wolseley \& Aguirre-Hudson (1995: 318, as Hypotrachyna formosana), Boonpragob et al. (1998: 217, as H. formosana), Moon et al. (2000a: 102).

SPECIMENS. BKF, BM, CMU, RAMK, TNS, TUR-V (holotype). ASIA. China, India, Indonesia, Malaysia, Philippines, Taiwan.

Hypotrachyna physcioides (Nyl.) Hale, Smithsonian Contr. Bot. 25: 54 (1975).

THAIl ANd. Chiang Mai (Doi Suthep N.P., Doi Pacho, Maetang), Loei (Nahaew N.P., Phu Kradung). Pooprang et al. (1999: 120), Moon et al. (2000a: 102).

SPECIMENS. RAMK, TNS.

ASIA. India, Malaysia, Papua New Guinea.

Hypotrachyna ramkhamhaengiana Elix \& Pooprang, in Pooprang, Boonpragob \& Elix, Mycotaxon 72: 113 (1999).

THAILAND. Sakon Nakhon (Phu Pan N.P.). Pooprang et al. (1999: 113).

SPECIMENS. RAMK (holotype).

ASIA. -

Hypotrachyna scytodes (Kurok.) Hale, Phytologia 28: 341 (1974).

Parmelia scytodes Kurok. 
ThaIlAnd. Chiang Mai (Maetang District). Culberson \& Hale (1973: 166, as Parmelia scytodes), Moon et al. (2000a: 102).

SPECIMENS. TNS.

AsIA. India, Malaysia.

Imshaugia S.L.F. Mey.; Parmeliaceae

Inshaugia placorodia (Ach.) S.L.F. Mey., Mycotaxon 35: 189 (1989).

Parmeliopsis placorodia (Ach.) Nyl.

ThaILAND. Chiang Mai (Queen Sirikit Botanical Garden). Mongkonsuk et al. (1996: 17, as Parmeliopsis placorodia).

SPECIMENS. Queen Sirikit Botanical Garden Herbarium.

AsiA. -

Ionaspis Th. Fr.; Hymeneliaceae

Ionaspis tropica Aptroot, in Aptroot et al., Biblioth. Lichenol. 64: 73 (1997).

THAILAND. Nakhon Ratchasima (Khao Yai N.P.). Boonpragob et al. (1998: 217).

SPECIMENS. RAMK.

AsiA. Papua New Guinea.

Lasioloma R. Sant.; Ectolechiaceae

Lasioloma arachnoideum (Kremp.) R. Sant., Symb. Bot. Upsal. 12(1): 547 (1952).

THAILAND. Nakhon Ratchasima (Khao Yai N.P.). Boonpragob et al. (1998: 217).

SPECIMENS. RAMK.

AsIA. India, Indonesia, Malaysia, Papua New Guinea, Philippines, Vietnam.

Laurera Rchb.; Trypetheliaceae

Laurera benguelensis (Müll. Arg.) Zahlbr., Cat. Lich. Univ. 1: 503 (1992).

Thailand. Chiang Mai (Queen Sirikit Botanical Garden), Nakhon Nayok (Khao Yai N.P.), Uthai Thani (Huay Kha Khaeng Wildlife Sanctuary). Mongkonsuk et al. (1996: 17), Wolseley \& AguirreHudson (1997a: 339, 1997b: 356), Boonpragob et al. (1998: 217).

SPECIMENS. BM, RAMK, Queen Sirikit Botanical Garden Herbarium.

AsIA. India.

Laurera keralensis D.K. Upreti \& A. Singh, Bull. Jard. Bot. Belg. 57: 374 (1987).

Thailand. Nakhon Ratchasima (Khao Yai N.P.), Sakon Nakhon (Ratchapat Sakon). Vongshewarat et al. (1999: 227).

SPECIMENS. RAMK

ASIA. India.

Laurera megasperma (Mont.) Riddle, Bull. Torrey Bot. Club 44: 323 (1917).
Thailand. Nakhon Ratchasima (Khao Yai N.P.). Vongshewarat et al. (1999: 228).

SPECIMENS. RAMK.

AsiA. India, Papua New Guinea, Sri Lanka.

Laurera meristospora (Mont. \& Bosch) Zahlbr., Cat. Lich. Univ. 1: 505 (1922).

Thelenella interrupta Vain.

Clathroporina interrupta (Vain.) Zahlbr.

ThaIlAND. Trat (Koh Chang I.), Nakhon Ratchasima (Khao Yai N.P.). Vainio (1907: 180, 1909: 149, both as Thelenella interrupta), McCarthy (1995: 347, as Laurera aff. meristospora), Boonpragob et al. (1998: 217).

SPECIMENS. RAMK, TUR-V (holotype of $T$. interrupta).

AsiA. Indonesia, Papua New Guinea.

Laurera meristosporoides P.M. McCarthy \& Vongshewarat, in Vongshewarat et al., Mycotaxon 71: 228 (1999).

THAILAND. Phitsanulok (Phu Hin Rongkla N.P.). Vongshewarat et al. (1999: 228).

SPECIMENS. CANB (holotype).

AsIA. -

Laurera phaeomelodes (Müll. Arg.) Zahlbr., in Engler \& Prantl, Nat. Pflanzenfam. 1, 1: 71 (1903).

ThaIland. Phitsanulok (Phu Hin Rongkla N.P.). Vongshewarat et al. (1999: 230).

SPECIMENS. RAMK.

AsIA. India.

Laurera subdiscreta (Nyl.) Zahlbr., Cat. Lich. Univ. 1: 506 (1922).

Thailand. Buri Ram (Khao Kadong), Sakon Nakhon (Phu Pan N.P.). Vongshewarat et al. (1999: 230).

SPECIMENS. RAMK.

ASIA. India.

Lecanactis Körb.; Roccellaceae

Lecanactis flavisedella (Nyl.) Tehler, Willdenowia 22: 206 (1992).

ThAILAND. Chiang Mai (Doi Suthep N.P.), Uthai Thani (Huay Kha Khaeng Wildlife Sanctuary). Wolseley \& Aguirre-Hudson (1997a: 333, 339).

SPECIMENS. BM.

AsIA. India.

\section{Lecanora Ach.; Lecanoraceae}

Lecanora achroella Nyl., J. Bot. 14: 263 (1876).

Lecanora monodorae Vain.

ThaILAND. Trat (Koh Chang I.). Vainio (1909: 109, as Lecanora monodorae). 
SPECIMENS. TUR-V.

ASIA. -

Lecanora cinereocarnea (Eschw.) Vain., Étud. Class. Lich. Brésil 1: 80 (1891).

Thailand. Chiang Mai (Doi Suthep N.P.), Trat (Koh Chang I., Lem Dan). Vainio (1909: 108; 1921: 41).

SPECIMENS. TUR-V.

ASIA. Philippines, Vietnam.

Lecanora fumigata Vain., Suom. Elain-ja Kasvit. Seuran Van. Julk. 1(3): 42 (1921).

Thalland. Chiang Mai (Doi Suthep N.P.). Vainio (1921: 42).

SPECIMEN. TUR-V (holotype).

ASIA. -

Lecanora lividocarnea Vain., Philipp. J. Sci., sect. C, 8: 100 (1913).

Thailand. Chiang Mai (Doi Suthep N.P.). Vainio (1921: 41).

SPECIMENS. TUR-V.

AsIA. Philippines.

Lecanora lividoglauca Vain., Suom. Elain-ja Kasvit.

Seuran Van. Julk. 1(3): 41 (1921).

ThaIland. Chiang Mai (Doi Suthep N.P.). Vainio (1921: 41).

SPECIMEN. TUR-V (holotype).

ASIA. -

Lecanora phaeocardia Vain., Suom. Elain-ja Kasvit.

Seuran Van. Julk. 1(3): 41 (1921).

Lecanora phaeocardia var. lividofuscescens Vain.

Lecanora phaeocardia var. subcrenulans Vain.

ThaIlAnd. Chiang Mai (Doi Suthep N.P.), Nong Boa, Uthai Thani (Huay Kha Khaeng Wildlife Sanctuary). Vainio (1921: 41, 42), Lumbsch et al. (1996: 283-285).

SPECIMEN. TUR-V (holotype of Lecanora phaeocardia var. lividofuscescens)

ASIA. India, Indonesia.

Lecanora pulicaris (Pers.) Ach., Syn. Meth. Lich.: 336 (1814).

Lecanora subfusca var. chlarona Ach.

ThAILAND. Trat (Koh Chang I.). Vainio (1909: 108, as Lecanora subfusca var. chlarona).

SPECIMENS. TUR-V.

AsıA. China, India, Papua New Guinea, Philippines.

Lecanora subfusca Ach., Lichenogr. Universalis: 393 (1810).

ThaILAND. Chiang Mai (Doi Suthep N.P.), Chumphon (Koh Tao I.), Uthai Thani (Huay Kha Khaeng Wildlife Sanctuary). Paulson (1930: 99), Wolseley \& Aguirre-Hudson (1997b: 356).
SPECIMENS. BM.

AsıA. China, Sri Lanka.

Lecanora subgranulata Nyl., Lich. Nov.-Zel.: 62 (1888).

Thailand. Trat (Koh Chang I.). Vainio (1909: 108).

SPECIMENS. TUR-V.

ASIA. -

Lecidea Ach.; Lecideaceae

Lecidea inolescens Vain., Suom. Elain-ja Kasvit. Seuran Van. Julk. 1(3): 48 (1921).

Thailand. Chiang Mai (Doi Suthep N.P.). Vainio (1921: 48). SPECIMENS. TUR-V (type not located).

ASIA. -

Lecidopyrenopsis Vain.; Lichinaceae

Lecidopyrenopsis corticola Vain., Hedwigia 46: 172 (1907).

ThaILAND. Trat (Koh Chang I.). Vainio (1909: 117), Zahlbruckner (1926: 172), Henssen (1979: 485; 1989: 102).

SPECIMENS. C (isolectotype), TUR-V (lectotype).

AsIA. -

Leprocaulon Nyl.; Fungi Imperfecti

Leprocaulon arbuscula (Nyl.) Nyl., Lich. Ins. Guineensis: 8 (1889).

THAILAND. Chiang Mai (Doi Inthanon N.P., Doi Suthep N.P.), Nakhon Ratchasima (Khao Yai N.P.). Wolseley \& Aguirre-Hudson (1997a: 333), Boonpragob et al. (1998: 217).

SPECIMENS. BM, RAMK.

ASIA. China, India, Taiwan.

Leptogium (Ach.) S.F. Gray; Collemataceae

Leptogium asiaticum P.M. Jørg., Herzogia 2: 466 (1973).

ThaIlAND. Chiang Mai (Doi Inthanon N.P., Chiang Dao N.P.), Uthai Thani (Huay Kha Khaeng Wildlife Sanctuary). Wolseley \& Aguirre-Hudson (1997a: 333).

SPECIMENS. BM, CMU.

AsIA. China, India, Malaysia, Papua New Guinea, Sri Lanka.

Leptogium azureum (Sw. ex Ach.) Mont., in Webb \& Berthelot, Hist. Nat. Îles Canar. 3(2): 129 (1840).

ThailAND. Chiang Mai (Doi Suthep N.P., Chiang Dao N.P.), Lampang (Doi Khun Tan N.P.), Nakhon Nayok (Khao Yai N.P.), Uthai Thani (Huay Kha Khaeng Wildlife Sanctuary), Yala (Banglang Reservoir). Boonpragob et al. (1998: 217).

SPECIMENS. BM, CMU.

AsIA. China, India, Indonesia, Malaysia, Papua New Guinea, Sri Lanka, Vietnam. 
Leptogium burgessii (L.) Mont., in Webb \& Berthelot, Hist. Nat. Îles Canar. 3(2): 129 (1840).

ThaIland. Chiang Mai (Queen Sirikit Botanical Garden). Mongkonsuk et al. (1996: 17).

SPECIMEN. Queen Sirikit Botanical Garden Herbarium.

ASIA. India, Indonesia.

Leptogium caesium (Ach.) Vain., Acta Soc. Fauna Fl. Fenn. 7: 225 (1890).

ThaIl_And. Trat (Koh Chang I.). Vainio (1909: 116).

SPECIMEN. TUR-V.

AsIA. India, Indonesia, Papua New Guinea.

Leptogium chloromelum (Sw.) Nyl., Syn. Meth. Lich. 1: 128 (1858).

Thailand. Chiang Mai (Queen Sirikit Botanical Garden). Mongkonsuk et al. (1996: 17).

SPECIMEN. Queen Sirikit Botanical Garden Herbarium.

ASIA. India, Malaysia, Sri Lanka, Taiwan.

Leptogium cochleatum (Dicks.) P.M. Jørg. \& P. James, Lichenologist 15: 113 (1983).

Leptogium tremelloides auct.

Thailand. Chumphon (Koh Tao I.). Paulson (1930: 99, as Leptogium tremelloides).

SPECIMEN. BM.

AsIA. India.

Leptogium coralloideum (Meyen \& Flot.) Vain., Ann. Acad. Sci. Fenn., ser. A, 6(7): 110 (1915).

Leptogium chloromelum auct. non. (Sw.) Nyl.

Thailand. Chiang Mai (Doi Inthanon N.P.), Chumphon (Koh Tao I.). Uthai Thani (Huay Kha Khaeng Wildlife Sanctuary). Paulson (1930: 99, as Leptogium chloromelum auct. non. (Sw.) Nyl.).

SPECIMENS. BKF, BM, CMU.

AsIA. Indonesia, Papua New Guinea, Philippines.

Leptogium cyanescens (Rabenh.) Körb., Syst. Lich. Germ.: 420 (1855).

THAiland. Chiang Mai (Doi Suthep N.P., Doi Inthanon N.P., Chiang Dao N.P.), Lampang (Doi Khun Tan N.P.), Nakhon Nayok (Khao Yai N.P.), Tak (Ton Krabakyai N.P.), Uthai Thani (Huay Kha Khaeng Wildlife Sanctuary). Wolseley (1991: 236), Wolseley \& Aguirre-Hudson (1997a: 333, 1997b: 355), Boonpragob et al. (1998: 217).

SPECIMENS. BKF, BM, CMU.

AsIA. China (incl. Hong Kong), India, Indonesia, Malaysia, Papua New Guinea, Sri Lanka, Taiwan.

Leptogium denticulatum Nyl., Ann. Sci. Nat. Bot., sér. 5, 7: 302 (1867).

ThaIland. Chiang Mai (Doi Suthep N.P., Doi Inthanon N.P., Chiang Dao N.P.), Nakhon Nayok (Khao Yai N.P.), Uthai Thani (Huay Kha Khaeng Wildlife Sanctuary). Wolseley \& Aguirre-
Hudson (1997a: 333; 1997b: 355), Boonpragob et al. (1998: 217).

SPECIMENS. BKF, BM, CMU.

ASIA. China (incl. Hong Kong), India, Malaysia.

Leptogium javanicum Mont., Syll. Gen. Sp. Crypt.: 379 (1856).

ThaIland. Chiang Mai (Doi Suthep N.P., Chiang Dao N.P.), Uthai Thani (Huay Kha Khaeng Wildlife Sanctuary). Wolseley \& Aguirre-Hudson (1997a: 333).

SPECIMENS. BKF, BM, CMU.

ASIA. India, Indonesia, Malaysia, Papua New Guinea, Philippines.

Leptogium marginellum (Sw.) S.F. Gray, Nat. Arr. Brit. Pl. 1: 401 (1821).

ThAILAND. Chiang Mai (Doi Suthep N.P., Doi Inthanon N.P., Queen Sirikit Botanical Garden), Tak (Ton Krabakyai N.P.), Uthai Thani (Huay Kha Khaeng Wildlife Sanctuary). Wolseley (1991: 236), Mongkonsuk et al. (1996: 17).

SPECIMENS. BKF, BM, CMU, Queen Sirikit Botanical Garden Herbarium.

ASIA. India, Indonesia, Papua New Guinea, Philippines.

Leptogium moluccanum (Pers.) Vain., Étude Class. Lich. Brésil 1: 223 (1890).

ThaILAND. Chiang Mai (Doi Suthep). Vainio (1921: 46), Sato (1962: 2).

SPECIMENS. TUR-V, TNS.

ASIA. China, India, Indonesia, Papua New Guinea, Taiwan.

Leptogium resupinans Nyl., Ann. Sci. Nat. Bot., sér. 4, 15: $368(1861)$.

THAILAND. Chiang Mai (Doi Inthanon N.P.). Jørgensen (1997: 128).

SPECIMENS. BM.

AsIA. India.

Leptogium trichophorum Müll. Arg., Flora 72: 505 (1889).

Thalland. Chiang Mai (Doi Suthep N.P., Doi Inthanon N.P.). Vainio (1921: 46).

SPECIMENS. BM, CMU, TUR-V.

AsIA. China, India, Indonesia, Philippines, Taiwan.

Letrouitia Hafellner \& Bellem.; Letrouitiaceae

Letrouitia domingensis (Pers.) Hafellner \& Bellem., Nova Hedwigia 35: 281 (1982).

Thailand. Chiang Mai (Doi Suthep N.P., Doi Inthanon N.P., Chiang Dao N.P.), Lampang (Doi Khun Tan N.P.), Nakhon Ratchasima (Khao Yai N.P.), Uthai Thani (Huay Kha Khaeng Wildlife Sanctuary), Yala (Banglang N.P.). Wolseley et al. (1994: 122), Boonpragob et al. (1998: 217).

SPECIMENS. BM, CMU, RAMK. 
ASIA. India, Papua New Guinea, Philippines, Sri Lanka, Taiwan, Vietnam.

Letrouitia flavocrocea (Nyl.) Hafellner \& Bellem., Nova Hedwigia 35: 281 (1982).

ThaIland. Uthai Thani (Huay Kha Khaeng Wildlife Sanctuary), Yala (La Or Rung). Wolseley et al. (1994: 122).

SPECIMENS. BM, CMU.

ASIA. India, Philippines.

Letrouitia leprolyta (Nyl.) Hafellner, Nova Hedwigia 35: 693 (1981).

Thailand. Chumphon (N. of Pranburi), Lampang (Doi Luang), Uthai Thani (Huay Kha Khaeng Wildlife Sanctuary). Hafellner (1981: 694), Wolseley et al. (1994: 122), Wolseley \& AguirreHudson (1997a: 333, 339; 1997b; 355).

SPECIMENS. BM.

AsIA. India, Indonesia, Papua New Guinea, Sri Lanka.

Letrouitia subvulpina (Nyl.) Hafellner, Nova Hedwigia 35: 705 (1982).

THAILAND. Nakhon Ratchasima (Khao Yai N.P.), Uthai Thani (Huay Kha Khaeng Wildlife Sanctuary). Wolseley et al. (1994: 122), Wolseley \& Aguirre-Hudson (1997a: 333; 1997b: 355), Boonpragob et al. (1998; 217).

SPECIMENS. BM, RAMK.

AsIA. India, Indonesia, Papua New Guinea, Sri Lanka.

Letrouitia transgressa (Malme) Hafellner \& Bellem., Nova Hedwigia 35: 710 (1982).

THAILAND. Chiang Mai (Doi Inthanon N.P.), Lampang (Doi Luang), Uthai Thani (Huay Kha Khaeng Wildlife Sanctuary). Wolseley et al. (1994: 122).

SPECIMENS. BM, CMU.

ASIA. India, Indonesia, Taiwan.

Letrouitia vulpina (Tuck.) Hafellner \& Bellem., Nova Hedwigia 35: 281 (1982).

THAILAND. Satun (Thaleban N.P.), Uthai Thani (Huay Kha Khaeng Wildlife Sanctuary). Wolseley et al. (1994: 122), Wolseley \& AguirreHudson (1997a: 333, 339; 1997b: 355).

SPECIMENS. BM.

AsIA. India, Indonesia, Malaysia, Papua New Guinea, Philippines, Sri Lanka.

Lobaria (Schreb.) Hoffm.; Lobariaceae

Lobaria discolor (Bory) Hue, Nouv. Arch. Mus. Paris, sér. 4, 3: 23 (1901).

ThaIland. Chiang Mai (Chieng Dao N.P., Doi Inthanon N.P., Doi Suthep N.P.). Yoshimura (1978: 39), Wolseley (1991: 234).

SPECIMENS. BM, C.

ASIA. China, India, Indonesia, Philippines, Sri Lanka, Taiwan.
Lobaria isidiosa (Müll. Arg.) Vain., Philipp. J. Sci., sect. C, 8: 129 (1913).

ThaILAND. Chiang Mai (Doi Inthanon N.P., Doi Suthep N.P.). Sato (1962: 3), Yoshimura (1971: 295).

SPECIMENS. TNS.

AsIA. China, Indonesia, Malaysia, Papua New Guinea, Philippines, Sri Lanka, Taiwan, Vietnam.

Lobaria pseudopulmonaria Gyeln., Acta Fauna Fl. Univ., ser. 2, 1(5/6): 6 (1933).

ThaIlAnd. Chiang Mai (Chieng Dao N.P., Doi Suthep N.P.), Loei (Phu Luang N.P). Yoshimura (1971: 291; 1978: 39).

SPECIMENS. C, KYO, TNS.

AsIA. China, India, Indonesia, Malaysia, Papua New Guinea, Philippines, Taiwan.

Lobaria retigera (Bory) Trevis. Schedae Lich. Veneta No. 75 (1869).

ThaIland. Chiang Mai (Doi Inthanon N.P., Doi Suthep N.P.). Wolseley \& Aguirre-Hudson (1997a: 334).

SPECIMENS. BM.

AsIA. China, India, Indonesia, Philippines, Sri Lanka, Taiwan, Vietnam.

Maronea A. Massal; Fuscideaceae

Maronea constans (Nyl.) Hepp, Flecht. Eur: 771 (1860).

ThaIland. Chiang Mai (Doi Suthep N.P.). Wolseley \& AguirreHudson (1997a: 334).

SPECIMENS. BM.

AsIA. India, Philippines.

Mazosia A. Massal.; Opegraphaceae

Mazosia melanophthalma (Müll. Arg.) R. Sant., Symb. Bot. Upsal. 12(1): 117 (1952).

ThaILAND. Nakhon Ratchasima (Khao Yai N.P.). Boonpragob et al. (1998: 217).

SPECIMENS. RAMK.

ASIA. China (incl. Hong Kong), India, Indonesia, Malaysia, Papua New Guinea, Philippines, Sri Lanka, Vietnam.

Megalospora Meyen; Megalosporaceae

Megalospora bicolorata (Vain.) Zahlbr., Cat. Lich. Univ. 4: 87 (1927).

ThaIland. Chiang Mai (Doi Sutep N.P.). Vainio (1921: 48).

SPECIMEN. TUR-V (not located)

ASIA. -

Megalospora sulphurata Meyen, in Meyen \& Flotow, Nov. Actorum Acad. Caes. Leop.-Carol. Nat. Cur. 19, suppl.: 228 (1843). 
ThAILAND. Chiang Mai (Doi Suthep N.P.). Wolseley \& AguirreHudson (1997a: 339).

SPECIMENS. BM.

ASIA. India, Indonesia, Papua New Guinea.

Megalospora tuberculosa (Fée) Sipman, Biblioth.

Lichenol. 18: 156 (1983).

ThAILAND. Chiang Mai (Doi Suthep N.P.), Nakhon Ratchasima (Khao Yai N.P.). Sipman (1983: 238), Wolseley \& Aguirre-Hudson (1997a: 339), Boonpragob et al. (1998: 217).

SPECIMENS. BM, RAMK, US.

ASIA. China, India, Taiwan.

Menegazzia A. Massal.; Hypogymniaceae

Menegazzia terebrata (Hoffm.) A. Massal., Neag. Lich.: 3 (1854).

ThAILAND. Chiang Mai (Doi Inthanon N.P.). Wolseley \& AguirreHudson (1995: 323; 1997a: 339).

SPECIMEN. BM.

AsIA. China, India, Malaysia, Taiwan.

Microtheliopsis Müll. Arg.; Microtheliopsidaceae

Microtheliopsis uleana Müll. Arg., Flora 73: 195 (1890).

THAILAND. Nakhon Ratchasima (Khao Yai N.P.). Boonpragob et al. (1998: 217).

SPECIMENS. CANB.

AsIA. Indonesia, Malaysia, Papua New Guinea, Philippines, Sri Lanka.

Myelochroa (Asahina) Elix \& Hale; Parmeliaceae

Myelochroa aurulenta (Tuck.) Elix \& Hale, Mycotaxon 29: 240 (1987).

ThaIland. Chiang Mai (Doi Inthanon N.P.), Phitsanulok (Phu Hin Rongkla N.P.). Moon et al. (2000a: 102).

SPECIMENS. TNS.

ASIA. -

Myelochroa entotheiochroa (Hue) Elix \& Hale, Mycotaxon 29: 240 (1987).

ThaIland. Chiang Mai (Maetang). Moon et al. (2000a: 102). SPECIMENS. TNS.

ASIA. -

Myelochroa irrugens (Hue) Elix \& Hale, Mycotaxon 29: 241(1987).

Thailand. Chiang Mai (Maetang). Moon et al. (2000a: 102). SPECIMENS. TNS.

ASIA. -

Myelochroa perisidians (Nyl.) Elix \& Hale, Mycotaxon 29: 241 (1974).
Parmelina perisidians (Nyl.) Hale.

Thailand. Loei (Ban Sui). Hale (1976c: 39, as Parmelina perisidians), Moon et al. (2000a: 102).

SPECIMENS. TNS, US.

ASIA. Philippines, Sri Lanka.

Myelochroa siamea Kurok., J. Jap. Bot. 73: 13 (1998).

ThAILAND. Chiang Mai (Maetang District). Kurokawa (1998: 13), Moon et al. (2000a: 102).

SPECIMENS. BM, TNS.

ASIA. -

Myelochroa xantholepis (Mont. \& Bosch) Elix \& Hale, Mycotaxon 29: 241 (1987).

Parmelina xantholepis Mont. \& Bosch.

ThAildnd. Chiang Mai (Maetang, Doi Suthep N.P., Chiang Dao N.P.), Phitsanulok (Phu Hin Rongkla, N.P.), Uthai Thani (Huay Kha Khaeng Wildlife Sanctuary). Hale (1976c: 53, as Parmelina xantholepis), Moon et al. (2000a: 102).

SPECIMENS. BM, CMU, TNS, US.

ASIA. India, Indonesia, Philippines.

Myeloconis P.M. McCarthy \& Elix; Myeloconaceae

Myeloconis erumpens P.M. McCarthy \& Elix, Lichenologist 28: 406 (1996).

ThAILAND. Nakhon Ratchasima (Khao Yai N.P.). Boonpragob et al. (1998: 217).

SPECIMENS. CANB, RAMK.

AsIA. Papua New Guinea.

Myriotrema Fée; Thelotremataceae

Myriotrema albocinctum Hale, Bull. Brit. Mus. (Nat. Hist.), Bot. 8(3): 273(1981).

ThAILAND. Nakhon Ratchasima (Khao Yai N.P.). Boonpragob et al. (1998: 217).

SPECIMENS. BM, RAMK.

ASIA. Malaysia, Sri Lanka.

Myriotrema bahianum (Ach.) Hale, Mycotaxon 11: 132 (1980).

ThAILAND. Uthai Thani (Huay Kha Khaeng Wildlife Sanctuary). Homchantara (1999: 151-153).

SPECIMENS. BM, RAMK.

ASIA. Malaysia, Papua New Guinea, Philippines.

Myriotrema clandestinum (Fée) Hale, Mycotaxon 11: 133 (1980).

THAILAND. Nakhon Ratchasima (Khao Yai N.P.). Boonpragob et al. (1998: 217).

SPECIMENS. RAMK.

ASIA. Sri Lanka. 
Myriotrema compunctum (Ach.) Hale, Mycotaxon 11: 133 (1980).

ThAILAND. Lampang (Teak Improvement Centre), Rayong (Klaeng District). Homchantara (1999: 153).

SPECIMENS. BM, RAMK.

ASIA. Indonesia, Philippines, Sri Lanka, Vietnam.

Myriotrema costaricense (Müll. Arg.) Hale, Mycotaxon 11: 133 (1980).

THAILAND. Nakhon Ratchasima (Khao Yai N.P.), Uthai Thani (Huay Kha Khaeng Wildlife Sanctuary). Boonpragob et al. (1998: 217), Homchantara (1999: 156-158).

SPECIMENS. BM, RAMK.

ASIA. India, Malaysia.

Myriotrema decorticum Hale, Bull. Brit. Mus. (Nat. Hist.), Bot. 8(3): 277 (1981).

ThaILAND. Uthai Thani (Huay Kha Khaeng Wildlife Sanctuary). Homchantara (1999: 159-161).

SPECIMENS. BM.

ASIA. Sri Lanka.

Myriotrema desquamans (Müll. Arg.) Hale, Mycotaxon

11: 133 (1980).

ThAILAND. Chantaburi (Khitchakut N.P., Khao Soi Dao Wildlife Sanctuary), Nakhon Sri Thammerat (Khao Luang N.P.), Sukhothai (Ramkhamhaeng N.P.), Tak (Ton Krabak Yai N.P.). Homchanatara (1999: 161-163).

SPECIMENS. BM, RAMK.

AsIA. India, Malaysia, Papua New Guinea, Philippines, Sri Lanka.

Myriotrema fissurinum Hale, Bull. Brit. Mus. (Nat. Hist.), Bot. 8(3): 279 (1981).

THAILAND. Nakhon Ratchasima (Khao Yai N.P.), Phetchabun (Phu Hin Rongkla N.P.). Homchantara (1999: 159).

SPECIMENS. RAMK.

ASIA. India, Sri Lanka.

Myriotrema glaucescens (Nyl.) Hale, Mycotaxon 11: 133 (1980).

THAILAND. Chiang Mai (Doi Suthep N.P.), Uthai Thani (Huay Kha Khaeng Wildlife Sanctuary). Homchantara (1999: 168).

SPECIMENS. BM, RAMK.

ASIA. India, Sri Lanka.

Myriotrema grandisporum Homchantara \& Coppins, Lichenologist 34: (in press).

THAILAND. Nakhon Ratchasima (Khao Yai N.P.). Homchantara (1999: 224-225, as Myriotrema 'unidentified 10').

SPECIMENS. E (isotype), RAMK (holotype).

ASIA. -
Myriotrema khaoyaianum Homchantara \& Coppins,

Lichenologist 34: (in press).

ThAiland. Nakhon Ratchasima (Khao Yai N.P.). Homchantara (1999: 209-211, as Myriotrema 'unidentified 3').

SPECIMENS. E (isotype), RAMK (holotype).

ASIA. -

Myriotrema khuntanense Homchantara \& Coppins, Lichenologist 34: (in press).

THAILAND. Lampang (Doi Khuntan N.P.). Homchantara (1999: 226-227, as Myriotrema 'unidentified 11').

SPECIMENS. BM (holotype), RAMK (isotype).

ASIA.

Myriotrema masonhalei (Patw. \& C.R. Kulk.) Hale, Mycotaxon 11: 134 (1980).

THAILAND. Nakhon Ratchasima (Khao Yai N.P.). Boonpragob et al. (1998: 217).

SPECIMENS. RAMK.

ASIA. India.

Myriotrema microporum (Mont.) Hale, Mycotaxon 11: 134 (1980).

Thailand. Chiang Mai (Doi Suthep N.P.), Nakhon Ratchasima (Khao Yai N.P.), Phetchabun (Phu Hin Rongkla N.P.). Boonpragob et al. (1998: 217), Homchantara (1999: 175).

SPECIMENS. BM, RAMK.

ASIA. India, Indonesia, Malaysia, Philippines, Sri Lanka, Taiwan.

Myriotrema microstomum (Müll. Arg.) Hale, Mycotaxon

11: 134 (1980).

Thailand. Chiang Mai (Doi Suthep N.P.), Phetchabun (Phu Hin Rongkla N.P.), Sukhotai (Ramkhamhaeng N.P.). Homchantara (1999: 177).

SPECIMENS. BM, RAMK.

AsIA. Sri Lanka, Taiwan.

Myriotrema minutulum (Hale) Hale, Mycotaxon 11: 134 (1980).

THAILAND. Uthai Thani (Huay Kha Khaeng Wildlife Sanctuary). Homchantara (1999: 180).

SPECIMENS. BM.

ASIA. Sri Lanka.

Myriotrema minutum (Hale) Hale, Mycotaxon 11: 134 (1980).

THAiland. Nakhon Ratchasima (Khao Yai N.P.). Homchantara (1999: 182).

SPECIMEN. RAMK.

ASIA. Malaysia, Sri Lanka.

Myriotrema olivaceum Fée, Essai Crypt. Écorc. Offic.: 103 (1824). 
Thailand. Loei (Na Haeo N.P., Phuteen Suansai forest, Ban Bomoeng Noi). Homchantara (1999: 185).

SPECIMENS. RAMK.

AsIA. Sri Lanka.

Myriotrema phaeosporum (Nyl.) Hale, Mycotaxon 11: 134 (1980).

Leptotrema phaeosporum (Nyl.) Müll. Arg.

Thailand. Chumphon (Koh Tao I.). Paulson (1930: 99, as Leptotrema phaeosporumi).

SPECIMEN. BM (not located).

ASIA. -

Myriotrema polytretum Hale, Bull. Brit. Mus. (Nat. Hist.), Bot. 8(3): 291 (1981).

ThaIland, Chiang Mai (Doi Suthep N.P.), Lampang (Doi Pi Luang Ditrict), Loei (Phu Ruea N.P.), Uthai Thani (Huay Kha Khaeng N.P.). Homchantara (1999: 187).

SPECIMENS. BM, RAMK.

ASIA. Malaysia, Sri Lanka.

Myriotrema rugiferum (Harm.) Hale, Mycotaxon 11: 135 (1980).

Thailand. Chiang Mai (Doi Suthep N.P.), Phetchabun (Phu Hin Rongkla N.P.). Homchantara (1999: 189).

SPECIMENS. BM, RAMK.

ASIA. India, Papua New Guinea, Philippines, Sri Lanka.

Myriotrema subconforme (Nyl.) Hale, Mycotaxon 11: 135 (1980).

THAILAND. Nakhon Ratchasima (Khao Yai N.P.). Boonpragob et al. (1998: 217).

SPECIMENS. RAMK.

AsIA. India.

Myriotrema subgranulosum Homchantara \& Coppins, Lichenologist 34: (in press).

Thalland. Chiang Mai (Doi Inthanon N.P.), Loei (Na Po District). Homchantara (1999: 205-207, as Myriotrema 'unidentified 1').

SPECIMENS. BM (holotype), RAMK (isotype).

ASIA. -

Myriotrema subminutum Homchantara \& Coppins, Lichenologist 34: (in press).

ThaILAND. Nakhon Ratchasima (Khao Yao N.P.), Phetchabun (Phu Hin Rongkla N.P.), Uthai Thani (Huay Kha Khaeng Wildlife Sanctuary). Homchantara (1999: 215-217, as Myriotrema 'unidentified 6').

SPECIMENS. E (isotype), RAMK (holotype).

ASIA. -

Myriotrema terebratulum (Nyl.) Hale, Mycotaxon 11: 135 (1980).

Thalland. Chiang Mai (Doi Inthanon N.P.), Loei (Na Po District),
Nakhon Ratchasima (Khao Yai N.P.). Boonpragob et al. (1998: 217), Homchantara (1999: 196).

SPECIMENS. BM, RAMK.

ASIA. India.

Myriotrema viride Nagarkar \& Hale, Mycotaxon 35: 440 (1989).

Thalland. Sukhothai (Ramkhamhaeng N.P.), Uthai Thani (Huay Kha Khaeng Wildlife Sanctuary). Boonpragob et al. (1998: 217), Homchantara (1999: 199).

SPECIMENS. BM, RAMK.

AsIA. Malaysia.

Myriotrema wightii (Taylor) Hale, Mycotaxon 11: 135 (1980).

THAILAND. Chiang Mai (Doi Suthep N.P.), Kanchanaburi (Erawan N.P.), Uthai Thani (Huay Kha Khaeng Wildlife Sanctuary). Homchantara (1999: 201).

SPECIMENS. BM, RAMK.

AsıA. India, Malaysia, Philippines, Sri Lanka.

Nephroma Ach.; Nephromataceae

Nephroma helveticum Ach., Lichenogr. Universalis: 523 (1810).

Nephroma tropicum (Müll. Arg.) Zahlbr.

THAILAND. Chiang Mai (Doi Inthanon N.P., Doi Suthep N.P.). Wolseley \& Aguirre-Hudson (1995: 324; 1997a: 334, 339, as Nephroma tropicum).

SPECIMENS. BM, CMU.

AsIA. China, India, Indonesia, Malaysia, Papua New Guinea.

Nephromopsis Müll. Arg.; Parmeliaceae

Nephromopsis stracheyi (Church. Bab.) Müll. Arg., Flora 74: 374 (1891).

Nephromopsis ornata auct. non (Müll. Arg.) Hue

Thalland. Chiang Mai (Doi Suthep N.P), Loei (Phu Rua N.P.). Wolseley \& Aguirre-Hudson (1995: 324, as Nephromopsis ornata auct. non (Müll. Arg.) Hue).

SPECIMENS. BKF, BM, CMU.

AsIA. China, India, Malaysia, Taiwan.

Normandina (Nyl.) Vain.; incert. sed.

Normandina pulchella (Borrer) Nyl., Ann. Sci. Nat., Bot., sér. 4, 15: 382 (1861).

ThaIlAND. Chiang Mai (Doi Inthanon N.P.), Nakhon Ratchasima (Khao Yai N.P.). Wolseley \& Aguirre-Hudson (1997a: 334), Boonpragob et al. (1998: 217).

SPECIMENS. BM, RAMK.

AsIA. China, Malaysia, Papua New Guinea. 
Ocellularia Meyen; Thelotremataceae

Ocellularia albomaculata Hale, Bull. Brit. Mus. (Nat. Hist.) Bot. 8(3): 300 (1981).

ThaIland. Satun (Thalae Ban N.P.). Homchantara (1999: 237).

SPECIMENS. BM.

ASIA. Sri Lanka.

Ocellularia allosporoides (Nyl.) Patw. \& C.R. Kulk., Kavaka 5: 5 (1977).

ThaILAND. Lampang (Wiang Kosai N.P.), Chantaburi (Khitchakut N.P.), Kanchanaburi (Khao Lem N.P.), Nakhon Ratchasima (Khao Yai N.P.). Boonpragob et al. (1998: 217), Homchantara (1999: 239).

SPECIMENS. BM, RAMK.

ASIA. India.

Ocellularia arecae (Vain.) Hale, Mycotaxon 11: 136 (1980).

Thelotrema arecae Vain.

Thelotrema interpositum Nyl.

THAILAND. Chantaburi (Khitchakut N.P.), Loei (Na Haeo N.P.), Nakhon Ratchasima (Khao Yai N.P.), Phetchabun (Phu Hin Rongkla N.P.), Trat (Koh Chang I.). Vainio (1909: 119, as Thelotrema arecae), Salisbury (1971: 277, as T. interpositum), Hale (1981: 305), Boonpragob et al. (1998: 217), Homchantara (1999: 242).

SPECIMENS. RAMK, TUR-V (holotype).

AsIA. India, Malaysia, Philippines.

Ocellularia ascidioidea Hale, Bull. Brit. Mus. (Nat. Hist.), Bot. 8(3): 300 (1981).

THAILAND. Nakhon Ratchasima (Khao Yai N.P.), Phetchabun (Phu Hin Rongkla N.P.), Sukhotai (Ramkhamhaeng N.P.), Uthai Thani (Huay Kha Khaeng Wildlife Sanctuary). Boonpragob et al. (1998: 217), Homchantara (1999: 245).

SPECIMENS. BM, RAMK.

ASIA. Malaysia, Sri Lanka.

Ocellularia asiatica (Vain.) Hale, Hedwigia 46: 175 (1907).

Thelotrema asiaticum Vain.

ThaILAND. Nakhon Ratchasima (Khao Yai N.P.), Trat (Koh Chang I.). Vainio (1909: 120, as Thelotrema asiaticum), Patwardan \& Kulkarni (1977a: 10, as T. asiaticum), Boonpragob et al. (1998: 217).

SPECIMENS. RAMK, TUR-V (holotype).

ASIA. India.

Ocellularia chonestoma (Leight.) Zahlbr., Cat. Lich. Univ. 2: 586 (1923).

THAILAND. Kanchanaburi (Khao Lem N.P.). Homchantara (1999: 248).

SPECIMENS. RAMK.
ASIA. Malaysia, Philippines, Sri Lanka.

Ocellularia concolor Meyen \& Flot., Nov. Actorum Acad. Caes. Leop.-Carol. Nat. Cur. 19, suppl.: 230 (1843).

ThaIlAnd. Chantaburi (Khitchakut N.P.), Chiang Mai (Doi Suthep N.P.), Nakhon Ratchasima (Khao Yai N.P.), Phetchabun (Phu Hin Rongkla N.P.), Sukhothai ( Ramkhamhaeng N.P.). Boonpragob et al. (1998: 217), Homchantara (1999: 250).

SPECIMENS. BM, RAMK.

ASIA. India, Malaysia, Philippines.

Ocellularia conformis (Fée) Hale, Mycotaxon 11: 136 (1980).

Thailand. Phetchabun (Phu Hin Rongkla N.P), Homchantara (1999: 250).

SPECIMENS. RAMK.

ASIA. India.

Ocellularia crassa (Müll. Arg.) Hale, Mycotaxon 11: 136 (1980).

ThaIland. Phetchabun (Phu Hin Rongkla N.P). Homchantara (1999: 250).

SPECIMENS. RAMK.

ASIA. India.

Ocellularia diacida Hale, Mycotaxon 7: 378 (1978).

ThaILAND. Chantaburi (Kitchakut N.P., Namtok Phliu N.P., Khao Soi Dao Wildlife Sanctuary), Chiang Mai (Doi Inthanon N.P., Doi Suthep N.P.), Lampang (Doi Pae Luang), Loei (Na Haeo N.P., Phu Riea N.P.), Nakhon Ratchasima (Khae Yai N.P.), Phetchabun (Phu Hin Rongkla N.P), Sakon Nakhon (Phu Phan N.P.), Sukhothai (Ramkhamhaeng N.P.), Uthai Thani (Huay Kha Khaeng Wildlife Sanctuary). Boonpragob et al. (1998: 217), Homchantara (1999: 250).

SPECIMENS. BM, RAMK.

AsIA. India, Sri Lanka.

Ocellularia diospyrosis Homchantara \& Coppins, Lichenologist 34: (in press).

THAILAND. Tak (Ton Krabak Yai N.P.). Homchantara (1999: 314 315, as Ocellularia 'unidentified 1').

SPECIMENS. BM (holotype).

ASIA.

Ocellularia dolichotata (Nyl.) Zahlbr., Cat. Lich. Univ. 2: 589 (1923).

Thelotrema siamense Vain.

ThAILAND. Nakhon Ratchasima (Khao Yai N.P.), Trat (Koh Chang I.). Vainio (1907: 174; 1909: 120, as Thelotrema siamense), Hale (1981: 303), Sethy et al. (1987: 193), Boonpragob et al. (1998: 217), Homchantara (1999: 264).

SPECIMENS. BM, C (isolectotype), RAMK, TUR-V (lectotype).

AsıA. India, Malaysia, Papua New Guinea, Philippines, Singapore. 
Ocellularia emersa (Kremp.) Müll. Arg., Flora 69: 310 (1886).

THAILAND. Chanthaburi (Khitchakut N.P.), Loei (Phu Ruea N.P.), Nakhon Ratchasima (Khao Yai N.P.). Boonpragob et al. (1998: 217), Homchantara (1999: 267).

SPECIMENS. RAMK.

ASIA. Sri Lanka.

Ocellularia epitrypa (Nyl.) Hale, Mycotaxon 11: 136 (1980).

THAILAND. Nakhon Ratchasima (Khao Yai N.P.), Uthai Thani (Huay Kha Khaeng Wildlife Sanctuary). Boonpragob et al. (1998: 217). Homchantara (1999: 269).

SPECIMENS. BM, RAMK.

ASIA. India, Philippines, Sri Lanka.

Ocellularia eumorpha (Stirt.) Hale, Mycotaxon 11: 136 (1980).

Thalland. Chiang Mai (Doi Suthep N.P.), Loei (Na Haeo N.P.). Homchantara (1999: 271).

SPECIMENS. BM, RAMK.

ASIA. India, Sri Lanka.

Ocellularia fissa (Nyl.) Hale, Mycotaxon 11: 136 (1980).

Thalland. Nakhon Ratchasima (Khao Yai N.P.). Boonpragob et al. (1998: 217).

SPECIMENS. RAMK.

ASIA. India.

Ocellularia flavescens Homchantara \& Coppins,

Lichenologist 34: (in press).

THAILAND. Chiang Mai (Doi Inthanon N.P.). Homchantara (1999: 339-341, as Ocellularia 'unidentified 13').

SPECIMENS. BM (holotype), RAMK (isotype).

ASIA. -

Ocellularia groenhartii Hale, Mycotaxon 3: 173 (1975).

Thailand. Chantaburi (Khao Soi Dao N.P., Kitchakut N.P., Namtok Phliu N.P.), Kanchanaburi (Khao Laem N.P.), Lampang (Wiang Kosai N.P.), Nakhon Ratchasima (Khao Yai N.P.). Boonpragob et al. (1998: 217), Homchantara (1999: 272).

SPECIMENS. BM, RAMK.

ASIA. India, Indonesia.

Ocellularia guptei (Nagarkar, Sethy \& Patw.) D.D. Awasthi, Mycotaxon 23: 79 (1986).

ThAILAND. Chantaburi (Khitchakut N.P.), Nakhon Ratchasima (Khao Yai N.P.). Boonpragob et al. (1998: 217), Homchantara (1999: 276).

SPECIMENS. RAMK.

ASIA. India.

Ocellularia inthanonensis Homchantara \& Coppins, Lichenologist 34: (in press).
ThaILAND. Chiang Mai (Doi Inthanon N.P.). Homchantara (1999: 333-334, as Ocellularia 'unidentified 10').

SPECIMENS. BM (holotype), RAMK (isotype).

ASIA.

Ocellularia kansriae Homchantara \& Coppins, Lichenologist 34: (in press).

Thailand. Nakhon Ratchasima (Khao Yai N.P.), Pechabun (Phu Hin Rongkla N.P.), Sukhothai (Ramkhamhaeng N.P.). Homchantara (1999: 335-337, as Ocellularia 'unidentified 11').

SPECIMENS. E (isotype), RAMK (holotype).

ASIA. -

Ocellularia kratingensis Homchantara \& Coppins, Lichenologist 34: (in press).

Thalland. Chantaburi (Khitchakut N.P.), Nakhon Ratchasima (Khao Yai N.P.). Homchantara (1999: 316-318, as Ocellularia 'unidentified 2').

SPECIMENS. E (isotype), RAMK (holotype).

ASIA. Malaysia, Philippines, Sri Lanka.

Ocellularia leucina (Müll. Arg.) Hale, Mycotaxon 11: 137 (1980).

ThaILAND. Chantaburi (Namtok Phliu N.P.), Kanchanaburi (Kroengkawia), Nakhon Ratchasima (Khao Yai N.P.). Boonpragob et al. (1998: 217), Homchantara (1999: 278).

SPECIMENS. RAMK.

AsIA. India.

Ocellularia marivelensis (Vain.) Hale, Mycotaxon 11: 137 (1980).

ThAILAND. Nakhon Ratchasima (Khao Yai N.P.). Boonpragob et al. (1998: 217), Homchantara (1999: 281).

SPECIMENS. RAMK.

ASIA. India, Malaysia, Philippines.

Ocellularia massalongoi (Mont.) Hale, Mycotaxon 11: 137 (1980).

ThaILAND. Chantaburi (Namtok Phliu N.P.), Nakhon Ratchasima (Khao Yai N.P.), Uthai Thani (Huay Kha Khaeng Wildlife Sanctuary), Yala (Banlang N.P.). Boonpragob et al. (1998: 217), Homchantara (1999: 283).

SPECIMENS. BM, RAMK.

AsIA. India, Malaysia, Papua New Guinea, Philippines.

Ocellularia meiosperma (Nyl.) Hale, Mycotaxon 11: 137 (1980).

ThaIland. Chantaburi (Namtok Phliu N.P.), Chiang Mai (Doi Suthep N.P.), Nakhon Ratchasima (Khao Yai N.P.). Boonpragob et al. (1998: 217), Homchantara (1999: 281).

SPECIMENS. RAMK.

ASIA. India, Indonesia, Malaysia, Philippines, Sri Lanka. 
Ocellularia melanopthalma Homchantara \& Coppins, Lichenologist 34: (in press).

Thailand. Phetchabun (Phu Hin Rongkla N.P.). Homchantara (1999: 324-326, as Ocellularia 'unidentified 6').

SPECIMENS. E (isotype), RAMK (holotype).

ASIA. -

Ocellularia monosporoides (Nyl.) Hale, Mycotaxon 11: 137 (1980).

ThaILAND. Chantaburi (Khitchakut N.P.), Phetchabun (Phu Hin Rongkla N.P.). Homchantara (1999: 288-290).

SPECIMENS. RAMK.

AsIA. Sri Lanka.

Ocellularia microascidium (Vain.) Zahlbr., Cat. Lich.

Univ. 2: 595 (1923).

Thelotrema microascidium Vain.

Thalland. Trat (Koh Kong I.). Vainio (1907: 176, as Thelotrema microascidium).

SPECIMEN. TUR-V (holotype).

ASIA. -

Ocellularia neoleucina Homchantara \& Coppins,

Lichenologist 34: (in press).

THAILAND. Chantaburi (Namtok Phliu N.P.). Homchantara (1999: 333-334, as Ocellularia 'unidentified 15').

SPECIMENS. E (isotype), RAMK (holotype).

ASIA. -

Ocellularia neopertusariiformis Hale, Bull. Brit. Mus. (Nat. Hist.), Bot. 8(3): 315 (1981).

Thailand. Loei (Phu Ruea N.P.), Nakhon Ratchasima (Khao Yai N.P.), Phetchabun (Phu Hin Rongkla N.P.). Homchantara (1999: 290-293).

SPECIMENS. RAMK.

ASIA. Malaysia, Sri Lanka.

Ocellularia nylanderiana Hale, Phytologia 26: 414 (1973).

Thalland. Nakhon Ratchasima (Khao Yai N.P.). Boonpragob et al. (1998: 217).

SPECIMEN. RAMK.

AsIA. India.

Ocellularia paleoamplior Aptroot \& Sipman, J. Hattori Bot. Lab. 91: 333 (2001).

THAILAND. Nakhon Ratchasima (Khao Yai N.P.). Homchantara (1999: 326-328, as Ocellularia 'unidentified 7').

SPECIMENS. RAMK (holotype).

ASIA. -

Ocellularia papillata (Leight.) Zahlbr., Cat. Lich. Univ. 2: 597 (1923).
THAILAND. Chantaburi (Namtok Phliu N.P., Khitchakut N.P.), Lampang (Doi Mae Luang), Nakhon Ratchasima (Khao Yai N.P.), Sakhon Nakhon (Phu Phan N.P.), Sukhothai (Ramkhamhaeng N.P.), Uthai Thani (Huay Kha Khaeng Wildlife Sanctuary). Boonpragob et al. (1998: 217), Homchantara (1999: 295).

SPECIMENS. BM, RAMK.

ASIA. India, Philippines, Sri Lanka.

Ocellularia peremergens Homchantara \& Coppins, Lichenologist 34: (in press).

Thalland. Chiang Mai (Doi Inthanon N.P., Doi Suthep N.P.), Loei (Na Haeo N.P.), Nakhon Ratchasima (Khao Yai N.P.). Homchantara (1999: 331-332, 337, 349-350, as Ocellularia 'unidentified 9', '12', '18').

SPECIMENS. BM (holotype), RAMK (isotype).

ASIA. -

Ocellularia perforata (Leight.) Müll. Arg., Hedwigia 31: 284 (1892).

ThAILAND. Chantaburi (Khao Soi Dao Wildlife Sanctuary, Namtok Phliu N.P., Kitchakut N.P.), Nakhon Ratchasima (Khao Yai N.P.), Nakhon Sri Thammerat (Khao Luang N.P.), Petchabun (Phu Hin Rongkla N.P.), Sukhothai (Ramkhamhaeng N.P.). Boonpragob et al. (1998: 217), Homchantara (1999: 297).

SPECIMENS. RAMK.

AsIA. China (Hong Kong), India, Malaysia, Papua New Guinea.

Ocellularia pertusariformis (Leight.) Zahlbr., Cat. Lich. Univ. 2: 598 (1923).

ThAILAND. Nakhon Ratchasima (Khao Yai N.P.). Boonpragob et al. (1998: 217).

SPECIMENS. RAMK.

ASIA. India.

Ocellularia pluripora Hale, Bull. Brit. Mus. (Nat. Hist.), Bot. 8(3): 319 (1981).

THAIlAND. Chanthburi (Khitchakut N.P., Namtok Phliu N.P.), Loei (Phu Ruea N.P., Na Haeo N.P.), Nakhon Ratchasima (Khao Yai N.P.). Boonpragob et al. (1998: 217), Homchantara (1999: 304).

SPECIMENS. RAMK.

AsIA. India, Malaysia.

Ocellularia pluripoides Homchantara \& Coppins,

Lichenologist 34: (in press).

ThaILAND. Chiang Mai (Doi Suthep N.P.). Homchantara (1999: 318-319, as Ocellularia 'unidentified 3').

SPECIMENS. BM (holotype).

ASIA. -

Ocellularia punctulata (Leight.) Zahlbr., Cat. Lich. Univ. 2. 599 (1923).

THAILAND. Nakhon Ratchasima (Khao Yai N.P.). Boonpragob et al. (1998: 217).

SPECIMENS. RAMK.

ASIA. India, Malaysia. 
Ocellularia rhicnoporoides Homchantara \& Coppins, Lichenologist 34: (in press).

THAILAND. Chantaburi (Namtok Phliu N.P.). Homchantara (1999: 329-330, as Ocellularia 'unidentified 8').

SPECIMENS. E (isotype), RAMK (holotype).

ASIA. -

Ocellularia sticticans Hale, Bull. Brit. Mus. (Nat. Hist.), Bot. 8(3): 323 (1981).

THAILAND. Nakhon Ratchasima (Khao Yai N.P.). Boonpragob et al. (1998: 217).

SPECIMENS. RAMK.

ASIA. Sri Lanka.

Ocellularia subleucina Homchantara \& Coppins,

Lichenologist 34: (in press).

THAILAND. Chantaburi (Khitchakut N.P.). Homchantara (1999: 341-343, as Ocellularia 'unidentified 14').

SPECIMENS. E (isotype), RAMK (holotype).

ASIA. -

Ocellularia wolsleyanum Homchantara \& Coppins,

Lichenologist 34: (in press).

ThAILAND. Nakhon Ratchasima (Khao Yai N.P.). Homchantara (1999: 347-349, as Ocellularia 'unidentified 17').

SPECIMENS. E (isotype), RAMK (holotype).

ASIA. -

Opegrapha Ach.; Opegraphaceae

Opegrapha aff. lambinonii Sérus., Lejeunia 90: 2 (1978).

THAILAND. Nakhon Ratchasima (Khao Yai N.P.). Sérusiaux (1985: 23).

SPECIMEN. LG.

ASIA. India, Papua New Guinea, Philippines, Sri Lanka.

Opegrapha prosodea Ach., Methodus: 22 (1803).

Thalland. Chumphon (Koh Tao I.). Paulson (1930: 99).

SPECIMEN. BM.

ASIA. China, India, Malaysia, Papua New Guinea, Taiwan.

Opegrapha robusta Vain., Bot. Tidsskr. 29: 137 (1909).

Thalland. Trat (Koh Chang I.). Vainio (1909: 137).

SPECIMENS. TUR-V (syntypes).

ASIA. Indonesia, Papua New Guinea.

Opegrapha subvulgata Nyl., Flora 52: 71 (1869).

ThaILAND. Trat (Koh Chang I.). Vainio (1909: 136).

SPECIMEN. TUR-V.

ASIA. India.
Pannaria Delise ex Bory; Pannariaceae

Pannaria dispartita (Nyl) Vain., Suomal. Elain-ja Kasvit. Seuran Vanamon Julk. 1(3): 45 (1921).

THAILAND. Chiang Mai (Doi Suthep N.P.). Vainio (1921: 45).

SPECIMEN. TUR-V.

ASIA. -

Paraparmelia Elix \& J. Johnst.; Parmeliaceae

Paraparmelia rodriguesiana (Hue) Elix \& J. Johnst., Mycotaxon 27: 280 (1986).

Thalland. Phitsanulok (Phu Hin Rongkla, N.P.). Moon et al. (2000a: 102).

SPECIMEN. TNS.

ASIA. -

Parmelaria D.D. Awasthi; Parmeliaceae

Parmelaria thomsonii (Stirt.) D.D. Awasthi, J. Hattori Bot. Lab. 63: 368 (1987).

Thailand. Chiang Mai (Doi Suthep N.P.), Loei (Phu Rua N.P.). Wolseley \& Aguirre-Hudson (1995: 325).

SPECIMENS. BM, DUKE, TNS.

ASIA. India, Nepal.

Parmelia Ach.; Parmeliaceae

Parmelia saxatilis (L.) Ach., Methodus: 204 (1803).

THAILAND. Chiang Mai (Queen Sirikit Botanical Garden), Mongkonsuk et al. (1996: 17).

SPECIMEN. Queen Sirikit Botanical Garden Herbarium.

ASIA. China, India.

Parmeliella Müll. Arg.; Pannariaceae

Parmeliella brisbanensis (C. Knight) P.M. Jørg. \& D.J. Galloway, Fl. Australia 54: 316 (1992).

THAIland. Chiang Mai (Doi Suthep N.P.), Nakhon Ratchasima (Khao Yai N.P.), Uthai Thani (Huay Kha Khaeng Wildlife Sanctuary). Wolseley \& Aguirre-Hudson (1995: 325; 1997a: 334, 1997b: 356), Boonpragob et al. (1998: 217).

SPECIMENS. BM, RAMK.

ASIA. -

Parmeliella papillata P.M. Jørg., Biblioth. Lichenol. 78: 133 (2001).

THAIlAND. Uthai Thani (Huay Kha Khaeng Wildlife Sanctuary). Jørgensen (2001: 134).

SPECIMENS. BM.

ASIA. Papua New Guinea. 
Parmelina Elix; Parmeliaceae

Parmelina quercina (Willd.) Hale, Phytologia 28: 483 (1974).

ThaILAND. Chiang Mai (Queen Sirikit Botanical Garden). Mongkonsuk et al. (1996: 17).

SPECIMEN. Queen Sirikit Botanical Garden Herbarium.

AsIA. China, India.

Parmelina usambarensis (J. Steiner \& Zahlbr.) Hale, Phytologia 28: 483 (1974).

Parmelia usambarensis J. Steiner \& Zahlbr.

Thailand. Loei (near Phu Kradung). Hale (1976c: 51, as Parmelia usambarensis), Singh (1979: 222, as P. usambarensis), Kurokawa \& Kashiwadani (1988: 7, as P. usambarensis), Moon et al. (2000a: 102).

SPECIMENS. TNS.

AsIA. India.

Parmelinella Elix \& Hale; Parmeliaceae

Parmelinella chozoubae (Singh \& Sinha) Elix \&

Pooprang, in Pooprang et al., Mycotaxon 71: 121 (1999).

Thailand. Chiang Mai (Doi Suthep N.P.), Loei (Nahaew N.P.), Nakhon Ratchasima (Khao Yai N.P.), Phitsanulok (Phu Hin Rongkla N.P.). Pooprang et al. (1999: 121).

SPECIMENS. RAMK.

ASIA. India.

Parmelinella simplicior (Hale) Elix \& Hale, Mycotaxon 29: 242 (1987).

Thailand. Phitsanulok (Phu Hin Rongkla N.P.). Moon et al. (2000a: 102).

SPECIMENS. TNS.

ASIA. India.

Parmelinella wallichiana (Taylor) Elix \& Hale, Mycotaxon 29: 242 (1987).

Thailand. Chiang Mai (Doi Suthep N.P., Doi Inthanon N.P., Chiang Dao N.P., Doi Pacho, Maetang), Loei (Ban Sui, Phu Kradung), Phitsanulok (Phu Hin Rongkla, N.P.), Tak (Ton Krabakyai N.P.), Uthai Thani ( Huay Kha Khaeng Wildlife Sanctuary). Wolseley \& Aguirre-Hudson (1995: 326; 1997a: 334; 1997b: 356), Moon et al. (2000a: 103).

SPECIMENS. BKF, BM, CMU, TNS.

AsIA. China (incl. Hong Kong), India, Indonesia, Malaysia, Philippines, Sri Lanka, Taiwan.

Parmelinopsis Elix \& Hale; Parmeliaceae

Parmelinopsis expallida (Kurok.) Elix \& Hale, Mycotaxon 29: 242 (1987).

Parmelina expallida (Kurok.) Elix \& Hale.

ThaILAND. Chiang Mai (Maetang District). Hale (1976c: 30 as Parmelina expallida), Patwardhan \& Prabhu (1977: 111, as
P. expallida), Wolseley \& Aguirre-Hudson (1995: 325), Moon et al. (2000a: 103).

SPECIMENS. TNS, US.

AsIA. India, Taiwan.

Parmelinopsis horrescens (Taylor) Elix \& Hale, Mycotaxon 29: 242 (1987).

Thalland. Phitsanulok (Phu Hin Rongkla N.P.). Pooprang et al. (1999: 121).

SPECIMENS. RAMK.

ASIA. India, Sri Lanka.

Parmelinopsis microlobulata (D.D. Awasthi) Elix \& Hale, Mycotaxon 29: 242 (1987).

Thailand. Nakhon Sri Thammarat (Khao Luang N.P.), Phitsanulok (Phu Hin Rongkla N.P.). Pooprang et al. (1999: 121).

SPECIMENS. RAMK.

ASIA. India.

Parmelinopsis minarum (Vain.) Elix \& Hale, Mycotaxon 29: 243 (1987).

Thailand. Chiang Mai (Doi Suthep N.P.). Pooprang et al. (1999: 122).

SPECIMENS. RAMK.

ASIA. -

Parmeliopsis (Nyl. ex Stizenb.) Nyl.; Parmeliaceae

Parmeliopsis ambigua (Wulfen) Nyl., Lich. Lapp. Orient.: 121 (1866).

THAiland. Lampang (Khuntan N.P.). Pooprang et al. (1999: 122). SPECIMENS. RAMK.

ASIA. Nepal.

Parmotrema A. Massal.; Parmeliaceae

Parmotrema abessinicum (Kremp.) Hale, Phytologia 28: 334 (1974).

Thalland. Chiang Mai (Queen Sirikit Botanical Garden). Pooprang et al. (1999: 122).

SPECIMENS. RAMK.

ASIA. India, Malaysia.

Parmotrema amaniense (J. Steiner \& Zahlbr.) Krog \& Swinscow, Lichenologist 15: 129 (1983).

THAILAND. Uthai Thani (Huay Kha Khaeng Wildlife Sanctuary). Wolseley \& Aguirre-Hudson (1997a: 334).

SPECIMENS. BKF, BM.

ASIA. India.

Parmotrema andinum (Müll. Arg.) Hale, Phytologia 28: 334 (1974).

Parnelia andina Müll. Arg. 
Thailand, Chiang Mai (Doi Suthep, Chiang Dao N.P.). Hale (1965a: 236, as Parmelia andina).

SPECIMENS. BM, CMU, TNS.

AsIA. India, Malaysia.

Parmotrema austrosinense (Zahlbr.) Hale, Phytologia 28: 335 (1974).

Thailand. Phitsanulok (Lan Hin Taek, Phu Hin Rongkla N.P.). Moon et al. (2000a: 103).

SPECIMENS. TNS.

AsIA. China.

Parmotrema chinense (Osbeck) Hale \& Ahti, Taxon 35: 133 (1986).

Parmelia perlata Ach.

ThaIland. Chiang Mai (Doi Suthep N.P., Doi Inthanon N.P., Chiang Dao N.P.), Trat (Koh Chang I.). Vainio (1909: 104, as Parmelia perlata Ach.).

SPECIMENS. BM, CMU.

AsIA. China, Malaysia, Papua New Guinea, Philippines, Sri Lanka, Taiwan.

Parmotrema corniculans (Nyl.) Hale, Phytologia 28: 335 (1974).

ThaIland. Chiang Mai (Doi Inthanon N.P.). Pooprang et al. (1999: 122).

SPECIMEN. RAMK

AsIA. Indonesia, Laos, Malaysia, Papua New Guinea, Philippines.

Parmotrema crinitum (Ach.) M. Choisy, Bull. Mens. Soc. Linn. Soc. Bot. Lyon 21: 175 (1952).

ThaILAND. Chiang Mai (Queen Sirikit Botanical Garden). Mongkonsuk et al. (1996: 17).

SPECIMEN. Queen Sirikit Botanical Garden Herbarium.

ASIA. China, India, Papua New Guinea, Sri Lanka, Taiwan.

Parmotrema cristiferum (Taylor) Hale, Phytologia 28: 335 (1974).

Parmelia andina Taylor.

ThaIland. Chiang Mai (Chiang Dao N.P., Doi Suthep N.P.), Nakhon Nayok (Khao Yai N.P.), Udaun, Uthai Thani (Huay Kha Khaeng Wildlife Sanctuary). Hale (1965b: 241, as Parmelia andina), Boonpragob et al. (1999: 217), Moon et al. (2000a: 103).

SPECIMENS. BM, TNS, US.

ASIA. China, India, Indonesia, Malaysia, Myanmar, Papua New Guinea, Philippines, Sri Lanka, Taiwan.

Parmotrema dilatatum (Vain.) Hale, Phytologia 28: 335 (1974).

ThaILAND. Chiang Mai (Doi Suthep N.P., Queen Sirikit Botanical Garden), Nakhon Ratchasima (Khao Yai N.P). Mongkonsuk et al. (1996: 17), Boonpragob et al. (1999: 217), Moon et al. (2000a: 103).
SPECIMENS. RAMK, TNS, Queen Sirikit Botanical Garden Herbarium.

AsıA. India, Indonesia, Malaysia, Papua New Guinea, Sri Lanka.

Parmotrema dominicanum (Vain.) Hale, Phytologia 28: 335 (1974).

Thailand. Loei (Phu Kradung). Moon et al. (2000a: 103).

SPECIMENS. TNS.

ASIA. -

Parmotrema elacinatulum (Kurok.) Streimann, Biblioth. Lichenol. 22: 94 (1986).

ThAILAND. Nakhon Ratchasima (Khao Yai N.P.). Pooprang et al. (1999: 123)

SPECIMEN. RAMK.

ASIA. Papua New Guinea.

Parmotrema eunetum (Stirt.) Hale, Phytologia 28: 336 (1974).

Thalland. Chiang Mai (Doi Suthep N.P., Maetang). Moon et al. (2000a: 103).

SPECIMENS. TNS.

ASIA. Sri Lanka.

Parmotrema eurysacum (Hue) Hale, Phytologia 28: 336 (1974).

ThaIland. Chiang Mai (Queen Sirikit Botanical Garden). Mongkonsuk et al. (1996: 17).

SPECIMEN. Queen Sirikit Botanical Garden Herbarium.

ASIA.

Parmotrema explanatum (Hale) Hale, Phytologia 28: 336 (1974).

Parmelia explanata Hale.

Thailand. Loei (Phu Kradung). Hale (1965b: 199, as Parmelia explanata), Moon et al. (2000a: 103).

SPECIMENS. TNS (holotype), US (isotype).

ASIA. -

Parmotrema gardneri (C.W. Dodge) Sérus., Bryologist 87: 5 (1984).

Parmelia gardneri C.W. Dodge.

ThaILAND. Chiang Mai (Doi Inthanon N.P.), Trat (Koh Chang I.), Uthai Thani (Huay Kha Khaeng Wildlife Sanctuary). Vainio (1909: 105, as Parmelia gardneri), Wolseley \& Aguirre-Hudson (1997a: 334; 1997b: 355).

SPECIMENS. BM, TUR-V.

AsIA. Papua New Guinea.

Parmotrema hababianum (Gyeln.) Hale, Phytologia 28: 336 (1974). 
ThaIland. Chiang Mai (Queen Sirikit Botanical Garden). Pooprang et al. (1999: 125).

SPECIMEN, RAMK.

ASIA. China, India, Sri Lanka.

Parmotrema hypotropum (Nyl.) Hale, Phytologia 28: 337 (1974).

ThaIlAnd. Chiang Mai (Queen Sirikit Botanical Garden). Mongkonsuk et al. (1996: 17).

SPECIMEN. RAMK.

ASIA. -

Parmotrema incrassatum Hale ex DePriest \& B.W. Hale, Mycotaxon 67: 207 (1998).

ThaIland. Chiang Mai (Queen Sirikit Botanical Garden). Pooprang et al. (1999: 123).

SPECIMENS. Queen Sirikit Botanical Garden Herbarium.

AsIA. China (incl. Hong Kong).

Parmotrema lobulascens (J. Steiner) Hale, Phytologia 28: 337 (1974).

THAILAND. Chiang Mai (Doi Inthanon N.P.), Nakhon Nayok (Khao Yai N.P.), Uthai Thani (Huay Kha Khaeng Wildlife Sanctuary). Boonpragob et al. (1999: 217).

SPECIMEN. BM, RAMK.

ASIA. -

Parmotrema maclayanum (Müll. Arg.) Hale, Phytologia 28: 337 (1974).

Parmelia breviciliata Hale.

Thalland. Chiang Mai (Doi Suthep N.P., Doi Inthanon N.P., Queen Sirikit Botanical Garden), Loei (Ban Sui, Phu Kradung, Phu Luang), Uthai Thani (Huay Kha Khaeng Wildlife Sanctuary). Kurokawa \& Kashiwadani (1988: 4, as Parmelia breviciliata), Moon et al. (2000a: 103)

SPECIMENS. BM, TNS.

AsIA. Papua New Guinea.

Parmotrema mellissii (C.W. Dodge) Hale, Phytologia 28: 337 (1974).

Thailand. Loei (Phu Kradung). Moon et al. (2000a: 103).

SPECIMENS. TNS.

AsIA. China, India, Papua New Guinea, Taiwan.

Parmotrema nanfongense (Kurok.) DePriest \& B.W. Hale, Mycotaxon 67: 204 (1998).

ThAILAND. Chiang Mai (Doi Suthep N.P.). Moon et al. (2000a: 104).

SPECIMEN. TNS.

AsıA. Papua New Guinea, Taiwan.

Parmotrema nilgherrense (Nyl.) Hale, Phytologia 28: 338 (1974).
Parmelia nilgherrensis Nyl.

Parmelia siamensis Vain.

Thalland, Chiang Mai (Doi Suthep N.P., Doi Inthanon N.P.), Lampang (Doi Khun Tan N.P.), Nakhon Nayok (Khao Yai N.P.), Tak (Ton Krabakyai N.P.), Yala (Banglang Reservoir). Vainio (1921: 37 , as Parmelia siamensis), Hale (1965a: 333, as $P$. nilgherrensis), Wolseley \& Aguirre-Hudson (1997a: 334).

SPECIMENS. BM, TUR-V (holotype of Parmelia siamensis).

ASIA. China, India, Papua New Guinea, Philippines, Sri Lanka.

Parmotrema overeemii (Zahlbr.) Elix, Australas. Lichenol. 42: 23 (1998).

Thalland. Chiang Mai, Lampang (Doi Khun Tan N.P.), Loei (Phu Kradung N.P.), Nakhon Ratchasima (Khao Yai N.P.), Uthai Thani (Huay Kha Khaeng Wildlife Sanctuary). Pooprang et al. (1999: 123).

SPECIMENS. BKF, BM, CMU, RAMK, TNS, TUR-V.

AsIA. Indonesia, Papua New Guinea.

Parmotrema pancheri (Hue) Hale, Phytologia 28: 338 (1974).

Parmelia pancheri Hue.

Thalland. Chiang Mai (Doi Suthep). Hale (1965b: 236, as Parmelia pancheri), Moon et al. (2000a: 104).

SPECIMENS. TNS, US.

ASIA. -

Parmotrema permutatum (Stirt.) Hale, Phytologia 28: 338 (1974).

Parmelia permutata Stirt.

Thailand. Chiang Mai (Queen Sirikit Botanical Garden). Hale (1965b: 302, as Parmelia permutata), Kashiwadani (1975: 81), Moon et al. (2000a: 104).

SPECIMEN. TNS.

ASIA. India, Indonesia, Malaysia, Papua New Guinea.

Parmotrema planatilobatum (Hale) Hale, Phytologia 28: 338 (1974).

Thailand. Nakhon Ratchasima (Khao Yai N.P.). Pooprang et al. (1999: 124).

SPECIMEN. RAMK.

ASIA. India, Indonesia, Malaysia, Sri Lanka.

Parmotrema platyphyllinum (Vain.) Elix, Australas. Lichenol. 42: 23 (1998).

Parmelia platyphyllina Vain.

Parmelia zollingeri auct. non Hepp.

Thailand. Phitsanulok (Lan Hin Taek), Trat (Koh Chang I.). Vainio (1909: 106, as Parmelia platyphyllina), Hale (1965b: 267, as P. zollingeri), Moon et al. (2000a: 104).

SPECIMENS. TNS, TUR-V.

ASIA. - 
Parmotrema poolii (C.W. Dodge) Krog \& Swinscow, Lichenologist 15: 130 (1983).

ThaIland. Chiang Mai (Queen Sirikit Botanical Garden), Phitsanulok (Lan Hin Taek). Moon et al. (2000a: 104).

SPECIMENS. TNS.

ASIA. Papua New Guinea.

Parmotrema praesorediosum (Nyl.) Hale, Phytologia 28: 338 (1974).

ThaIland. Chiang Mai (Doi Saget, Maesa, Doi Suthep N.P., Queen Sirikit Botanical Garden), Songkla. David et al. (1990: 1299), Mongkonsuk et al. (1996: 17), Moon et al. (2000a: 104)

Specimens. CANB, TNS, US, Queen Sirikit Botanical Garden Herbarium.

AsIA. China (incl. Hong Kong), India, Malaysia, Papua New Guinea, Singapore, Sri Lanka, Taiwan.

Parmotrema procerum (J. Steiner \& Zahlbr.) Hale, Phytologia 28: 338 (1974).

Parmelia procera J. Steiner \& Zahlbr.

ThaILAND. Chiang Mai (Doi Suthep N.P.). Hale (1965b: 303, as Parmelia procera).

SPECIMENS. TNS, US.

ASIA. -

Parmotrema pseudocrinitum (des Abbayes) Hale, Phytologia 28: 338 (1974).

THAiland. Loei (near Phu Kradung). Kurokawa \& Kashiwadani (1988: 6), Moon et al. (2000a: 104).

SPECIMEN. TNS.

ASIA. -

Parmotrema pseudonilgherrense (Asahina) Hale, Mycotaxon 5: 441 (1977).

Thailand. Chiang Mai (Queen Sirikit Botanical Garden). Pooprang et al. (1999: 124).

SPECIMEN. RAMK.

AsIA. China, India, Nepal.

Parmotrema rampoddense (Nyl.) Hale, Phytologia 28: 338 (1974).

THAILAND. Chiang Mai (Doi Suthep N.P., Queen Sirikit Botanical Garden, Maetang), Loei (Phu Kradung N.P.), Phitsanulok (Lan Hin Taek, Phu Hin Rongkla, N.P.), Kurokawa \& Kashiwadani (1988: 6), Mongkonsuk et al. (1996: 17), Moon et al. (2000a: 104).

SPECIMENS. BM, TNS.

ASIA. China, India, Indonesia, Papua New Guinea, Sri Lanka, Taiwan.

Parmotrema robustum (Degel.) Hale, Phytologia 28: 338 (1974).

ThaIland. Chiang Mai (Queen Sirikit Botanical Garden),
Phitsanulok (Lan Hin Taek, Phu Hin Rongkla, N.P.). Mongkonsuk et al. (1996: 17), Moon et al. (2000a: 104).

SPECIMENS. TNS, Queen Sirikit Botanical Garden Herbarium.

ASIA. India, Papua New Guinea.

Parmotrema rubromarginatum Elix \& Pooprang, in Pooprang et al., Mycotaxon 72: 115 (1999).

Thailand. Loei, Nakhon Ratchasima. Pooprang et al. (1999: 115).

SPECIMENS. CANB (isotype), RAMK (incl. holotype).

ASIA. -

Parmotrema saccatilobum (Taylor) Hale, Phytologia 28: 339 (1974).

Thailand. Phuket (Ban Pae). Moon et al. (2000a: 104).

SPECIMEN. TNS.

ASIA. China, India, Papua New Guinea, Singapore, Taiwan.

Parmotrema sancti-angeli (Lynge) Hale, Phytologia 28: 339 (1974).

Parmelia sancti-angeli Lynge.

Thailand. Chiang Mai (Doi Suthep N.P., Doi Inthanon N.P., Doi Chieng Dao N.P., Maetang), Loei (near Phu Kradung), Phitsanulok (Lan Hin Taek, Phu Hin Rongkla, N.P.), Uthai Thani (Huay Kha Khaeng Wildlife Sanctuary). Hale (1965b: 307, as Parmelia sanctiangeli), Moon et al. (2000a: 104).

SPECIMENS. BM, TNS, US.

ASIA. China, India, Indonesia, Laos, Papua New Guinea, Sri Lanka, Taiwan.

Parmotrema stuppeum (Taylor) Hale, Phytologia 28: 339 (1974).

Parmelia perforata f. claudelii (Harm.) Vain.

Parmelia maxima Hue.

Thailand. Chiang Mai (Doi Suthep N.P.), Phuket (Ban Pae), Trat (Koh Chang I.). Vainio (1909: 105, as Parmelia perforataf. claudelii), des Abbayes (1964, as P. maxima), Moon et al. (2000a: 104).

SPECIMENS. TNS, TUR-V.

ASIA. India.

Parmotrema subarnoldii (des Abbayes) Hale, Phytologia 28: 339 (1974).

Thailand. Loei, Nakhon Ratchasima. Pooprang et al. (1999: 124)

SPECIMENS. RAMK.

AsIA. India, Indonesia, Papua New Guinea.

Parmotrema submarginale (Michx.) DePriest \& B.W. Hale, Mycotaxon 67: 208 (1998).

Parmotrema michauxianum (Zahlbr.) Hale.

ThaIland. Chiang Mai (Queen Sirikit Botanical Garden). Mongkonsuk et al. (1996: 17). 
SPECIMENS. Queen Sirikit Botanical Garden Herbarium.

ASIA. -

Parmotrema sulphuratum (Nees \& Flot.) Hale, Phytologia 28: 339 (1974).

Thailand. Loei (Na Haew N.P.), Nakhon Sri Thammarat ( Khao Luang). Pooprang et al. (1999: 124).

SPECIMENS. BM, RAMK.

AsIA. Indonesia, Malaysia, Papua New Guinea.

Parmotrema thailandicum Elix \& Pooprang, in Pooprang et al., Mycotaxon 72: 117 (1999).

Thailand. Phitsanulok (Phu Hin Rongkla N.P.).

SPECIMENS. CANB (isotype), RAMK (holotype).

ASIA. -

Parmotrema tinctorum (Nyl.) Hale, Phytologia 28: 339 (1974).

Parmelia tinctorum Nyl.

Parmelia coralloidea (Meyen \& Flot.) Vain.

Thailand. Chumphon (Koh Tao I.), Chiang Mai (Doi Suthep N.P., Doi Inthanon N.P., Doi Pacho, Chiang Dao N.P., Queen Sirikit Botanical Garden, Maetang), Chiang Rai, Loei (Ban Sui, Pha Nok Kao), Phitsanulok (Lan Hin Taek, Phu Hin Rongkla, N.P.), UdonThani. Vainio (1921: 37, as Parmelia coralloidea), Sato (1962: 4, as P. tinctorum), Mongkonsuk et al. (1996: 17), Wolseley \& AguirreHudson (1997a: 334; 1997b: 355), Moon et al. (2000a: 104).

SPECIMENS. BKF, BM, CMU, RAMK, TNS, TUR-V, Queen Sirikit Botanical Garden Herbarium.

ASIA. China (incl. Hong Kong), Indonesia, India, Laos, Malaysia, Papua New Guinea, Philippines, Singapore, Sri Lanka, Taiwan.

Parmotrema zollingeri (Hepp) Hale, Phytologia 28: 339 (1974).

ThaILAND. Chiang Mai (Queen Sirikit Botanical Garden), Loei (Ban Su). Moon et al. (2000a: 105).

SPECIMENS. TNS.

AsIA. China, India, Indonesia, Philippines, Taiwan.

Pertusaria DC.; Pertusariaceae

Pertusaria asiana Vain., Suom. Elain-ja Kasvit. Seuran Van. Julk. 1(3): 44 (1921).

ThaIland. Chiang Mai (Doi Suthep N.P.). Vainio (1921: 44); Oshio (1976: 116).

SPECIMEN. TUR-V (holotype).

AsIA. -

Pertusaria bengalensis Vain., Hedwigia 46: 169 (1907).

Thailand. Trat (Koh Chang I.). Vainio (1907: 169; 1909: 110), Oshio (1976: 116).

SPECIMEN. TUR-V (holotype).

AsIA. -
Pertusaria pustulata (Ach.) Duby, Bot. Gall. 2(2): 673 (1830).

THAILAND. Trat (Koh Chang I.). Vainio (1909: 109), Archer (1997: 135).

SPECIMENS. TUR-V.

AsIA. China, India.

Pertusaria sphaerulifera Vain., Hedwigia 46: 169 (1907).

Thailand. Trat (Koh Chang I.). Vainio (1907: 169; 1909: 109).

SPECIMEN. TUR-V (holotype).

ASIA. -

Pertusaria subnegans Vain., Hedwigia 46: 170 (1907).

Thailand. Trat (Koh Chang I.). Vainio (1907: 170; 1909: 110).

SPECIMEN. TUR-V (holotype).

AsIA. -

Phaeographina Müll. Arg.; Graphidaceae

Phaeographina caesiopruinosa (Fée) Müll. Arg., Mém. Soc. Phys. Hist. Nat. Genève 29(8): 49 (1887).

Graphis caesiopruinosa Fée.

THAiland. Chiang Mai (Doi Suthep N.P.). Vainio (1921: 49, as Graphis caesiopruinosa).

SPECIMEN. TUR-V.

ASIA. India, Malaysia, Sri Lanka.

Phaeographina chlorocarpoides (Nyl.) Zahlbr., Cat. Lich. Univ. 2: 321 (1924).

Graphis chlorocarpoides Nyl.

ThaILAND. Chaing Mai (Doi Suthep), Trat (Koh Chang I.). Vainio (1909: 124; 1921: 49, both as Graphis chlorocarpoides), Kashiwadani (1977: 45).

SPECIMENS. TUR-V, TNS.

AsIA. China, Indonesia, Philippines, Taiwan.

Phaeographina chrysenteron (Mont.) Müll. Arg. Hedwigia 30: 52 (1891).

Thailand. Chumphon (Koh Tao I.). Vainio (1921: 49), Paulson (1930: 100).

SPECIMEN. TUR-V, BM.

ASIA. China, India, Indonesia, Sri Lanka.

Phaeographina heterocarpoides (Nyl.) Zahlbr., Cat. Lich. Univ. 2: 439 (1924).

Graphis heterocarpoides Nyl.

Thailand. Trat (Koh Chang I.). Vainio (1909: 123, as Graphis heterocarpoides). 
SPECIMEN. TUR-V.

AsIA. -

Phaeographina persimilis (Vain.) Zahlbr., Cat. Lich. Univ. 2: 442 (1923).

Graphis persimilis Vain.

ThaILAND. Trat (Koh Chang I.). Vainio (1909: 123, as Graphis persimilis).

SPECIMENS. TUR-V.

ASIA. -

Phaeographina scalpturata (Ach.) Müll. Arg., Flora 65: 399 (1882).

Graphis scalpturata Ach.

ThaIlAnd. Chiang Mai (Doi Suthep). Vainio (1921: 49, as Graphis scalpturata).

SPECIMENS. TUR-V.

ASIA. China (Hong Kong), India, Indonesia.

Phaeographina schmidtii (Vain.) Zahlbr., Cat. Lich. Univ.

2: 446 (1923).

Graphis schmidtii Vain.

Thailand. Trat (Koh Chang I.). Vainio (1907: 176; 1909: 124, both as Graphis schmidtii).

SPECIMEN. TUR-V.

ASIA. -

Phaeographina subintricata (Kremp.) Miill. Arg., Bull. Herb. Boissier 3: 320 (1895).

Graphis subintricata Vain.

ThaILAND. Trat (Koh Kong I.). Vainio (1909: 126, as Graphis subintricata).

SPECIMEN. TUR-V.

AsiA. China, India.

Phaeographina subrigida (Nyl.) Zahlbr., Cat. Lich. Univ. 2: 446 (1923).

Graphis subrigida Nyl.

Thailand. Chiang Mai (Doi Suthep). Vainio (1909: 122, as Graphis subrigida).

SPECIMEN. TUR-V.

ASIA. -

Phaeographis Müll. Arg.; Graphidaceae

Phaeographis exaltata (Mont. \& Bosch) Müll. Arg., Flora 65: 336 (1882).

Graphis diversa Nyl.

Thailand. Trat (Koh Chang I.). Vainio (1909: 129, as Graphis diversa).
SPECIMEN. TUR-V.

ASIA. Indonesia, Philippines.

Phaeographis nylanderi (Vain.) Zahlbr., Cat. Lich. Univ. 2: 382 (1923).

Graphis nylanderi Vain.

ThaILAND. Trat (Koh Chang I.). Vainio (1909: 130, as Graphis nylanderi).

SPECIMEN. TUR-V.

ASIA. -

Phaeographis rugulosa (Vain.) Zahlbr., Cat. Lich Univ. 2: 444 (1923).

Graphis rugulosa Vain.

Thalland. Chiang Mai (Doi Suthep). Vainio (1921: 49, as Graphis rugulosa).

SPECIMEN. TUR-V (holotype).

ASIA. -

Phaeographis sericea (Eschw.) Müll. Arg., Flora 71: 523 (1888).

Graphis sericea (Eschw.) Vain.

Thailand. Trat (Koh Chang I.). Vainio (1909: 130, as Graphis sericea).

SPECIMEN. TUR-V.

AsIA. China (Hong Kong).

Phaeographis subtigrina (Vain.) Zahlbr., Cat. Lich. Univ. 2: 387 (1923).

Graphis subtigrina Vain.

ThAILAND. Trat (Koh Chang I.). Vainio (1909: 131, as Graphis subtigrina), Patwardhan \& Kulkarni (1977b: 721), Wirth \& Hale (1978: 29).

SPECIMEN. TUR-V.

ASIA. India.

Phaeophyscia Moberg; Physciaceae

Phaeophyscia hispidula (Ach.) Essl., Mycotaxon 7: 301 (1978).

Physcia setosa (Ach.) Nyl.

Thailand. Chiang Mai (Doi Inthanon N.P.), Chumphon (Koh Tao I.). Vainio (1909: 130), Paulson (1930: 100, as Physcia setosa).

SPECIMENS. BM.

ASIA. China, India, Indonesia, Malaysia.

Phlyctis Wallr.; Phlyctidaceae

[Phlyctis] Phlyctella himalayensis Nyl., Lich. Nov.-Zel.: 73 (1888).

Phlyctis polyphraginia (Nyl.) Vain. 
Thalland. Chiang Mai (Doi Suthep N.P.). Vainio (1921: 43, as Phlyctis polyphragmia).

SPECIMEN. TUR-V.

ASIA. India, Nepal.

[Phlyctis] Phlyctella neozelandica Nyl., Flora 52: 121 (1869).

ThaIland. Chumphon (Koh Tao 1.). Paulson (1930: 100, as Phlyctella neozelandica).

SPECIMEN. BM.

ASIA. -

Phyllopsora Müll. Arg.; Phyllopsoraceae

Phyllopsora furfuracea (Pers.) Zahlbr., in Engler \& Prantl, Nat. Pflanzenfam. 1(1): 138 (1905).

Thalland. Nakhon Ratchasima (Khao Yai N.P.). Boonpragob et al. (1998: 217).

SPECIMENS. BM, RAMK.

AsIA. Papua New Guinea.

Physcia (Schreb.) Michx.; Physciaceae

Physcia sorediosa (Vain.) Lynge, Natur. Skrift. I. Math.Naturvid. Kl. 16: 27 (1924).

ThaIland. Chumphon (Koh Tao I.), Uthai Thani (Huay Kha Khaeng Wildlife Sanctuary). Paulson (1930: 100).

SPECIMEN. BM (not located).

ASIA. Malaysia, Papua New Guinea.

Physcidia Tuck.; Phyllopsoraceae

Physcidia cylindrophora (Taylor) Hue, Bull. Soc. Linn. Normandie 6(1): 97 (1908).

ThaIland. Chiang Mai (Doi Suthep), Nakhon Ratchasima (Khao Yai N.P.). Kalb \& Elix (1995: 286), Boonpragob et al. (1998: 217).

SPECIMENS. BM, RAMK.

AsIA. India, Malaysia, Papua New Guinea, Philippines.

Physcidia wrightii (Tuck.) Tuck., Proc. Amer. Acad. Arts 5: 400 (1862).

ThAILAND. Chiang Mai (Doi Inthanon N.P., Doi Suthep N.P.), Loei (Phu Kradung N.P.), Nakhon Ratchasima (Khao Yai N.P.), Uthai Thani (Huay Kha Khaeng Wildlife Sanctuary). Kurokawa \& Kashiwadani (1988: 15), Kalb \& Elix (1995: 291), Wolseley \& Aguirre-Hudson (1997a: 334), Boonpragob et al. (1998: 217).

SPECIMENS. BM, RAMK, TNS.

ASIA. Malaysia.

Physma A. Massal.; Collemataceae

Physma byrsaeum (Ach.) Tuck., Syn. N. Amer. Lich. 1: 115 (1882).

ThaILAND. Chiang Mai (Doi Suthep N.P.), Uthai Thani (Huay
Kha Khaeng Wildlife Sanctuary). Wolseley \& Aguirre-Hudson (1995: 327).

SPECIMEN. BM.

AsıA. China (Hong Kong), India, Malaysia, Papua New Guinea.

Physma plicatum (Pers.) Hue, Bull. Soc. Linn. Normandie, sér. 5, 9: 136 (1906).

THAILAND. Trat (Koh Chang 1.). Vainio (1909: 116).

SPECIMENS. C, TUR-V.

ASIA, Indonesia.

Platismatia W.L. Culb \& C.F. Culb.; Parmeliaceae

Platismatia erosa W.L. Culb \& C.F. Culb., Contr. U.S. Natl. Herb. 34: 526 (1968).

ThAILAND. Chiang Mai Province (Doi 1nthanon N.P.). Wolseley \& Aguirre-Hudson (1997a: 337).

SPECIMEN. BM.

AsIA. China, 1ndia, Indonesia, Nepal, Philippines, Taiwan, Vietnam.

Polymeridium (Müll. Arg.) R.C. Harris; Trypetheliaceae

Polymeridium albidum (Müll. Arg.) R.C. Harris, Acta Amazonica 14 (Suppl.): 69 (1984) ['1986'].

Arthopyrenia siamensis Vain.

ThaILAND. Chiang Mai (Doi Suthep). Vainio (1921: 55, as Arthopyrenia siamensis), Harris (1993: 624).

SPECIMEN. TUR-V (holotype of Arthopyrenia siamensis).

AsIA. Malaysia, Papua New Guinea, Philippines.

Polymeridium catapastum (Nyl.) R.C. Harris, Acta Amazonica 14 (Suppl.): 70 (1984) ['1986'].

ThAILAND. Nakhon Ratchasima (Khao Yai N.P.). Vongshewarat et al. (1999: 231).

SPECIMENS. CANB, RAMK.

ASIA. Malaysia, Papua New Guinea.

Polymeridium quinqueseptatum (Nyl.) R.C. Harris, in Tucker \& Harris, Bryologist 83: 12 (1980).

ThAILAND. Nakhon Ratchasima (Khao Yai N.P.). Vongshewarat et al. (1999: 231).

SPECIMENS. CANB, RAMK.

AsIA. China (Hong Kong), India, Malaysia, Philippines.

Porina Ach.; Trichotheliaceae

Porina aff. albicera (Kremp.) Overeem, Bull. Jard. Bot. Buitenzorg, sér. 3, 4: 112 (1922).

ThaILAND. Nakhon Ratchasima (Khao Yai N.P.). Boonpragob et al. (1998: 217). 
SPECIMENS. CANB.

AsıA. India, Indonesia, Malaysia, Papua New Guinea, Philippines, Sri Lanka, Vietnam.

Porina americana Fée, Essai Crypt. Écorc. Offic.: 83 (1824).

THAILAND. Nakhon Ratchasima (Khao Yai N.P.). Boonpragob et al. (1998: 217).

SPECIMENS. CANB.

ASIA. -

Porina bellendenica Müll. Arg., Hedwigia 30: 56 (1891).

THAILAND. Nakhon Ratchasima (Khao Yai N.P.). Boonpragob et al. (1998: 217).

SPECIMEN. CANB.

ASIA. -

Porina conica R. Sant., Symb. Bot. Upsal. 12(1): 232 (1952).

Thalland. Nakhon Ratchasima (Khao Yai N.P.). Boonpragob et al. (1998: 217).

SPECIMENS. CANB.

ASIA. India, Indonesia, Malaysia, Papua New Guinea, Philippines, Sri Lanka, Vietnam.

Porina corruscans (Rehm) R. Sant., Symb. Bot. Upsal. 12(1): 223 (1952).

ThAILAND. Nakhon Ratchasima (Khao Yai N.P.). Boonpragob et al. (1998: 217).

SPECIMENS. CANB.

ASIA. India, Indonesia, Papua New Guinea, Philippines.

Porina eminentior (Nyl.) P.M. McCarthy, Lichenologist 32: 42 (2000).

Clathroporina eminentior (Nyl.) Müll. Arg.

ThaILAND. Chiang Mai (Doi Inthanon), Uthai Thani (Khao Nang Rum, Khlong Plou). McCarthy (1995: 329, as Clathroporina eminentior).

SPECIMENS. BM.

AsIA. Papua New Guinea.

Porina epiphylla (Fée) Fée, Essai Crypt. Écorc., Suppl. 1: 76 (1837).

Micropeltis schmidtiana Rostrup.

THAILAND. Nakhon Nayok (Khao Yai N.P.), Nakhon Ratchasima (Khao Yai N.P.), Trat (Koh Chang I.). Rostrup (1902: 211); Santesson (1952: 234), Boonpragob et al. (1998: 217).

\section{SPECIMENS. CANB.}

ASIA. China, India, Indonesia, Malaysia, Papua New Guinea, Philippines, Sri Lanka, Vietnam.

Porina erawanensis P.M. McCarthy \& Vongshewarat, in Vongshewarat et al., Mycotaxon 71: 231 (1999).
THAILAND. Kanchanaburi (Erawan N.P.). Vongshewarat et al. (1999: 231).

SPECIMENS. CANB (holotype), RAMK (isotype).

ASIA. -

Porina internigrans (Nyl.) Müll. Arg., Rep. Australas. Assoc. Advancem. Sci. 1895: 452 (1895).

THAILAND. Sukothai (Ramkhamhaeng N.P.). Vongshewarat et al. (1999: 233).

SPECIMEN. RAMK.

Ası. India, Papua New Guinea.

Porina kansriae P.M. McCarthy, Lichenologist 31: 239 (1999).

THAILAND. Nakhon Ratchasima (Khao Yai N.P.). Boonpragobetal. (1998: 217, as Porina aff. ulceratula Zahlbr.), McCarthy (1999: 239).

SPECIMENS. CANB (incl. holotype).

ASIA. -

Porina lucida R. Sant., Symb. Bot. Upsal. 12(1): 240 (1952).

THAILAND. Nakhon Ratchasima (Khao Yai N.P.). Boonpragob et al. (1998: 217).

SPECIMENS. CANB.

Asia. Indonesia, Malaysia, Papua New Guinea, Philippines, Sri Lanka.

Porina mastoidea (Ach.) Müll. Arg., Bot. Jahrb. Syst. 6: 399 (1885).

THAILAND. Nakhon Ratchasima (Khao Yai N.P.). Boonpragob et al. (1998: 217).

SPECIMENS. CANB.

ASIA. India, Malaysia, Papua New Guinea, Philippines.

Porina aff. octomera (Müll. Arg.) F. Schill., Hedwigia 67: 274 (1927).

ThAILAND. Nakhon Ratchasima (Khao Yai N.P.). Boonpragob et al. (1998: 217).

SPECIMENS. CANB.

ASIA. Vietnam.

Porina rufula (Kremp.) Vain., Acta Soc. Fauna Fl. Fenn. 7(2): 227 (1890).

ThAILAND. Nakhon Ratchasima (Khao Yai N.P.). Boonpragob et al. (1998: 217).

SPECIMENS. CANB.

ASIA. India, Indonesia, Malaysia, Papua New Guinea, Philippines, Sri Lanka.

Porina siamensis P.M. McCarthy, Lichenologist 31: 242 (1999).

THAILAND. Nakhon Nayok (Khao Yai N.P.), Nakhon Ratchasima (Khao Yai N.P.). McCarthy (1999: 242). 
SPECIMENS. CANB (incl. holotype).

ASIA. -

Porina tetracerae (Ach.) Müll. Arg., Bot. Jahrb. Syst. 6: 401 (1885).

ThaIland. Nakhon Ratchasima (Khao Yai N.P.). Boonpragob et al. (1998: 217).

SPECIMENS. CANB.

ASIA. China, India, Malaysia, Papua New Guinea, Sri Lanka.

Porina aff. vezdae Lücking, Nova Hedwigia 52: 285 (1991).

THAILAND. Nakhon Ratchasima (Khao Yai N.P.). Boonpragob et al. (1998: 212).

SPECIMENS. CANB.

AsIA. -

Porina virescens (Kremp.) Müll. Arg., Flora 66: 331 (1883).

ThAILAND. Nakhon Ratchasima (Khao Yai N.P.). Boonpragob et al. (1998: 212).

SPECIMENS. CANB.

ASIA. India, Indonesia, Malaysia, Papua New Guinea, Philippines, Sri Lanka, Vietnam.

Porina wolseleyae P.M. McCarthy, Lichenologist 31: 244 (1999).

THAIlAND. Nakhon Nayok (Khao Yai N.P.). McCarthy (1999: 244).

SPECIMENS. CANB (holotype).

ASIA. -

\section{Pseudocyphellaria Vain.; Lobariaceae}

Pseudocyphellaria argyracea (Delise) Vain., Hedwigia 37: 35 (1898).

THAILAND. Nakhon Ratchasima (Khao Yai N.P.). Boonpragob et al. (1998: 217).

SPECIMEN. RAMK.

AsIA. China (incl. Hong Kong), India, Indonesia, Malaysia, Papua New Guinea, Philippines, Sri Lanka, Taiwan.

Pseudocyphellaria aurata (Sm.) Vain., Acta Soc. Fauna Fl. Fenn. 7: 183 (1890).

ThaIland. Chiang Mai (Doi Inthanon N.P., Doi Suthep N.P.), Uthai Thani (Huay Kha Khaeng Wildlife Sanctuary). Wolseley \& Aguirre-Hudson (1997a: 334).

SPECIMENS. BKF.

ASIA. China, India, Indonesia, Malaysia, Philippines, Taiwan.

Pseudocyphellaria beccarii (Kremp.) D.J. Galloway, Bull. nat. Hist. Mus. London (Bot.) 24: 119 (1994).
ThaILAND. Chiang Mai (Doi Inthanon N.P.). Wolseley \& AguirreHudson (1997a: 334).

SPECIMEN. BM.

AsIA. Malaysia.

Pseudopyrenula Müll. Arg.; Trypetheliaceae

Pseudopyrenula diluta var. degenerans Vain., Bot. Tidsskr. 29: 148 (1909).

Pseudopyrenula endoxanthoides Vain.

ThaIland. Trat (Koh Chang I.). Vainio (1909: 148), Harris (1998: 138).

SPECIMEN. TUR-V (holotype).

ASIA. -

Pyrenula Ach.; Pyrenulaceae

Pyrenula anomala (Ach.) Vain., Ann. Acad. Sci. Fenn., ser. A, 6(7): 189 (1915).

ThaIland. Buri Ram (Khao Kadong), Chanthaburi (Numtokphliu N.P.), Chiang Mai (Queen Sirikit Botanical Garden), Lumpang (Khuntan), Sakon Nakhon (Phu Pan N.P.). Vongshewarat et al. (1999: 234).

SPECIMENS. RAMK.

ASIA. India, Papua New Guinea, Philippines, Vietnam.

Pyrenula approximata Vain., Bot. Tidsskr. 29: 145 (1909).

ThaIlAnd. Trat (Koh Chang I.). Vainio (1909: 145).

SPECIMEN. TUR-V (holotype).

ASIA. -

Pyrenula aspistea Ach., Ges. Naturf. Freunde Berlin Mag. Neuesten Entdeck. Gesammten Naturk. 6: 17 (1814).

ThaILAND. Trat (Koh Chang I.). Vainio (1909: 147).

SPECIMENS. RAMK, TUR-V.

ASIA. India, Indonesia, Papua New Guinea, Philippines, Sri Lanka.

Pyrenula confinis (Nyl.) R.C. Harris, More Florida Lichens: 109 (1995).

Bottaria confinis (Nyl.) Vain.

ThaIland. Trat (Koh Chang I.). Vainio (1909: 143, as Bottaria confinis).

SPECIMENS. RAMK, TUR-V.

AsIA. India, Papua New Guinea, Philippines.

Pyrenula feracissima Vain., Bot. Tidsskr. 29: 145 (1909).

Thailand. Trat (Koh Chang I.). Vainio (1909: 145).

SPECIMENS. TUR-V (holotype).

ASIA. -

Pyrenula glabriuscula (Nyl.) Vain., Bot. Tidsskr. 29: 146 (1909). 
Thailand. Trat (Koh Chang I.). Vainio (1909: 146).

SPECIMENS. TUR-V (holotype).

ASIA. China, Philippines.

Pyrenula leucostoma Ach., Syn. Meth. Lich.: 124 (1914).

Bottaria libricola (Feé) Vain.

Thailand. Trat (Lem Ngob). Vainio (1909: 142, as Bottaria libricola).

SPECIMENS. TUR-V.

AsIA. Indonesia, Papua New Guinea.

Pyrenula macularis (Zahlbr.) R.C. Harris, Mem. New York Bot. Gard. 49: 94 (1989).

THAiland. Rayong. Vongshewarat et al. (1999: 234).

SPECIMENS. RAMK.

ASIA. Papua New Guinea.

Pyrenula nitida (Weig.) Ach., Ges. Naturf. Freunde Berlin Mag. Neuesten Entdeck. Gesammten Naturk. 6: 21 (1814).

Thailand. Chumphon (Koh Tao I.). Paulson (1930: 100).

SPECIMEN. BM (not located).

ASIA. China, India, Indonesia, Sri Lanka.

Pyrenula parvinuclea (Meyen \& Flot.) Aptroot, in Aptroot et al., Biblioth. Lichenol. 64: 165 (1997).

Bottaria denudata (Nyl.) Vain.

Thailand. Chachesoa, Samut Prakan (Muang Boran), Trat (Koh Chang. I.). Vainio (1909: 143, as Bottaria denudata), Vongshewarat et al. (1999: 234).

SPECIMENS. CANB, RAMK, TUR-V.

ASIA. India, Indonesia, Malaysia, Papua New Guinea.

Pyrenula subaggregata Müll. Arg., Bot. Jahrb. Syst. 6: 410 (1885).

Thailand. Trat (Koh Chang I.). Vainio (1909: 144).

SPECIMEN. TUR-V.

ASIA. India.

Pyrenula subferruginea (Malme) R.C. Harris, Mem. New York Bot. Gard. 49: 101 (1989).

Thailand. Kanchanaburi (Erawan N.P.). Vongshewarat et al. (1999: 235).

SPECIMEN. RAMK.

AsIA. Papua New Guinea.

Pyrenula submarginata Vain., Bot. Tidsskr. 29: 146 (1909).

ThaILAND. Trat (Koh Chang I.). Vainio (1909: 146).

SPECIMEN. TUR-V (holotype).

ASIA. -
Pyrenula velatior Müill. Arg., Flora 68: 20 (1885).

Thailand. Chumphon (Koh Tao I.). Paulson (1930: 100).

SPECIMEN. BM.

ASIA. -

Pyrgillus Nyl.; Pyrenulaceae

Pyrgillus indicus (Kremp.) Aptroot, Biblioth. Lichenol. 44: 84 (1991).

Pyrgillocarpon indicum (Kremp.) Nadv.

ThaILAND. Chiang Mai (Doi Inthanon, N.P., Doi Suthep N.P.), Uthai Thani (Huay Kha Khaeng Wildlife Sanctuary). Wolseley \& Aguirre-Hudson (1997a: 334, 339, as Pyrgillocarpon indicum).

SPECIMENS. BM.

AsIA. India, Malaysia.

Pyrgillus javanicus (Mont. \& Bosch) Nyl., Mém. Soc. Sci. Nat. Cherbourg 5: 334 (1858).

THAILAND. Nakhon Ratchasima (Khao Yai N.P.), Uthai Thani (Huay Kha Khaeng Wildlife Sanctuary). Wolseley \& AguirreHudson (1997a: 334, 339), Boonpragob et al. (1998: 217).

SPECIMENS. BM, RAMK.

ASIA. Indonesia, Malaysia, Papua New Guinea, Philippines.

Pyrrhospora Körb.; Lecanoraceae

Pyrrhospora russula (Ach.) Hafellner, in Kalb \& Hafellner, Herzogia 9: 86 (1992).

Lecidea russula Ach.

THAILAND. Chiang Mai (Doi Suthep N.P., Doi Inthanon N.P., Chiang Dao N.P.), Nakhon Ratchasima (Khao Yai N.P.), Uthai Thani (Huay Kha Khaeng Wildlife Sanctuary). Vainio (1921: 48, as Lecidea russula), Sato (1962: 3, as L. russula), Hafellner (1993: 737), Wolseley \& Aguirre-Hudson (1997a: 333, 339; 1997b: 356), Boonpragob et al. (1998: 217).

SPECIMENS. BM, CMU, TNS, TUR-V.

AsIA. China (Hong Kong).

Pyxine Fr.; Physciaceae

Pyxine coccifera (Fée) Nyl., Mém. Soc. Imp. Sci. Nat. Cherbourg 5: 108 (1857).

ThaIland. Chiang Mai (Doi Suthep N.P., Doi Inthanon N.P.), Lampang (Doi Khun Tan N.P.), Uthai Thani (Huay Kha Khaeng Wildlife Sanctuary). Vainio (1921: 45), Wolseley \& Aguirre-Hudson (1991: 172, 173; 1997a: 334; 1997b: 355).

SPECIMENS. BKF, BM, CMU, RAMK.

ASIA. India, Indonesia, Malaysia, Sri Lanka, Vietnam.

Pyxine consocians Vain., Philipp. J. Sci., Sect. C, 8: 109 (1913).

Thalland. Chiang Mai, Uthai Thani. Wolseley \& Aguirre-Hudson (1997a: 334; 1997b: 355, 356). 
SPECIMENS. BKF, BM, CMU.

ASIA. India, Malaysia, Papua New Guinea, Philippines.

Pyxine coralligera Malme, Bih. Kongl. Svenska VetenskAkad. Handl. 23(3): 40(1897).

ThAILAND. Chiang Mai (Doi Inthanon N.P., Doi Suthep N.P.), Uthai Thani (Huay Kha Khaeng Wildlife Sanctuary). Wolseley \& Aguirre-Hudson (1997a: 334; 1997b: 356).

SPECIMENS. BKF, BM, CMU.

Ası. India, Papua New Guinea.

Pyxine retirugella Nyl., Ann. Sci. Nat. Bot., sér. 4, 11: 240 (1859).

Pyxine asiatica Vain.

Pyxine retirugella var. laevior Vain.

Pyxine copelandii Vain.

ThaILAND. Trat (Koh Chang I.), Uthai Thani (Huay Kha Khaeng Wildlife Sanctuary). Vainio (1909: 113, as Pyxine asiatica, P. retirugella var. laevior and $P$. copelandii). Rogers (1986: 149).

SPECIMENS. BM, TUR-V.

ASIA. India, Malaysia, Papua New Guinea, Philippines.

Pyxine schmidtii Vain., Hedwigia 46: 170 (1907).

Thailand. Trat (Koh Chang I.). Vainio (1909: 112).

SPECIMEN. TUR-V (holotype).

ASIA. -

Ramalina Ach.; Ramalinaceae

Ramalina calicaris (L.) Rohl, Deutshl. Fl. 3(2): 139 (1813).

THaIland. Chiang Mai (Doi Suthep N.P., Chiang Dao N.P.). Sato (1962: 6), Yoshimura (1978: 37).

SPECIMENS. C.

AsIA. China, India, Indonesia, Taiwan.

Ramalina conduplicans Vain., Suom. Elain-ja Kasvit. Seuran Van. Julk. 1(3): 35 (1921).

THAILAND. Chiang Mai (Doi Inthanon N.P., Doi Suthep N.P.). Vainio (1921: 35), Awasthi (1960: 177), Sato (1962: 6).

SPECIMENS. TUR-V (syntypes).

ASIA. -

Ramalina farinacea (L.) Ach., Methodus: 263 (1803).

ThaIland. Chiang Mai (Chiang Dao N.P.), Chumphon (Koh Tao I.), Trat (Koh Chang I.). Vainio (1909: 104), Paulson (1930: 100), Yoshimura (1978: 37).

SPECIMENS. BM, C, TUR-V.

ASIA. China, Indonesia, Sri Lanka, Vietnam.

Ramalina hossei Vain., Suom. Elain-ja Kasvit. Seuran Van. Julk. 1(3): 36 (1921).
Thalland. Chiang Mai (Doi Suthep N.P.). Vainio (1921: 36), Kashiwadani (1988: 129-133).

SPECIMENS. TNS, TUR-V (holotype).

ASIA. Taiwan.

Relicina (Hale \& Kurok.) Hale; Parmeliaceae

Relicina abstrusa (Vain.) Hale, Phytologia 28: 484 (1974).

ThaILAND. Nakhon Ratchasima (Khao Yai N.P), Loei (Phu Kradung N.P.), Uthai Thani (Huay Kha Khaeng Wildlife Sanctuary). Culberson et al. (1981: 25), Boonpragob et al. (1999: 218), Moon et al. (2000a: 105).

SPECIMENS. BM, RAMK, TNS.

ASIA. China, India, Indonesia, Malaysia, Philippines, Taiwan.

Relicina circumnodata (Nyl.) Hale, Phytologia 28: 484 (1974).

ThaIland. Phitsanulok (Lan Hin Taek, Phu Hin Rongkla, N.P.), Phuket (Ton Sai). Moon et al. (2000a: 105).

SPECIMENS. TNS.

AsIA. Malaysia, Philippines.

Relicina subabstrusa (Gyeln.) Hale, Phytologia 28: 485 (1974).

ThaIland. Nakhon Ratchasima (Khao Yai N.P). Boonpragob et al. (1999: 218).

SPECIMENS. RAMK.

ASIA. Malaysia, Philippines, Taiwan.

Relicina sublanea (Kurok.) Hale, Phytologia 28: 485 (1975).

Thailand. Yala (Wang Pra, Bang-Lang N.P.). Elix (1996: 120). SPECIMENS. PSU.

ASıA. Indonesia, Malaysia, Philippines, Sri Lanka.

Relicina sublimbata (Nyl.) Hale, Phytologia 28: 485 (1974).

ThaIland. Loei (Phu Kradung). Hale (1975: 31), Wolseley \& Aguirre-Hudson (1995: 328), Moon et al. (2000a: 103).

SPECIMENS. BM, TNS, US.

AsIA. Myanmar.

Relicinopsis Elix \& Verdon; Parmeliaceae

Relicinopsis intertexta (Mont. \& Bosch) Elix \& Verdon, Mycotaxon 27: 281 (1986).

Parmelia ecoronata Nyl.

Thailand. Trat (Koh Chang I.). Vainio (1909: 107, as Parmelia ecoronata).

SPECIMENS. BM, TUR-V.

AsIA. India, Indonesia, Philippines, Malaysia, Papua New Guinea. 
Relicinopsis rahengensis (Vain.) Elix \& Verdon, Mycotaxon 27: 282 (1986).

Parmelia rahengensis Vain. Pseudoparmelia rahengensis (Vain.) Hale.

Thailand. Nong Boa near Raheng, Chiang Mai (Doi Saget, Doi Suthep N.P., Doi Inthanon N.P., Chieng Dao N.P., Queen Sirikit Botanical Garden, Maetang), Lampang (Doi Khun Tan N.P.), Phitsanulok (Lan Hin Taek, Phu Hin Rongkla, N.P.), Uthai Thani (Huay Kha Khaeng Wildlife Sanctuary). Vainio (1921: 39, as Parmelia rahengensis), Hale (1976a: 42, as Pseudoparmelia rahengensis), Wolseley \& Aguirre-Hudson (1995: 329; 1997a: 334; 1997b: 335, 366), Moon et al. (2000a: 105).

SPECIMENS. BKF, BM, CMU, TNS, US, TUR-V (lectotype).

ASIA. -

Relicinopsis malaccensis (Nyl.) Elix \& Verdon, Mycotaxon 27: 282 (1986).

Thailand. Loei (Nahaew N.P.), Phitsanulok (Phu Hin Rongkla N.P.). Pooprang et al. (1999: 125).

SPECIMENS. RAMK.

ASIA. India, Indonesia, Malaysia, Philippines, Sri Lanka.

Rimelia Hale \& A. Fletcher; Parmeliaceae

Rimelia reticulata (Taylor) Hale \& A. Fletcher, Bryologist 93: 28 (1990).

Parmelia cetrata f. soredifera Vain.

Rimelia clavulifera (Räsänen) Kurok.

Thailand. Chiang Mai (Doi Suthep N.P., Doi Inthanon N.P., Maetang), Chiang Rai (Ban Lang), Loei (Phu Kradung), Phitsanulok (Lan Hin Taek, Phu Hin Rongkla, N.P.). Vainio (1921: 37, as Parmelia cetrata f. soredifera), Sato (1962: 3), Wolseley \& Aguirre-Hudson (1995: 329), Moon et al. (2000a: 105, as Rimelia clavulifera).

SPECIMENS. BKF, BM, CMU, TNS, TUR-V.

Asıa. China, India, Indonesia, Malaysia, Papua New Guinea, Sri Lanka, Taiwan.

Roccella DC.; Roccellaceae

Roccella phycopsis Ach., Lichenogr. Universalis: 440 (1803).

Thailand. Chumphon (Koh Tao I.). Paulson (1930: 100).

SPECIMENS. BM.

ASIA. -

Sarcographa Fée; Graphidaceae

Sarcographa labyrinthica Müll. Arg., Mém. Soc. Phys. Hist. Nat. Genève 29(8): 46 (1887).

Graphis labyrinthica (Ach.) Vain.

Thalland. Trat (Koh Chang I.). Vainio (1909: 131, as Graphis labyrinthica).

SPECIMENS. BM, TUR-V.
ASIA. Indonesia, Malaysia, Sri Lanka.

Sarcographa medusulina (Nyl.) Müll. Arg., Flora 70: 77 (1887).

Thailand. Chiang Mai (Doi Suthep N.P.), Nakhon Ratchasima (Khao Yai N.P.), Uthai Thani (Huay Kha Khaeng Wildlife Sanctuary). Vainio (1909: 131), Wolseley \& Aguirre-Hudson (1997a: 334; 1997b: 355), Boonpragob et al. (1998: 218).

SPECIMENS. BM, RAMK.

ASIA. -

Spilonema Bornet; Coccocarpiaceae

Spilonema schmidtii (Vain.) Henssen, Symb. Bot. Upsal. 18: 97 (1963).

Leptogidium mooreii (Hepp) Nyl.

Leptodendriscum schmidtii Vain.

ThAILAND. Trat (Koh Chang I). Vainio (1909: 115; 1921: 34, as Leptodendriscum schmidtii), Henssen (1963: 97).

SPECIMENS. TUR-V (lectotype, syntype).

ASIA. -

Sporopodium Mont.; Ectolechiaceae

Sporopodium hossei Vain., Suom. Elain-ja Kasvit. Seuran Van. Julk. 1(3): 47 (1921).

ThaILAND. Chiang Mai (Doi Suthep N.P.). Vainio (1921: 47).

SPECIMEN. TUR-V (holotype).

ASIA. -

Sporopodium leucoxanthum (Spreng.) Vain., Suom. Elainja Kasvit. Seuran Van. Julk. 1(3): 47 (1921).

Thailand. Chiang Mai (Doi Suthep N.P.). Vainio (1921: 47).

SPECIMEN. TUR-V (holotype).

ASIA. -

Sticta (Schreb.) Ach.; Lobariaceae

Sticta boschiana Mont. \& Bosch., in Junghuhn, $P l$. Junghuhn. 4: 436 (1855).

THAILAND. Chiang Mai (Doi Inthanon N.P., Doi Suthep N.P.). Wolseley (1991: 233).

SPECIMENS. BM.

ASIA. Indonesia, Philippines.

Sticta fuliginosa (Dicks.) Ach., Methodus: 280 (1803).

ThaILAND. Chiang Mai (Doi Inthanon N.P., Doi Suthep N.P.). Wolseley (1991: 233), Wolseley \& Aguirre-Hudson (1997a: 334).

SPECIMENS. BM.

AsIA. China, India, Indonesia, Philippines.

Sticta nylanderiana Zahlbr., Cat. Lich. Univ. 3: 356 (1925). 
ThaILAND. Chiang Mai (Chiang Dao N.P., Doi Inthanon N.P., Doi Suthep N.P., Doi Saget). Yoshimura (1978: 38), Wolseley (1991: 233), Wolseley \& Aguirre-Hudson (1997a: 334).

SPECIMENS. AAU, BM, C.

AsIA. China, India, Taiwan.

Sticta platyphylloides Nyl., in Hue, Bull. Soc. Bot. France 34: 22 (1887).

ThaIland. Chiang Mai (Doi Suthep N.P.). Yoshimura (1978: 38). SPECIMEN. AAU.

ASIA. China, India.

Sticta weigelii Isert, in Acharius, Lichenogr. Universalis: 446 (1810).

THAILAND. Nakhon Nayok (Khao Yai N.P.). Yoshimura (1978: 39).

SPECIMEN. AAU.

AsIA. China (incl. Hong Kong), India, Indonesia.

Sticta wrightii Tuck., Amer. J. Arts, ser. 2, 28: 204 (1859).

ThaIland. Chiang Mai (Doi Suthep N.P.). Vainio (1921: 45).

SPECIMENS. TUR-V.

AsIA. China, Taiwan.

Strigula Fr.; Strigulaceae

Strigula maculata (Cooke \& Massee) R. Sant., Symb. Bot. Upsal. 12(1): 186 (1952).

ThaILAND. Nakhon Ratchasima (Khao Yai N.P.). Boonpragob et al. (1998: 218).

SPECIMENS. CANB.

ASIA. India, Indonesia, Philippines, Sri Lanka, Vietnam.

Strigula melanobapha (Kremp.) R. Sant., Symb. Bot. Upsal. 12(1): 188 (1952).

ThAILAND. Nakhon Ratchasima (Khao Yai N.P.). Boonpragob et al. (1998: 218).

SPECIMENS. CANB.

AsıA. China, India, Malaysia, Papua New Guinea, Philippines, Sri Lanka.

Strigula smaragdula Fr., Linnaea 5: 550 (1830).

THAILAND. Nakhon Ratchasima (Khao Yai N.P.). Boonpragob et al. (1998: 218).

SPECIMENS. CANB.

AsıA. China, India, Indonesia, Malaysia, Nepal, Papua New Guinea, Philippines, Sri Lanka, Taiwan, Vietnam.

Strigula subtilissima (Fée) Müll. Arg., Flora 66: 346 (1863).

ThaILAND. Nakhon Ratchasima (Khao Yai N.P.). Boonpragob et al. (1998: 218).
SPECIMENS. CANB.

AsIA. China, Indonesia, Papua New Guinea, Philippines, Sri Lanka, Taiwan, Vietnam.

Syncesia Taylor; Roccellaceae

Syncesia albiseda (Nyl.) Tehler, Fl. Neotropica 78: 37 (1996).

Thailand. Chiang Mai (Doi Suthep N.P.), Uthai Thani (Huay Kha Khaeng Wildlife Sanctuary). Wolseley \& Aguirre-Hudson (1997a: 339).

SPECIMENS. BM.

ASIA. Sri Lanka.

Teloschistes Norman; Teloschistaceae

Teloschistes flavicans (Sw.) Norman, Nytt Mag. Naturvidensk. 7: 229 (1853).

Teloschistes flavicans f. glaber Vain.

ThaILAND. Trat (Koh Chang I.). Vainio (1909: 111, as Teloschistes flavicans f. glaber).

SPECIMENS. TUR-V.

AsIA. China, India, Indonesia, Sri Lanka.

Thelotrema Ach.; Thelotremataceae

Thelotrema alboan nuliforme Nagarkar, Sethy \& Patw., Kavaka 12(2): 57 (1985).

Thalland. Loei (Na Haeo N.P.), Nakhon Ratchasima (Khao Yai N.P.). Boonpragob et al. (1998: 218), Homchantara (1999: 64-66).

SPECIMENS. RAMK.

ASIA. India.

Thelotrema albosorellum (Nyl.) Tuck., Gen. Lich.: 139 (1872).

THAILAND. Nakhon Ratchasima (Khao Yai N.P.). Boonpragob et al. (1998: 218), Homchantara (1999: 66-67).

SPECIMENS. RAMK.

ASIA. India, Malaysia, Sri Lanka.

Thelotrema astroideum (Berk. \& Broome) Hale, Mycotaxon 11: 131 (1980).

Thailand. Chantaburi (Namtok Phliu N.P.), Nakhon Ratchasima (Khao Yai N.P.). Boonpragob et al. (1998: 218), Homchantara (1999: 69-71).

SPECIMENS. RAMK.

AsIA. India, Malaysia, Sri Lanka.

Thelotrema calathiforme Vain., Hedwigia 46: 174 (1907).

ThaILAND. Trat (Koh Chang I.). Vainio (1909: 119).

SPECIMENS. FH, TUR-V (both syntypes).

AsIA. India. 
Thelotrema canarense Patw. \& C.R. Kulk., Norweg. J. Bot. 24: 128 (1977).

THAILAND. Chiang Mai ( Doi Suthep N.P.), Kanchanaburi (Erawan N.P.), Sakhon Nakhon (Phu Phan N.P.). Homchantara (1999: 7173).

SPECIMENS. BM, RAMK.

AsIA. India.

Thelotrema colobicum Nyl., Bull. Soc. Linn. Normandie, sér. 2, 7: 169 (1873).

THAILAND. Chantaburi (Kitchakut N.P.), Chiang Mai (Doi Suthep N.P.), Loei ( Na Haeo N.P., Phu Ruea N.P.), Nakhon Ratchasima (Khao Yai N.P.), Phetchabun (Phu Hin Rongkla N.P.), Sukhothai (Ramkhamhaeng N.P.), Uthai Thani (Huay Kha Khaeng Wildlife Sanctuary). Boonpragob et al. (1998: 218), Homchantara (1999: 73-76)

SPECIMENS. BM, RAMK.

ASIA. India, Sri Lanka.

Thelotrema dilatatum (Müll. Arg.) Hale, Mycotaxon 11: 13 (1980).

THAILAND. Chantaburi (Namtok Phliu N.P.). Homchantara (1999: 76-78)

SPECIMENS. RAMK.

ASIA. India, Malaysia, Sri Lanka.

Thelotrema expallescens Nyl., Flora 59: 560 (1876).

THAILAND. Nakhon Ratchasima (Khao Yai N.P.). Boonpragob et al. (1998: 218).

SPECIMENS. RAMK.

ASIA. -

Thelotrema glaucopallens Nyl., Ann. Sci. Nat. Bot., sér. 4, 2: 327 (1863).

ThaIland. Chantaburi (Khao Soi Dao N.P., Kitchakut N.P.). Homchantara (1999: 78-81).

SPECIMENS. RAMK.

ASIA. India, Malaysia.

Thelotrema imperfectum Hale, Bull. Brit. Mus. (Nat. Hist.), Bot. 8(3): 255 (1981).

ThaILAND. Uthai Thani (Huay Kha Khaeng Wildlife Sanctuary). Homchantara (1999: 81-83).

SPECIMENS. BM.

ASIA. Sri Lanka.

Thelotrema laceratulum Müll. Arg., Flora 70: 399 (1887).

THAILAND. Lampang (Wiang Kosai N.P.), Nakhon Ratchasima (Khao Yai N.P.), Uthai Thani (Huay Kha Khaeng Wildlife Sanctuary). Homchantara (1999: 84-86).

SPECIMENS. BM, RAMK.

AsIA. India.
Thelotrema lacteum Kremp., in Nylander, Flora 47: 269 (1864).

THAILAND. Sakonnakhon (Phu Pham N.P.). Homchantara (1999: 86-89).

SPECIMENS. RAMK.

ASIA. India, Papua New Guinea, Sri Lanka.

Thelotrema laemense Homchantara \& Coppins,

Lichenologist 34: (in press).

THAILAND. Kanchanaburi (Khao Laem N.P.). Homchantara (1999: 134-136, as Thelotrema 'unidentified 3').

SPECIMENS. E (isotype), RAMK (holotype).

AsIA. -

Thelotrema lepademersum Nagarkar, Sethy \& Patw., Kavaka 12(2): 59 (1985).

ThaILAND. Chantaburi (Namtok Phliu N.P.), Nakhon Ratchasima (Khao Yai N.P.). Homchantara (1999: 89-91).

SPECIMENS. RAMK.

AsIA. India.

Thelotrema leprieurii (Mont.) Hale, Mycotaxon 11: 131 (1980).

ThaILAND. Chantaburi (Namtok Phliu N.P.). Boonpragob et al. (1998: 218), Homchantara (1999: 91-93).

SPECIMENS. RAMK.

AsIA. India, Indonesia, Sri Lanka.

Thelotrema leprocarpoides Hale, Bull. Brit. Mus. (Nat. Hist.), Bot. 8(3): 259 (1981).

THAILAND. Nakhon Ratchasima (Khao Yai N.P.). Boonpragob et al. (1998: 218).

SPECIMENS. RAMK.

ASIA. India.

Thelotrema leprocarpum (Nyl.) Tuck., Gen. Lich.: 139 (1872).

Thailand. Loei (Na Haeo N.P.), Sukhothai (Ramkhamhaeng N.P.). Homchantara (1999: 94-96).

SPECIMENS. RAMK.

ASIA. India, Indonesia, Sri Lanka.

Thelotrema monosporum Nyl., Ann. Sci. Nat. Bot., sér. 4, 15: 46 (1860).

Thalland. Chiang Mai (Doi Suthep N.P.), Uthai Thani (Huay Kha Khaeng Wildlife Sanctuary). Homchantara (1999: 96-100).

SPECIMENS. BM.

ASIA. India, Sri Lanka.

Thelotrema mongkolsukii Homchantara \& Coppins, Lichenologist 34: (in press).

THAILAND. Sukhothai (Ramkhamhaeng N.P.). Homchantara (1999: 129-131, as Thelotrema 'unidentified 1'). 
SPECIMENS. E (isotype), RAMK (holotype).

ASIA. -

Thelotrema nureliyum Hale, Bull. Brit. Mus. (Nat. Hist.), Bot. 8(3): 259 (1981).

ThAILAND. Nakhon Ratchasima (Khao Yai N.P.). Boonpragob et al. (1998: 218), Homchantara (1999: 100-102).

SPECIMENS. RAMK.

ASIA. Sri Lanka.

Thelotrema patens Nyl., Acta. Soc. Sci. Fenn. 26: 17 (1900).

Thailand. Chiang Mai (Doi Suthep), Phetchabaun (Phu Hin Rongkla N.P.). Homchantara (1999: 102-104).

SPECIMENS. BM, RAMK.

ASıA. Malaysia, Sri Lanka.

Thelotrema phliuense Homchantara \& Coppins, Lichenologist 34: (in press).

THAILAND. Chantaburi (Namtok Phliu N.P.). Homchantara (1999: 138-140, as Thelotrema 'unidentified 5').

SPECIMENS. BM (holotype).

ASIA. -

Thelotrema phlyctidioides (Müll. Arg.) Hale, Mycotaxon 11: 132 (1980).

Thalland. Chantaburi (Namtok Phliu N.P.), Chiang Mai ( Doi SuthepN.P.), Nakhon Ratchasima (Khao Yai N.P.), Uthai Thani(Huay Kha Khaeng Wildlife Sanctuary). Homchantara (1999: 104-117).

SPECIMENS. BM, RAMK.

ASIA. Sri Lanka.

Thelotrema piluliferum Tuck., Proc. Amer. Acad. Arts 7: 227 (1868).

ThaIland. Chiang Mai (Doi Suthep N.P.). Homchantara: 1999: 107-108.

SPECIMENS. BM.

ASıA. Malaysia, Sri Lanka.

Thelotrema platysporum Harm., Bull. Séances Soc. Sci. Nancy, sér. 3, 13: 41 (1912).

ThAIlAND. Nakhon Ratchasima (Khao Yai N.P.). Homchantara (1999: 110-112).

SPECIMENS. BM, RAMK.

ASIA. India, Malaysia, Papua New Guinea, Sri Lanka.

Thelotrema poeltii Patw. \& C.R. Kulk., Norweg. J. Bot. 24: 130 (1977).

Thailand. Nakhon Ratchasima (Khao Yai N.P.). Boonpragob et al. (1998: 218).

SPECIMENS. RAMK.

ASIA. India.
Thelotrema porinoides Mont. \& Bosch, in Junghuhn, Enum. Pl. Insul. Java Sumatra: 151 (1855).

ThaIland. Nakhon Ratchasima (Khao Yai N.P.). Boonpragob et al. (1998: 218), Homchantara (1999: 114-117).

SPECIMENS. RAMK.

ASIA. India, Indonesia, Malaysia, Papua New Guinea, Philippines.

Thelotrema pseudoexanthismocarpum (Patw. \& C.R. Kulk.) Hale, Mycotaxon 11: 132 (1980).

Thailand. Chiang Mai (Doi Suthep N.P.), Satun (Thalae Ban N.P.), Sukhothai (Ramkhamhaeng N.P.), Uthai Thani (Huay Kha Khaeng Wildlife Sanctuary). Homchantara (1999: 117-120).

SPECIMENS. RAMK.

AsIA. India.

Thelotrema pycnophragmium Nyl., Sert. Lich. Trop. Labuan Singapore: 5 (1891).

ThaILAND. Chantaburi (Namtok Phliu N.P.). Homchantara (1999: 120-122).

SPECIMENS. RAMK.

AsıA. India, Malaysia.

Thelotrema rhododiscum Homchantara \& Coppins, Lichenologist 34: (in press).

Thailand. Nakhon Ratchasima (Khao Yai N.P.). Homchantara (1999: 136-138, as Thelotrema 'unidentified 4').

SPECIMENS. E (isotype), RAMK (holotype).

ASIA. -

Thelotrema rugatulum Nyl., Bull. Soc. Linn. Normandie, sér. 2, 7: 168 (1873).

Thalland. Nakhon Ratchasima (Khao Yai N.P.). Boonpragob et al. (1998: 218).

SPECIMENS. RAMK.

ASIA. India

Thelotrema subhiatum Patw. \& Nagarkar, Biovigyanam 6: 9 (1980).

ThaIland. Chantaburi (Kitchakut N.P.), Phetchabun (Phu Hin Rongkla N.P.), Sakonnakhon (Phu Phan N.P.). Homchantara (1999: 120-122).

SPECIMENS. RAMK.

ASIA. India.

Thelotrema turgidulum (Muill. Arg.) Hale, Mycotaxon 11: 132 (1980).

THAILAND. Nakhon Ratchasima (Khao Yai N.P.). Boonpragob et al. (1998: 218), Homchantara (1999: 125-129).

SPECIMENS, RAMK.

AsIA. India. 
Thelotrema weberi Hale, Phytologia. 27: 497 (1974).

ThaILAND. Chiang Mai ( Doi Suthep N.P.). Homchantara (1999: 107-108).

SPECIMENS. BM.

ASIA. Malaysia, Papua New Guinea, Sri Lanka.

Tricharia Fée emend. R. Sant.; Gomphillaceae.

Tricharia aff. vainioi R. Sant., Symb. Bot. Upsal. 12(1): 382 (1952).

THAILAND. Nakhon Ratchasima (Khao Yai N.P.). Boonpragob et al. (1998: 218).

SPECIMEN. RAMK.

Ası. China, Indonesia, Papua New Guinea, Philippines.

Trypethelium Spreng.; Trypetheliaceae

Trypethelium eluteriae Spreng., Anleit. Kenntn. Gew. 3: 351 (1804).

Thalland. Chumphon (Koh Tao I.), Nakhon Ratchasima (Khao Yai N.P.). Paulson (1930: 100), Boonpragob et al. (1998: 218).

SPECIMENS. BM, CANB, RAMK.

ASIA. China (incl. Hong Kong), India, Indonesia, Malaysia, Papua New Guinea, Taiwan, Vietnam.

Trypethelium nigroporum Makhija \& Patw., J. Hattori Bot. Lab. 73: 202 (1993).

Thalland. Loei (Naheaw), Sakonnakhon (Phu Pan N.P.). Vongshewarat et al. (1999: 235).

SPECIMENS. RAMK.

AsIA. India.

Trypethelium tropicum (Ach.) Müll. Arg., Bot. Jahrb. Syst. 6: 393 (1885).

THAILAND. Nakhon Ratchasima (Khao Yai N.P.). Boonpragob et al. (1998: 218).

SPECIMENS. CANB, RAMK.

AsIA. China (Hong Kong), India, Indonesia, Malaysia, Papua New Guinea, Singapore, Vietnam.

Trypethelium variolosum (Ach.) Müll. Arg., Flora 68: 255 (1885).

Pseudopyrenula ochroleuca (Eschw.) Vain.

Pseudopyrenula ochroleuca var. subdissocians Vain.

Trypethelium ochroleucum var. subdissocians (Vain.) Zahlbr.

Thalland. Chiang Mai (Doi Suthep N.P.), Trat (Koh Chang I.). Vainio (1909: 147; 1921: 55, as Pseudopyrenula ochracea), Harris (1998: 145).

SPECIMEN. TUR-V (holotype of Pseudopyrenula ochroleuca var. subdissocians).

AsIA. India, Indonesia, Malaysia, Papua New Guinea.
Tylophoron Nyl. ex Stizenb.; Caliciales

Tylophoron moderatum Nyl., Bot. Zeitung 20: 279 (1862).

Thalland. Chiang Mai (Doi Suthep N.P.), Uthai Thani (Huay Kha Khaeng Wildlife Sanctuary). Wolseley \& Aguirre-Hudson (1997a: 334, 339).

SPECIMENS. BM.

AsIA. China, India, Taiwan.

Usnea Dill. ex Adans.; Usneaceae

Usnea australis Fr., Syst. Orb. Veg.: 282 (1825).

Thalland. Chiang Mai (Doi Suthep N.P., Doi Inthanon N.P.). Vainio (1921: 33), Sato (1962: 4), Santisuk (1988: 99).

SPECIMEN. TUR-V.

AsIA. -

Usnea baileyi (Stirt.) Zahlbr., Denkschr. Kaiserl. Akad. Wiss., Wien Math.-Naturwiss. Kl. 83: 182, 191 (1909).

Eumitria perrubescens Vain.

Usnea implicita (Stirt.) Zahlbr.

Usnea implicita subsp. perrubescens Stirt.

Thalland. Loei (Phu Luang, Chiang Mai (Doi Suthep N.P.). Vainio (1921: 35, as Eumitria perrubescens), Motyka (1936: 62, as Usnea implicita subsp. perrubescens), Rogers \& Stevens (1988: 358), Yoshimura (1978: 35).

SPECIMENS. AAU, C, TUR-V (holotype of Eumitria perrubescens).

AsIA. China (incl. Hong Kong), Indonesia, Malaysia, Myanmar, Papua New Guinea, Philippines, Taiwan.

Usnea baileyi subsp. chrysopora (Stein) Asahina, J. Jap. Bot. 42 : 7 (1967).

Thalland. Chiang Mai (Doi Suthep N.P., Chiang Dao N.P.). Yoshimura (1978: 36).

SPECIMENS. C.

ASIA. Philippines.

Usnea baileyi subsp. septentrionalis Asahina, in Hara, $F l$. Eastern Himalaya: 598 (1966).

Thailand. Chiang Mai (Doi Suthep N.P.), Loei (Thung Salaeng, Phu Luang N.P.). Yoshimura (1978: 35).

SPECIMENS. AAU, C.

AsIA. India, Nepal, Taiwan.

Usuea dasycera (Nyl.) Motyka, Usnea: 552 (1936-38).

Usnea perplexans f. dasycera (Nyl.) Vain.

THAILAND. Chiang Mai (Doi Suthep N.P.). Vainio (1921: 33, as Usnea perplexans f. dasycera).

SPECIMENS. AAU, C.

ASIA. -

Usuea endochroa var. papillata (Vain.) Zahlbr., Cat. Lich. Univ. 6: 564 (1930).

Eumitria endochroa var. papillata Vain. 
ThaIland. Chiang Mai (Doi Suthep N.P.). Vainio (1921: 34, as Eumitria endochroa var. papillata).

SPECIMEN. TUR-V.

ASIA. Philippines.

Usnea endorhodina (Vain.) Zahlbr., Cat. Lich. Univ. 6: 564 (1930).

Eumitria endorhodina Vain.

ThaIland. Chiang Mai (Doi Suthep N.P.). Vainio (1921: 34, as Eumitria endorhodina).

SPECIMEN. TUR-V.

AsIA. Philippines.

Usnea florida var. subcomosa Vain., Philipp. J. Sci. Sect. C, 4: 652 (1909).

ThaIland. Chiang Mai (Doi Suthep N.P.). Vainio (1921: 33).

SPECIMEN. TUR-V.

AsIA. China, Philippines.

Usnea himantodes Stirt., Scott. Naturalist (Perth) 7: 75 (1883).

Usnea neoguineensis var. gracilior Asahina.

Usnea neoguineensis var. neoguineensis Asahina.

THAILAND. Loei (Phu Luang). Asahina (1968: 497, as Usnea neoguineensis var. gracilior), Yoshimura(1978: 36, as U. neoguineensis and U. neoguineensis var. gracilior), Stevens (1990: 410).

SPECIMENS. AAU, TNS (holotypes of Usnea neoguineensis var. gracilior and Usnea neoguineensis var. neoguineensis).

AsIA. China, Indonesia, Papua New Guinea, Philippines, Taiwan.

Usnea hossei Vain., Suom. Elain-ja Kasvit. Seuran Van.

Julk. 1(3): 34 (1921).

ThaIlAnd. Chiang Mai (Doi Suthep N.P., Doi Inthanon N.P.). Vainio (1921: 34), Zahlbruckner (1933: 581), Sato (1938: 4; 1962: 4), Herre (1963: 65), Santisuk (1988: 99).

SPECIMENS. BKF, TNS, TUR-V (syntypes).

AsIA. China, Malaysia, Taiwan.

Usnea leucospilodea Nyl., J. Linn. Soc. London (Bot.) 20:

30 (1883).

ThaIlAnd. Chiang Mai (Doi Suthep N.P.). Asahina, (1969: 37), Yoshimura (1978: 36).

SPECIMEN. AAU, C.

ASIA. India, Papua New Guinea, Laos, Malaysia, Taiwan.

Usnea luridorufa Stirt., Scott. Naturalist (Perth) 7: 104 (1881).

ThaILAND. Doi Suthep. Motyka (1937-38: 523), Awasthi (1986: 374).

SPECIMEN. AAU, C.

ASIA. China.
Usnea pectinata Taylor, in Hooker, J. Bot. (London) 6: 191 (1847).

ThaIland. Chiang Mai (Doi Suthep N.P.), Loei (Phu Kradung N.P., Phu Luang N.P.). Awasthi (1986: 362), Yoshimura (1978: 36), Kurokawa \& Kashiwadani (1988: 51).

SPECIMENS. Kurokawa, Lich. Rar. Crit. Exs. No. 347, C, AAU.

AsIA. China, India, Malaysia.

Usnea perplexans Stirt. Scott. Naturalist (Perth) 6: 103 (1881).

Thalland. Chiang Mai (Doi Suthep N.P.). Vainio (1921: 33).

SPECIMEN, AAU.

ASIA. India, Philippines.

Usnea rubicunda Stirt., Scott. Naturaliust (Perth) 6: 102 (1881).

Thailand. Chiang Mai (Doi Suthep N.P), Phu Miang. Yoshimura (1978: 36).

SPECIMENS. AAU.

AsIA. China, India, Indonesia, Taiwan, Vietnam.

Xanthoparmelia (Vain.) Hale; Parmeliaceae

Xanthoparmelia congensis (Stein) Hale, Phytologia 28 : 486 (1974).

Thailand. Chiang Mai (Doi Saget), Loei (Ban Sui, Phu Kradung), Lumpang (Khuntan N.P.), Phitsanulok (Lan Hin Taek, Phu Hin Rongkla, N.P.), Sakonnakhon (Phu Pan N.P.). Pooprang et al. (1999: 125), Moon et al. (2000a: 105).

SPECIMENS. RAMK, TNS.

ASIA. China (incl. Hong Kong).

Xanthoparmelia mougeotina (Nyl.) D.J. Galloway, New Zealand J. Bot. 18: 538 (1981).

THAILAND. Lumpang (Khuntan N.P.). Pooprang et al. (1999: 125). SPECIMEN. RAMK.

ASIA. Papua New Guinea.

ACKNOWLEDGements. We are grateful to those organisations and individuals who have assisted this project over many years: the Leverhulme Trust who provided the initial funding to Pat Wolseley and Begoña AguirreHudson for this research in Thailand; The Natural History Museum for supporting this research during and since initial funding; Prof. Teuvo Ahti (Helsinki), Prof. Jack Elix (Canberra), Dr Seppo Huhtinen (Turku), Dr Hiroyuki Kashiwadani (Tokyo), Laszlo Lökös (Budapest), Dr Simone Louwhoff (London), the Royal Forestry Department, Thailand; Prof. Kansri Boonpragob, Dr Natsurang Homchantara and the staff and students of the Lichen Research Group, Ramkhamhaeng University, Bangkok; Dr Wanaruk Saipunkaew (Chiang Mai University), Dr Oy Kanjanavanit and M.R. Smansnid Svasti.

This catalogue is dedicated to Thai lichenologists and especially to Prof. Kansri Boonpragob and Dr Natsurang Homchantara under whose direction lichenology now flourishes in Thailand. 


\section{REFERENCES}

Abbayes, H. des 1956. Quelques Cladonia (lichens) des regions inter-tropicales, nouveaux ou peu connus, conservés dans l'herbier de Kew. Kew Bulletin 2: 259-266.

1964 ['1963']. Lichens nouveaux ou interessants du Vietnam. Revue Bryologique et Lichénologique 32: 216-222.

Aguirre-Hudson, 13. \& Wolseley, P.A. 1994. Bibliography of southeast Asian lichenology, 1810-1992. Journal of the Hattori Botanical Laboratory 76: 313-350. Ahti, T. 1984. The status of Cladina as a segregated from Cladonia. Beiträge zur Nova Hedwigia 79: 25-61.

Archer, A.W. 1997. The lichen gcnus Pertusaria in Australia. Bibliotheca Lichenologica 69: $1-249$.

Arvidsson, L. 1982. A monograph of the lichen genus Coccocarpia. Opera Botanica 67: $1-99$.

Asahina, Y. 1968. Lichenologische Notizen (211-212). Joumal of Japanese Botany 43: $495-499$.

1969. Lichenologische Notizen (215-216). Journal of Japanese Botany 44: 33 39.

Awasthi, D.D. 1960. On a collection of macrolichens by the Indian expedition to Chooyu, east Nepal. Proceedings of the Indian Academy of Sciences 51: 169-180.

1975. A monograph of the lichen genus Dirinaria. Bibliotheca Lichenologica 2: $1-108$.

- 1977. ['1976'] Lichen genus Parmelia in India I - subgenera Parmelia and Ampligymuia. Biological Memoirs, Lich. I 1(1-2): 155-229.

1986. Lichen genus Usuea in India. Journal of the Hattori Botanical Laboratory 61: $333-421$.

Boonpragob, K., Homchantara, N., Coppins, B.J., McCarthy, P.M. \& Wolseley, P.A. 1998. An introduction to the lichen flora of Khao Yai National Park, Thailand. Botanical Journal of Scotland 50(2): 209-219.

Culberson, C.F., Culberson, W.L. \& Johnson, A. 1981. A standarized TLC analysis of B-orcinol depsidones. The Bryologist 84: 16-29.

\& Hale, M.E. 1973. Chemical and morphological evolution in Parmelia sect. Hypotrachyna: product of ancient hybridization? Brittonia 25: 162-173.

David, F., Elix, J.A. \& Samsudin, M.W. 1990. Two new aliphatic acids from the lichen Parmotrema praesorediosum. Australian Journal of Chemistry 43: 1297-1300.

Degelius, G. 1974. The lichen genus Collema with special reference to the extraEuropean species. Symbolae Botanicae Upsalienses 20(2): 1-215.

Elix. J.A. 1996. A revision of the lichen genus Relicina. Bibliotheca Lichenologica 62:1-150.

Hafellner, J. 1981. Monographie der Flechtengattung Letrouitia (Lecanorales, Teloschistinae). Nova Hedwigia 35: 645-729.

- 1993. Die Gattung Pyrrhospora in Europa. Eine erste Übersicht mit einem Bestimmungsschlüssel der Arten nebst Bemerkungen zu einigen aussereuropäischen Taxa (lichenisierte Ascomycotina, Lecanorales). Herzogia 9: 725-747.

Hale, M.E. 1965a. A monograph of Parmelia subgenus Amphigymia. Contribution. of the United States National Herbarium 36(5): 193-358.

1965b. Six new species of Parmelia from southeast Asia. Journal of Japanese Botany 40: 199-205.

1968. New Parmeliaceae from southeast Asia. Journal of Japanese Botany 43: 324-328.

1971. Morden-Smithsonian expedition to Dominica: the lichens (Parmeliaceae). Smithsonian Contributions to Botany 4: 1-25

1975. A monograph of the lichen genus Relicina (Parmeliaceae). Smithsonian Contributions to Botany 26: 1-32.

- 1976a. A monograph of the lichen genus Bulbothrix Hale (Parmeliaceae). Snithsonian Contributions to Botany 32: 1-29.

1976b. A monograph of the lichen genus Pseudoparmelia Lynge (Parmeliaceae). Smithsonian Contributions to Botany 31: 1-62.

- 1976c. A monograph of the lichen genus Parnelina Hale (Parmeliaceae). Smitlsonian Contributions to Botany 33: $1-60$.

1981. A revision of the lichen family Thelotremataceae in Sri Lanka, Bulletin of the British Museum (Natural History), Botany 8: 227-332.

_ \& Kurokawa, S. 1964. Studies on Parmelia subgenus Parmelia. Contributions from the U.S. National Herbarium 36(4): 121-191.

Hamalainen M. \& Pinratana A. 2001. Allas of the dragonflies of Thailand. Bangkok. Harris, R.C. 1993 ['1991']. A revision of Polymeridium (Müll. Arg.) R.C. Harris (Trypetheliaccae). Boletim do Museu Paraense Emílio Goeldi, Série Botānica 7 : 619-644.

1998. A preliminary revision of Pseudopyrenula Müll. Arg. (lichenized Ascomycetes, Trypetheliaceae) with a redisposition of the names previously assigned to the genus. In M.G. Glenn, R.C. Harris, R. Dirig \& M.S. Cole (Eds) Lichenographia Thomsoniana: North Anerican liclenology in honour of John W. Thomson, pp. 133 148. New York.

Hawksworth, D.L. 1985. A redisposition of the species referred to the ascomycete genus Microthelia. Bulletin of the British Museum (Natural History), Botany 14: 43-181

- Kirk, P.M., Sutton, B.C. \& Pegler, D.N. 1995. Ainsworth \& Bisby's Dictionary of the Fungi. 8th ed. Wallingford.
\& Yoshimura, 1. 1973. 358. Proposal to conserve the generic name Gymnoderma Nyl. 1863. [Lichenes] against Gymnoderna Humb ex Steud, 1824. [Fungi]. Taxon 22: 503.

1lenssen, A. 1963. Eine revision der Flechtenfamilien Lichinaceae und Ephebaceae Symbolae Botanicae Upsalienses 18: 1-123.

1979. Problematik der Gattungsbegrenzung bei den Lichinaceen. Berichte der Deutschen Botanischen Gesellschaft 92: 483-506.

1989. Metamelanea and Stromatella, new genera of Lichinaceae. Lichenologist 21: $101-118$.

Herre, A.W.T.C. 1963. The lichen genus Usnea and its species at present known from the Philippines. Philippine Journal of Science 92(1); 41-76.

Holmgren, P.K., Holmgren, N.H. \& Barnett, L.C. 1990. Index Herbariorum Part 1: The Herbaria of the World. New York.

Homchantara, N. 1999. The taxonomic and ecological aspects of the Thelotremataceae in southeast Asia. Unpublished Ph.D. thesis. John Moores University Liverpool.

\& Coppins, B.J. (2002). New species of the lichen family Thelotremataceae in SE Asia. Lichenologist 34: (in press).

Huovinen, K. \& Ahti, T. 1986. The composition and contents of aromatic lichen substances in Cladonia, section Unciales. Annales Botanici Fennici 23: 173-188.

Jorgensen, P.M. 1997. Further notes on hairy Leptogium species. Symbolae Botanicae Upsalienses 32(1): 113-130.

2000. Notes on some east-Asian species of the lichen genus Fuscopannaria. Journal of the Hattori Botanical Laboratory 89: 247-259.

- 2001. New species and records of the lichen family Pannariaceae from Australia. Bibliotheca Lichenologica 78: 109-139

Kalb, K. \& Elix, J.A. 1995. The lichen genus Physcidia. Bibliotheca Lichenologica 57: 265-296.

Kashiwadani, H. 1975. Ennumeration of Anaptychiae and Parmeliae of Papua New Guinea. Reports of the Cryptogams in Papua New Guinea: 75-85. Tokyo.

- 1977. Rare lichens collected in the 1zu Islands. Memoirs of the National Science Museum, Tokyo, Botany 10: 43-48.

1988. Ramalina hossei Vainio (lichen) found in Buthan and Formosa. Bulletin of the National Science Museum, Tokyo, Botany 14: 129-133.

— \& Gradstein,S.R. 1982. Notes on Baeomyces sanguineus Asahina and Gymnoderma coccocarpum Nyl. Miscellanea Bryologica et Lichenologica 9: 79-81.

Kurokawa, S. 1959. Anaptychiae (lichens) and their allies in Japan (2). Journal of Japanese Botany 34: 174-184.

1961. Anaptychiae (lichens) and their allies of Japan (6). Journal of Japanese Botany 36: 51-56.

1962. A monograph of the genus Anaptychia. Beihefte zur Nova Hedwigia 6: 1 155.

1973. Supplementary notes on the genus Anaptychia. Journal of the Hattori Botanical Laboralory 37: 563-607.

- 1980. Cetrariopsis, a new genus in the Parmeliaceae, and its distribution. Memoirs of the National Science Museum, Tokyo 13: 139-142.

1988. Materials for the distribution of lichens in Japan (10). Journal of Japanese Botany 63: 167-168.

- 1991. Rimeliella, a new lichen genus related to Rimelia of the Parmeliaceae. Annals of the Tsukuba Botanical Garden 10: 1-14.

1998. A new record and a new species in Myelochroa (Parmeliaceae). Journal of Japanese Botany 73: 113.

— \& Kashiwadani, H. 1988. Indices to taxa distributed under Lichens rariores et critici exsiccati Fascicules I-XIV (Nos. I-700). Tokyo.

Lumbsch, H.T., Guderley, R. \& Elix, J.A. 1996. A revision of some species in Lecanora sensu stricto with a dark hypothecium (Lecanorales, Ascomycotina). The Bryologist 99: 269-291,

McCarthy, P.M. 1995. A reappraisal of Clathroporina Müll. Arg. (Trichotheliaceae). Lichenologist 27: 321-350.

1999. Three new species of Porina (Trichotheliaceae) from Thailand. Lichenologist 31: $239-246$.

Moon, K.H., Kurokawa, S. \& Kashiwadani, H. 2000a. A list of Thailand species of Parmelia (sens. lat.) Preserved in the National Science Museum. In K. Matsura (Ed.) Proceedings of the First and Second Symposia on Collection Building and Natural History Studies in Asia. National Science Museum Monographs 18. Tokyo: 97-106.

,$--\&-2000 \mathrm{~b}$. A new species of Hypotrachyna (lichenised Ascomycetes, Parmeliaceae) from Thailand. Bulletin of the National Science Museum, Tokyo, ser. B, 26: 135-138.

Mongkonsuk, P., Boonpragob, K., Homchantara, N. \& Boonchai, W. 1996. Diversity of the lichens at Queen Sirikit Botanic Garden, Mae Rim, Chiang Mai. Botanical Garden Organization Newsletter 4: 13-22.

Motyka, J. 1936. Lichenum generis Usnea studium monographicum, Pars systematica I: 1-304. Lublin.

- 1937-1938. Lichenes generis Usnea studium mongraphicum, Pars systematica 2: 305-651. Lublin.

Oshio, M. 1976. Pertusaria variolosa (Kremp.) Vain. and allied species. Miscellanea Bryologica et Lichenologica 7(6): 114-116.

Parniasto, E. 1978. The genus Dyctionema (Thelophorolichenes). Nova Hedwigia 29: 99-144 
Patwardhan, P.G. \& Kulkarni, C.R. 1976. Some additions to the lichen flora of India IV: Graphis and Graphina (Family Graphidaceae). Biovigyanam 2: 123-132.

- 1977a. A contribution to our knowledge of the lichen flora of 1ndia 1: Family Thelotremataceae. Kavaka 5: 1-17.

1977b. Some additions to the lichen flora of India V: Genera Phaeographis and Phaeographina (Family Graphidaceae). Current Science 46: 720-721.

\& Prabhu, A.V. 1977. Some additions to the lichen flora of India 11: Parmelina Hale and Parmotrema Mass. Biovigyanam 3: 111-113.

Paulson, R. 1930. Lichens from Kaw Tao, an island in the Gulf of Siam. The Journal of the Siam Society, Natural History Supplement 8(2): 99-101.

Pooprang, T., Boonpragob, K. \& Elix, J.A. 1999. New species and new records in the lichen family Parmeliaceae (Ascomycotina) from Thailand. Mycotaxon 71: 111127.

Rogers, R.W. 1986. The genus Pyxine (Physciaceae, lichenized Ascomycetes) in Australia. Australian Journal of Botany 34: 121-154.

\& Stevens, G.N. 1988. Usnea baileyi complex (Parmeliaceae, Lichenised Ascomycetes) in Australia. Australian Systematic Botany 1: 355-361.

Rostrup, F.G.E. 1902. Fungi. In J. Schmidt (Ed.), Flora of Koh Chang; Contributions to the knowledge of the vegetation in the Gulf of Siam. Botanisk Tidsskrift Kjobenhavn 24: $355-367$

Salisbury, G. 1971. The Thelotrenıata of Angola and Mocambique. Revista de Biologia 7: $271-280$.

Santesson, R. 1952. Foliicolous lichens I: A revision of the taxonomy of the obligately foliicolous lichenized fungi. Symbolae Botanicae Upsaliensis 12(1): 1-590.

Santisuk, T. 1988. An account of the vegetation of northern Thailand. Geoecological Research 5: 1-101.

Sato, M. 1938. Enumeratio Lichenum Ins. Formosae (IV). Journal of Japanese Botany 14: $463-469$.

- 1962. Lichenes of Doi Inthanon and Doi Suthep northwestern Thailand. In T. Kira \& T. Umeaso (Eds), Nature and life in Southeast Asia I1: 1-4.

Sérusiaux, E. 1985. Goniocysts, goniocystangia and Opegrapha lambinonii and related species. Lichenologist 17: 1-25.

Sethy, P.K., Nagarkar, M.B. \& Patwardhan, P.G. 1987. Materials for a lichen flora of the Andaman lslands - 111. Mycotaxon 28: 191-198.

Singh, K.P. 1979. Some new records of lichens from India. Bulletin of the Botanical Survey of India 21: 221-223.

Sipman, H.J.M. 1983. A monograph of the lichen family Megalosporaceae. Bibliotheca lichenologica 18: 1-24l.

Staiger, B. \& Kalb, K. 1995. Haematomma-studien. 1. Die Flechtengattung Haematomma. - Bibliotheca Lichenologica, 59, Berlin.

Stenroos, S. 1986. The family Cladoniaceae in Melanesia 1. Cladonia sect. Uncialis. Annales Botanici Fennici 23: 161-164.

1988. The family Cladoniaceae in Melanesia 4. The genera Cladia, Cladina, Calathaspis and Thysanothecium. Annales Botanici Fennici 25: 207-218.
Stevens, G.N. 1990. Usnea himantodes Stirton and its synonyms. Lichenologist 22: $409-412$.

Thor, G. 1990. The lichen genus Chiodecton and five allied genera. Opera Botanica 103: $1-92$.

Vainio, E.A. 1907. Lichenes novi rarioresque, 4. Hedwigia 46: 168-181.

- 1909. Lichenes. In J. Schmidt (Ed.), Flora of Koh Chang; Contributions to the knowledge of the vegetation in the Gulf of Siam. Botanisk Tidsskrift Kjobenhavn 29: $104-151$.

- 1921. Lichenes in summo monte Doi Sutep (circ. 1675 m.s.m.) in Siam boreali anno 1904 AD: re C.C. Hosseus collect. Suomalise Elain-ja Kasvitieteelisen Seuran Vanamon Julkaisuja 1(3): 33-55.

Vongshewarat, K., McCarthy, P. M., Mongkolsuk, P. \& Boonpragob, K. 1999. Additions to the lichen flora of Thailand. Mycotaxon 70: 227-236.

Wirth, M. \& Hale, M.E. 1978. Morden-Smithsonian expedition to Dominica: The lichens (Graphidaceae). Smithsonian Contributions to Botany 40: 1-64.

Wolseley, P. 1991. Observations on the composition and distribution of the 'Lobarion' in forests of south east Asia. In D.J. Galloway (Ed.), Tropical lichens: their systematics, conservation, and ecology. Systematics Association Special Volume $\mathbf{4 3}$ 217-243. Oxford

\& Aguirre-Hudson, B. 1991. Lichens as indicators of environmental change in the tropical forests of Thailand. Global Ecology and Biogeography Letters 1: 170175.

- \& - 1995. Key to lichen genera in Thailand with special reference to epiphytic taxa, Part 1: Macrolichens. Natural History Bulletin of the Siam Society 43: 303 335.

- $\&$ 1997a. The ecology and distribution of lichens in tropical deciduous and evergreen forests in Thailand. Journal of Biogeography 24: 327-343.

$-\boldsymbol{\&}-1997 \mathrm{~b}$. Fire in tropical dry forests: corticolous lichens as indicators of recent ecological changes in Thailand. Journal of Biogeography 24: 345-362.

— Moncrieff, C. \& Aguirre-Hudson, B. 1994. Lichens as indicators of environmental stability and change in the tropical forests of northern Thailand. Global Ecology and Biogeography Letters 4: 116-123.

Yoshimura, I. 1971. The genus Lobaria of eastern Asia. Journal of the Hattori Botanical Laboratory 34: 231-364.

- 1978. Some lichens of Thailand collected by the Danish botanists, 1958-68. Bulletin of the Kochi Gakuen Junior College 9: 35-40.

- 1982. Distribution of Gyminoderma coccocarpum Nyl. and G. insulare Yoshim. \& Sharp. Bulletin of the Kochi Gakuen Junior College 13: 83-86.

\& Sharp, A.J. 1968. A revision of the genus Gymnoderma. American Journal of Botany 55: 635-640.

Zahlbruckner, A. 1926. Lichenes (Flechten). In A. Engler \& K. Prantl (Eds), Die Naturlichen Pflanzenfamilien 8: 1-270. Leipzig.

1933. Flechten der Insel Formosa 1I. Feddes Repertorium Specierum Novarum Regni Vegetabilis 33: 22-68. 



\section{Botany Series}

Earlier Botany Bulletins are still in print. The following can be ordered from lntercept (address on inside front cover). Where the complete backlist is not shown, this may also be obtained from the same address.

Volume 24

No. 1 Pre-Linnaean references for the Macaronesian flora found in Leonard Plukenet's works and collections. J. Francisco-Ortega, A. Santos-Guerra and C.E. Jarvis. 1994. Pp. 1-34, 16 figs. Studies on the lichen genus Sticta (Schreber) Ach.: II. Typification of taxa from Swartz's Prodromus of 1788. D.J. Galloway. 1993. Pp. 35-48, 9 figs.

Seaweeds of the western coast of tropical Africa and adjacent islands: a critical assessment. IV. Rhodophyta (Florideae) 4. Genera L-O. D.M. John, G.W. Lawson, J.H. Price, W.F. Prud'homme van Reine and W.J. Woelkerling. 1994. Pp. 49-90, 1 fig.

Studies on the Cretan flora 3. Additions to the flora of Karpathos. N.J. Turland and L. Chilton. 1994. Pp. 91-100, 1 fig. $£ 43.25$

No. 2 Observations on the benthic marine algal flora of South Georgia: a floristic and ecological analysis. D.M. John, P.J.A. Pugh and I. Tittley. 1994. Pp. 101-114, 8 figs.

Studies in Pseudocyphellaria (Lichens) IV. Palaeotropical species (excluding Australia). D.J. Galloway. 1994. Pp. 115160,36 figs.

Morphology and ecology of seedlings, fruits and seeds of Panama: Bixaceae and Cochlospermaceae. N.C. Garwood. 1994. Pp. 161-172, 2 figs.

A study of Bixa (Bixaceae), with particular reference to the leaf undersurface indumentum as a diagnostic character. R.E. Dempsey and N.C. Garwood. 1994. Pp. 173-180, 2 figs.

\section{Volume 25}

No. 1 A revision of Rutilaria Greville (Bacillariophyta). R. Ross. 1995. Pp. 1-94, 76 figs, 20 plates.

William Roxburgh's St Helena plants. Q.C.B. Cronk. 1995. Pp. 95-98.

No. 2 Seaweeds of the western coast of tropical Africa and adjacent islands: a critical assessment. IV. Rhodophyta (Florideae) 5. Genera P. G.W. Lawson, W.J. Woelkerling, J.H. Price, W.F. Prud'homme Van Reine and D.M. John. 1995. Pp. 99-122, 1 fig. A new species of Odontorrhynchos (Orchidaceae, Spiranthinae) from Boliva. D.L. Szlachetko. 1995. Pp. 123125,1 fig.

Linnaeus's interpretation of Prospero Alpino's De plantis exoticis, with special emphasis on the flora of Crete. N.J. Turland. 1995. Pp. 127-159, 27 figs.

Book review. M.G. Gilbert. 1995. P. 161.

Volume 26

No. 1 A morphological study of Chaetoceros species

(Bacillariophyta) from the plankton of the Pacific ocean of Mexico. D.U. Hernández-Becerril. 1996. Pp. 1-73, 52 plates.

$£ 43.40$

No. 2 Studies in the genus Hypericum L. (Guttiferae) 6. Sections 20. Myriandra to 28. Elodes. N.K.B. Robson. 1996. Pp. 75-217, 43 maps, 29 figs.

$£ 43.40$

Volume 27

No. 1 Notes on the diatom species Tetracyclus castellum (Ehrenb.) Grunow with a description of Tetracyclus pseudocastellum nov. sp. D.M. Williams. 1997. Pp. 1-5, 8 figs.

A new species of Calymperes (Musci: Calymperaceae) from Peninsular Malaysia. L.T. Ellis. 1997. Pp. 7-9, 1 fig. A phylogenetic conspectus of the tribe Hyoscyameae (Solanaceae). A.L. Hoare and S. Knapp. 1997. Pp. 11-29. 7 figs. A revision of Solanum section Pteroidea: Solanaceae. S. Knapp and T. Helgason. 1997. Pp. 31-73, 23 figs.

$£ 43.40$

No. 2 Systematics of Pogostemon (Labiatae) G.R. Bhatti and M.
Volume 28

lngrouille. 1997. Pp. 77-147, 40 figs.

$\$ 43.40$

No. 1 Morphology and ecology of seedlings, fruits and seeds of Panamá: Vochysiaceae. N.C. Garwood. 1998. Pp. 1-16, 3 figs. A revision of the genus Mandragora (Solanaceae). S. Ungricht, S. Knapp and J.R. Press. 1998. Pp. 17-40, 9 figs.

The pteridophytes of São Tomé and Príncipe (Gulf of Guinea).

E. Figueiredo. 1998. Pp. 41-66, 2 figs.

$£ 43.40$

No. 2 A revision of Brillantaisia (Acanthaceae). K. Sidwell. 1998.

Pp. 67-113, 5 maps, 16 figs.

Seaweeds of the western coast of tropical Africa and adjacent islands: a critical assessment. 1V. Rhodophyta (Florideae) 6.

Genera [Q] R-Z, and an update of current names for nongeniculate Corallinales. W.J. Woelkerling, G.W. Lawson, J.H. Price, D.M. John and W.F. Prud'homme van Reine. 1998. Pp. 115-150, 1 fig.

$£ 43.40$

Volume 29

No. 1 The moss family Calymperaceae (Musci) in the Philippines.

L.T. Ellis. 1999. Pp. 1-46, 25 figs.

Revision of Hibiscus section Furcaria (Malvaceae) in Africa and Asia. F.D. Wilson. 1999. Pp. 47-79, 6 figs.

No. 2 Catalogue of the holdings in The Natural History Museum (London) of the Australian botanical drawings of Ferdinand Bauer (1760-1826) and cognate materials relating to the Investigator voyage of 1801-1805. D.J. Mabberley and D.T.

Volume 30 Moore. 1999. Pp. 81-226, 268 figs.

$\mathfrak{\$ 4 3 . 4 0}$

No. 1 A new species of Heisteria (Olacaceae) from Mesoamerica. Q. Jiménez and S. Knapp. 2000. Pp. 1-6.

Three new species of Pilea (Urticaceae) from Costa Rica and Panama. A.K. Monro. 2000. Pp. 7-12.

A revision of Solanum thelopodium species group (section Anthoresis sensu Seithe, pro parte): Solanaceae. S. Knapp. 2000. Pp. 13-30.

No. 2 The genus Polystichum (Dryopteridaceae) in Africa. J.P. Roux. 2000. Pp. 33-79.

Recent records of pteridophytes for Belize, Central America. D.A. Sutton, A. Hughes and B. Bulmer-Thomas. 2000. Pp. 8199.

Collections of flowering plants by Francis Buchanan-Hamilton from Nepal, 1802-1803. J.R. Press and K.K. Shrestha. 2000.

Volume 31 Pp. 101-130.

$£ 43.40$

No. 1 New synonymy in some Asian species of Syrrhopodon (Calymperaceae: Musci). L.T. Ellis. 2001. Pp. 1-4, 2 figs. Two new species of Pilea (Urticaceae) from Panama.

A.K. Monro. 2001. Pp. 5-7, 1 fig.

Synopsis of Mesoamerican Pilea (Urticaceae), including eighteen typifications and a key to the species. A.K. Monro. 2001. Pp. 9-25.

The Japanese plant collection of Engelbert Kaempfer (16511716) in the Sir Hans Sloane Herbarium at The Natural History Museum, London. P-A. Hinz. 2001. Pp. 27-34, 1 fig.

Index to new taxa. P. 35.

$£ 43.40$

No. 2 Studies in the genus Hypericum L. (Guttiferae) 4(1). Sections 7. Roscyna to 9. Hypericum sensu lato (part 1). N.K.B. Robson. 2001. Pp. 37-88.

A bibliography of the scientific work of Boris V. Skvortzov (1896-1980) with commentary on the publications concerning diatoms (Bacillariophyta). D.M. Williams and G. Reid. 2001. Pp. 89-106.

On the identity of Pleurosigma angulatum (Bacillariophyta) and related species. G. Reid.2001. Pp. 107-117. Index to new taxa. P. 119. 


\section{CONTENTS}

1 Taxonomic notes on some African species in the family Calymperaceae (Musci) L.T. Ellis

7 Typification of Ranunculus bullatus L. (Ranunculaceae)

. R.M. Baldini and C.E. Jarvis

13 Catalogue of the lichens of Thailand

P.A. Wolseley, B. Aguirre-Hudson and P.M. McCarthy

\section{CAMBRIDGE UNIVERSITY PRESS}

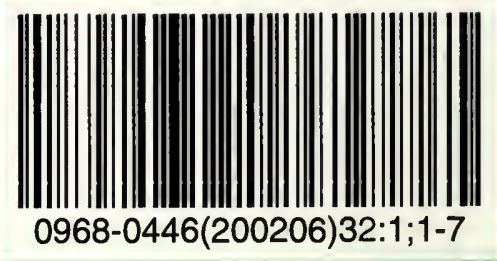

Bulletin of The Natural History Museum

\section{BOTANY SERIES}

Vol. 32, No. 1, June 2002 